\title{
Investigation of fabrication process development for integrated optical grating structures
}

\author{
Divya Pisharoty \\ West Virginia University
}

Follow this and additional works at: https://researchrepository.wvu.edu/etd

\section{Recommended Citation}

Pisharoty, Divya, "Investigation of fabrication process development for integrated optical grating structures" (2005). Graduate Theses, Dissertations, and Problem Reports. 1614.

https://researchrepository.wvu.edu/etd/1614

This Thesis is protected by copyright and/or related rights. It has been brought to you by the The Research Repository @ WVU with permission from the rights-holder(s). You are free to use this Thesis in any way that is permitted by the copyright and related rights legislation that applies to your use. For other uses you must obtain permission from the rights-holder(s) directly, unless additional rights are indicated by a Creative Commons license in the record and/ or on the work itself. This Thesis has been accepted for inclusion in WVU Graduate Theses, Dissertations, and Problem Reports collection by an authorized administrator of The Research Repository @ WVU. For more information, please contact researchrepository@mail.wvu.edu. 


\title{
Investigation of Fabrication Process Development for Integrated Optical Grating Structures
}

\author{
Divya Pisharoty \\ Thesis submitted to the \\ College of Engineering and Mineral Resources at \\ West Virginia University \\ in partial fulfillment of the requirements \\ for the degree of
}

MASTER OF SCIENCE

in

ELECTRICAL ENGINEERING

Dr. Lawrence Hornak, Ph.D., Chair

Dr. Dimitris Korakakis, Ph.D.

Dr. Aaron Timperman, Ph.D.

Lane Department of Computer Science and Electrical Engineering

Morgantown, West Virginia

2005

Keywords: Diffraction, E-beam lithography, Reactive Ion Etch, Laser Ablation

(C) Copyright 2005 Divya Pisharoty 


\section{Abstract \\ Investigation of Fabrication Process Development for Integrated Optical Grating Structures}

Divya Pisharoty

Sub-micron optical gratings are essential integrated elements to couple light into waveguides and for optical monitoring of microfluidic channels or MEMS devices. Since the required features in the sub-micron regime are difficult to achieve by mechanical ruling and photolithography, alternate fabrication techniques are investigated. Exposure by e-beam and laser are two direct write methods which are used. These methods preclude the need of a mask, require few processing steps, and can be used to write features of sizes desired for optical gratings. In this research, we explore and develop processes to create sub-micron gratings by both these methods. A resist bi-layer is patterned by e-beam exposure. After metal lift-off, the grating structure is transferred into silicon by reactive ion etching (RIE). Rectangular gratings with 200nm-500nm feature size and 50\% pitch were successfully fabricated. Borofloat, silica and alumina have been etched using chrome as a mask with vertical sidewalls. Grating structures of any pitch can be created in these materials using e-beam writing in resist and RIE. A $255 \mathrm{~nm}$ copper vapor laser was used to ablate lines in borosilicate glass. 


\section{Acknowledgements}

Dr. Hornak, my advisor, has always been a source of inspiration during my graduate program. I would like to thank him for his patience and support throughout the research. I would also like to thank my other committee members Dr. Dimitris Korakakis and Dr. Aaron Timperman, for their insight and support. I thank Dr. Kolin Brown for his assistance and support in the microfabrication lab, James Lenke and Trust Razunguzwa for training me to use the Copper Vapor Laser, Dr. David Letterman, Hongtao Shi and Debin Li for the use of the direct-write SEM and Liviu Magean and Steve Carpenter for helping me with the SEM images.

Additionally, I would like to thank my colleagues of the research group, especially Timothy Cornell for e-beam evaporation of chrome, and Chris Miller, Deepa Konduparthi and Chris Hollandsworth for assisting in optical characterization. I also thank my previous colleagues Scott Rittenhouse, William McCormick, and Shantanu Pathak who helped me in the lab when I had just started my research.

In addition, I would like to thank my parents for encouraging me to pursue a graduate degree and for their patience as I worked my way through it, my sister for her love and my friends for their constant support and encouragement. 


\section{Contents}

$\begin{array}{ll}\text { Abstract } & \text { ii }\end{array}$

Acknowledgements $\quad$ iii

Contents $\quad$ iv

List of Figures

List of Tables $\quad x$

1 Introduction 1

1.1 Background and Applications . . . . . . . . . . . . . . . . . . 1

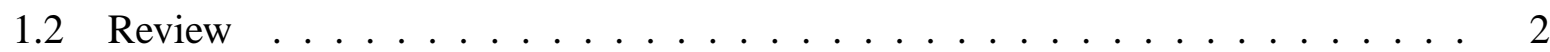

1.2.1 Methods of Manufacture . . . . . . . . . . . . . . . . 2

1.2.2 Grating Types . . . . . . . . . . . . . . . . . 3

1.3 Research . . . . . . . . . . . . . . . . . . . 3

1.4 Organization of Thesis $\ldots \ldots \ldots \ldots \ldots$

2 Diffraction and Fabrication Methods $\quad 6$

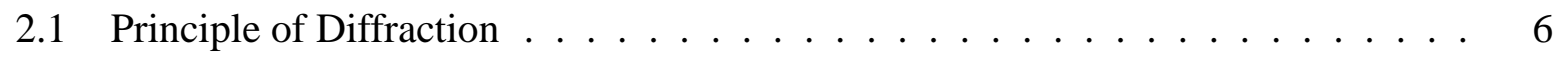

2.2 Grating equation $\ldots \ldots \ldots \ldots \ldots \ldots$

2.3 Fabrication Methods . . . . . . . . . . . . . . . . 11

2.3.1 Mechanical ruling ........................ 11

2.3.2 Photolithography . . . . . . . . . . . . . . . . . 11

2.3.3 Electron beam lithography $(\mathrm{EBL}) \ldots \ldots \ldots$

2.3.4 Laser Ablation . . . . . . . . . . . . . . . . . . . . . 17 
2.3.5 Interferometric Fabrication . . . . . . . . . . . . . . . 18

3 Research Fabrication Methods $\quad 23$

3.1 Choice of Substrate . . . . . . . . . . . . . . . . . . . 23

3.2 Direct-write and RIE . . . . . . . . . . . . . . . . . . . . . . . 24

3.2 .1 Grating depth . . . . . . . . . . . . . . . . 24

3.2.2 Creating gratings by direct write and reactive ion etching . . . . . . . 24

3.3 Laser ablation and phase mask . . . . . . . . . . . . . . . 37

3.3 .1 Grating period ...................... 40

3.3.2 Laser Process . . . . . . . . . . . . . . . . . . . . 40

4 Fabrication Process Development and Results 43

4.1 Direct write . . . . . . . . . . . . . . . . . . . 43

$4.1 .1 \quad$ PMMA495 A6 . . . . . . . . . . . . . . . . 43

4.1 .2 Direct write on PMMA 495 A2 . . . . . . . . . . . 51

4.1.3 Direct write on PMMA 495 A2 and copolymer EL6 . . . . . . . . 53

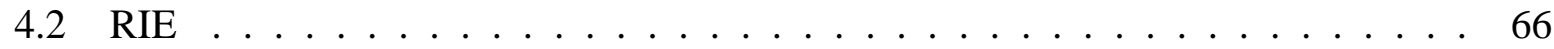

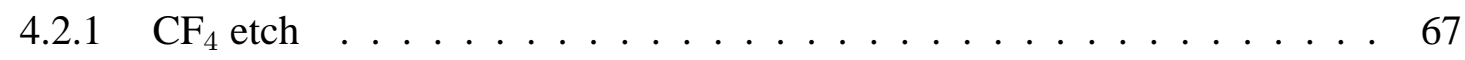

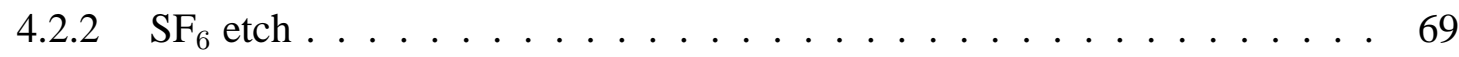

4.3 Direct write and RIE . . . . . . . . . . . . . . . . 83

4.4 Laser Ablation $\ldots \ldots \ldots$. . . . . . . . . . . . . . . . . . . . . . 84

5 Optical Testing and Characterization $\quad 88$

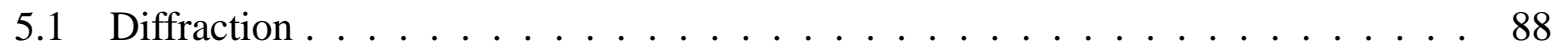

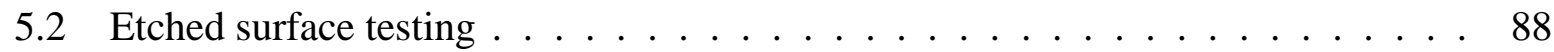

5.3 Optical Characterization $\ldots \ldots \ldots \ldots \ldots$. . . . . . . . . . . . 92

5.3 .1 Intensity as a function of wavelength $\ldots \ldots . \ldots . \ldots 92$

5.3.2 Intensity as diffraction angle is varied . . . . . . . . . . . 92

5.3 .3 Set-up to reduce spot-size for 50 micron gratings $\ldots \ldots$. . . . . . . 95

6 Conclusions and Future Work 96 


\section{List of Figures}

1.1 Possible locations of gratings to couple light into the Alumina waveguide . . . 2

1.2 Research Process Development . . . . . . . . . . . . . . . . . . 4

2.1 Double-slit Diffraction ${ }^{1} \ldots \ldots \ldots \ldots \ldots$

2.2 Phase Angle Relation in a Grating $2 \ldots \ldots$. . . . . . . . . . . . . 7

2.3 Phase Angle Relation in a Transmission Grating ${ }^{3} \ldots \ldots$. . . . . . . . . . 8

2.4 Diffraction of white light by a Lamellar Grating . . . . . . . . . . . . . . . . . 9

2.5 Phase mask to generate interference pattern $\ldots \ldots \ldots \ldots$

2.6 Fiber Bragg Grating ${ }^{5} \ldots \ldots \ldots \ldots \ldots \ldots$

2.7 Photolithographic processing and etching $\ldots \ldots \ldots \ldots \ldots$

2.8 PMMA monomer . . . . . . . . . . . . . . . . . . . . . 13

2.9 Monte-Carlo simulation for $20 \mathrm{KV}[1] \ldots \ldots \ldots \ldots$

2.10 Recording FBG in $+1 /-1 \operatorname{mode}^{7} \ldots \ldots \ldots \ldots \ldots$

2.11 Phase mask in $+1 /-1$ mode $\ldots \ldots \ldots \ldots \ldots \ldots$

2.12 Phase mask in $0 /-1$ mode . . . . . . . . . . . . . . . . . . . . 21

3.1 Chemical properties of borofloat ${ }^{1} \ldots \ldots \ldots \ldots \ldots$

3.2 Process steps of fabrication of gratings by e-beam and RIE . . . . . . . . . . . 24

3.3 Film thickness of PMMA 495 A6 and A2 at different spin speeds ${ }^{2} \ldots \ldots$

3.4 Copolymer resists at different spin speeds ${ }^{2} \ldots \ldots \ldots \ldots \ldots$

3.5 Leica E-beam system ${ }^{4} \ldots \ldots \ldots \ldots \ldots \ldots \ldots \ldots$

3.6 Chrome as mask for plasma etch . . . . . . . . . . . . . 30

3.7 Metal deposition on single PMMA layer . . . . . . . . . . . . . . . . 32 
3.8 Idealized lift-off profile . . . . . . . . . . . . . . . . 32

3.9 Metal deposition on bilayer . . . . . . . . . . . . . . . 32

3.10 E-beam interaction in bilayer . . . . . . . . . . . . . . 33

3.11 Copolymer-resist bilayer lift-off process . . . . . . . . . . . . . 33

3.12 Schematic of laser system ${ }^{9} \ldots \ldots \ldots \ldots \ldots$

3.13 Reduced height of lines due to promixity . . . . . . . . . . . . . . 40

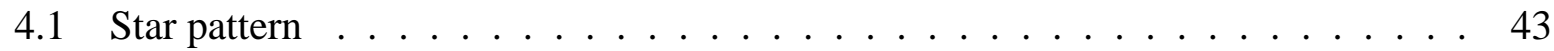

4.2 Rotation positions at $\mathrm{X}=580, \mathrm{Y}=333 \ldots \ldots \ldots \ldots \ldots$

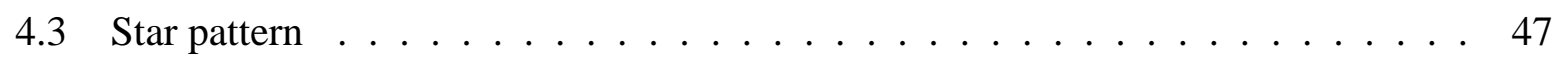

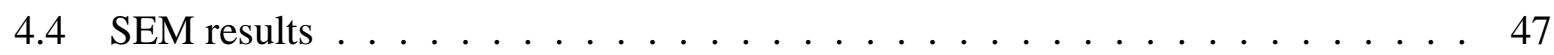

$4.5120 \mu \mathrm{C} / \mathrm{cm}^{2}$ is over-exposure for all line sizes . . . . . . . . . . . . . . . 49

4.6 SEM results for exposure of $80 \mu \mathrm{C} / \mathrm{cm}^{2} \ldots \ldots \ldots \ldots$

4.7 SEM results for exposure of $80 \mu \mathrm{C} / \mathrm{cm}^{2}$ with sputtered $\mathrm{Au} \ldots$. . . . . . . . 50

$4.850 \mu$ m square with no lines $\ldots \ldots \ldots \ldots$

$4.90 .5 \mu \mathrm{m}$ lines at exposure of $70 \mu \mathrm{C} / \mathrm{cm}^{2}$ as seen under the optical microscope . . . 61

4.10 SEM images of $0.5 \mu \mathrm{m}$ lines in resist bilayer written at an exposure of $70 \mu \mathrm{C} / \mathrm{cm}^{2} \quad 62$

$4.110 .4 \mu \mathrm{m}$ lines at exposure of $60 \mu \mathrm{C} / \mathrm{cm}^{2} \ldots \ldots \ldots \ldots$

4.12 Some features at $60 \mu \mathrm{C} / \mathrm{cm}^{2} \ldots \ldots \ldots \ldots \ldots \ldots$

4.13 Process development for RIE samples . . . . . . . . . . . . . . . 66

4.14 Photoresist fabrication of gratings . . . . . . . . . . . . . . 67

4.15 SEM results with $C F_{4} \ldots \ldots \ldots \ldots \ldots \ldots$

4.16 SEM results of $S F_{6}$ etch with photoresist as mask $\ldots \ldots \ldots$

4.17 SEM results of $S F_{6}$ etch (2 mins.) of $10 \mu \mathrm{m}$ lines $\ldots \ldots \ldots$

4.18 SEM results of $S F_{6}$ etch(4 mins.) of $10 \mu \mathrm{m}$ lines $\ldots \ldots \ldots \ldots \ldots$

4.19 Etch depth plot for $10 \mu$ m lines with varying period $\ldots \ldots \ldots$. . . . . . 71

4.20 SEM results of low bias power(140W) $S F_{6}$ etch with photoresist as mask . . . 72

4.21 Plot of etch depth as pressure is varied $\ldots \ldots \ldots \ldots$

4.22 SEM results of high bias power(200W) $S F_{6}$ etch with photoresist as mask . . . 73 
4.23 Pattern in evaporated chrome after lift-off . . . . . . . . . . . . 75

4.24 Etch with sputtered chrome as mask . . . . . . . . . . . . 76

4.25 Etch with evaporated chrome as mask . . . . . . . . . . . . . . 76

4.26 Etch at $\mathrm{RF} 250 \mathrm{~W} \ldots \ldots \ldots \ldots \ldots \ldots$

4.27 Etch at ICP $100 \mathrm{~W} \ldots \ldots \ldots \ldots \ldots \ldots$

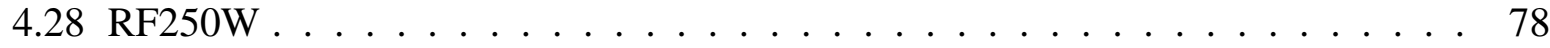

4.29 Etch at RF200W ICP 50W . . . . . . . . . . . . . . . . . 79

4.30 Plot of etch rate as RF power varies . . . . . . . . . . . . . . 79

4.31 Plot of etch rate as ICP power varies . . . . . . . . . . . . . . 80

4.32 Post-RIE ionic clean and HF dip . . . . . . . . . . . . . . . . 81

4.33 SF6 etched, acid cleaned and CF4 etched $\ldots \ldots \ldots \ldots$. . . . . . . . . 81

4.34 Etched silica with smooth surface $\ldots \ldots \ldots \ldots$. . . . . . . . 82

4.35 EDAX of etched silica and borofloat . . . . . . . . . . . . . . 82

4.36 Etched alumina . . . . . . . . . . . . . . . . . 83

$4.37 \mathrm{Si}$ etched with $\mathrm{Pt}$ as mask . . . . . . . . . . . . . . . . 84

4.38 Ablated depth as focus lens position is varied $\ldots \ldots \ldots$. . . . . . . . . 85

4.39 Ablated depth with increase in stage speed $\ldots \ldots \ldots$. . . . . . . . . . 86

4.40 Ablated depth for different no. of passes . . . . . . . . . . . . . 86

4.41 Measured vs. desired line spacing . . . . . . . . . . . . . . . . 87

5.1 Gratings diffracting white light . . . . . . . . . . . . 88

5.2 Optical set-up to obtain inteference fringes . . . . . . . . . . . . . . 89

5.3 Intensity comparison of Interference fringes of optically flat and etched Borofloat 90

5.4 HeNe light transmitted through etched Borofloat . . . . . . . . . . . . . 90

5.5 Intensity comparison of Interference fringes when HeNe light transmitted through air and etched Borofloat . . . . . . . . . . . . . . . . . . . 91

5.6 Optical set-up to vary wavelength $\ldots \ldots \ldots$. . . . . . . . . . 92

5.7 Intensity with varying wavelength $\ldots \ldots \ldots$. . . . . . . . . . 93

5.8 Optical set-up varying angle of diffraction $\ldots \ldots \ldots$. . . . . . . . . . . . 93 
5.9 Diffraction angle measurements for three angles of incidence . . . . . . . . . . 94

5.10 Diffraction of HeNe spot . . . . . . . . . . . . . . . . 94

5.11 Reducing HeNe spot size . . . . . . . . . . . . . . . . 95

6.1 Research Process Development . . . . . . . . . . . . . . . . 97

6.2 Gratings coupling light into waveguide . . . . . . . . . . . . . . 97

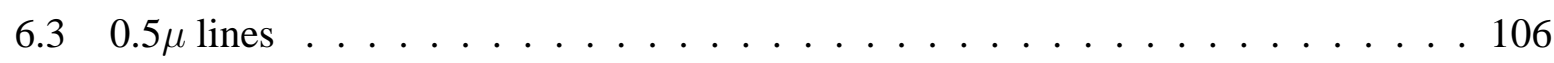

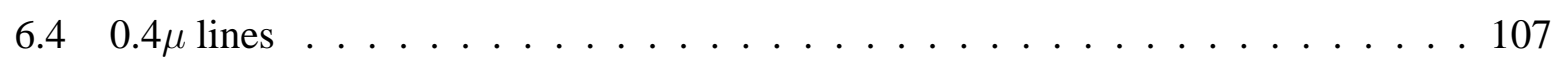

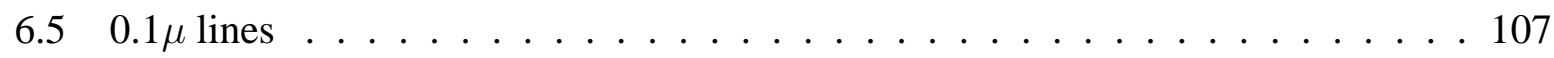

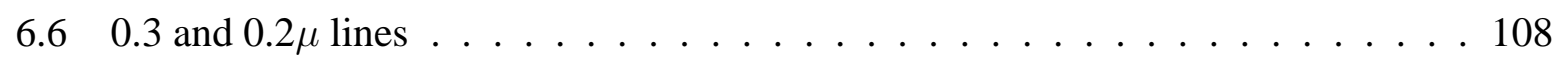

$6.70 .1-0.5 \mu$ lines in PMMA at different exposures $\ldots \ldots$. . . . . . . . . 109

$6.80 .4 \mu$ lines at $80 \mu \mathrm{C} / \mathrm{cm}^{2}$ in PMMA/copolymer . . . . . . . . . . . . 110

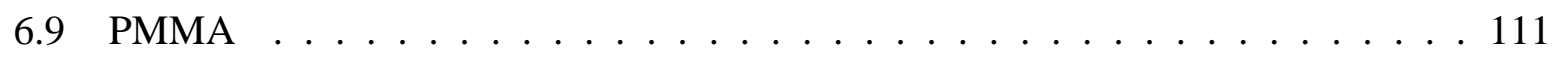

6.10 Single pass writes on PMMA/copolymer sample $\ldots \ldots \ldots \ldots$. . . . . 111 


\section{List of Tables}

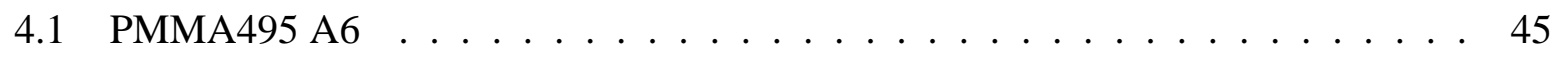

4.2 PMMA495 A6 Line $1 \mu \mathrm{m}$ Spacing $0.5 \mu \mathrm{m} \ldots \ldots \ldots \ldots$

4.3 PMMA495 A6 Line $0.5 \mu \mathrm{m}$ Spacing $1 \mu \mathrm{m} \ldots \ldots \ldots \ldots$

4.4 Pattern layout M1: $0.1 \mu \mathrm{m}$ - M5: $0.5 \mu \mathrm{m}$ Dose in parentheses $\ldots \ldots \ldots$

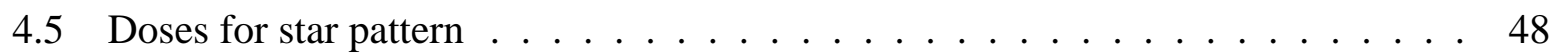

4.6 Lines with $50 \%$ duty in PMMA495 A6 . . . . . . . . . . . . . . . . 49

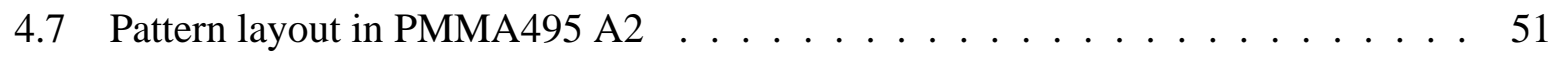

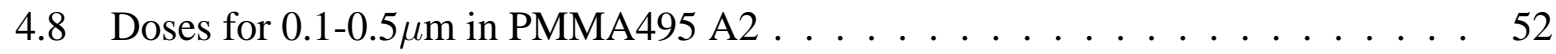

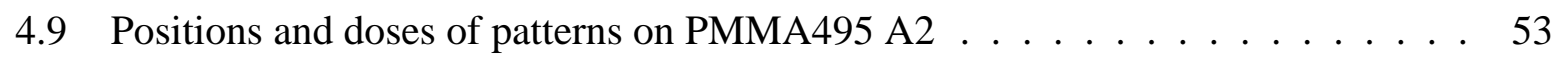

4.10 Exposures for different line sizes in PMMA495 A2 . . . . . . . . . . . 53

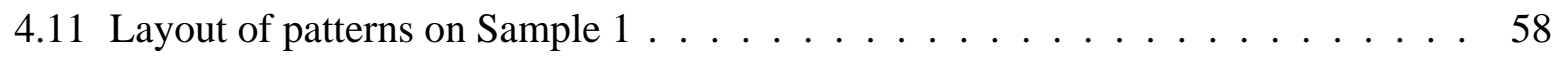

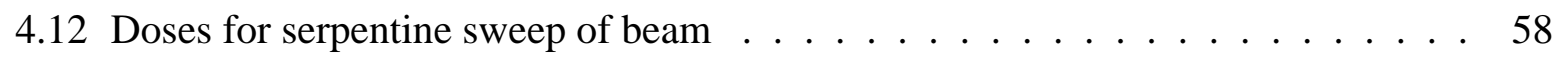

4.13 Doses for single pass of beam . . . . . . . . . . . . . . . . . 59

4.14 Modified doses for serpentine sweep of beam on PMMA A2 . . . . . . . . . 60

4.15 Modified doses for single sweep of beam on PMMA A2 . . . . . . . . . . 60

4.16 Doses for serpentine sweep of beam on bilayer $\ldots \ldots \ldots$. . . . . . 61

4.17 Doses for single sweep of beam on bilayer . . . . . . . . . . . . 61

4.18 Doses for 0.4 and $0.3 \mu \mathrm{m}$ lines using serpentine sweep of beam . . . . . . . . 63

4.19 Doses for $0.2-0.4 \mu \mathrm{m}$ lines using single pass of beam $\ldots \ldots \ldots$. . . . . . 63

4.20 Modified doses for 0.3-0.5 $\mu \mathrm{m}$ lines using serpentine sweep of beam . . . . . . 64

4.21 Modified doses for $0.2-0.5 \mu$ m lines using single sweep of beam $\ldots \ldots$. . . . 65 


\section{Chapter 1}

\section{Introduction}

\subsection{Background and Applications}

Gratings are used to couple light, for their focusing abilities, as sensors and various other optical elements. Diffraction gratings are used in monochromators, spectrographs and other optical instruments. They are also used with planar optical waveguides as input-output couplers, beam splitters, modulators, wavelength demultiplexers etc. Waveguide gratings are used in semiconductor lasers as distributed bragg reflectors. In optical fibers gratings are usually written inside the core of the fiber. Lamellar gratings, with the zero order of the wavelength eliminated, are used in compact disk heads where the zero order is used for data reading and first orders are used to maintain tracking and focus. Integrated grating structures are used for optical monitoring in MEMS devices [2]. There has been an increasing need to create gratings that have nanometer dimensions for applications that involve integrating them as light couplers in various micro devices. The prism coupler used in [3] and [4] can be replaced by a grating etched into one of the layers of the biosensor device as shown in Fig.1.1. The grating can also be used along with other diffractive optical elements as integrated monitoring systems for microfluidic channels where the gratings are created on the bottom surface of the substrate. 


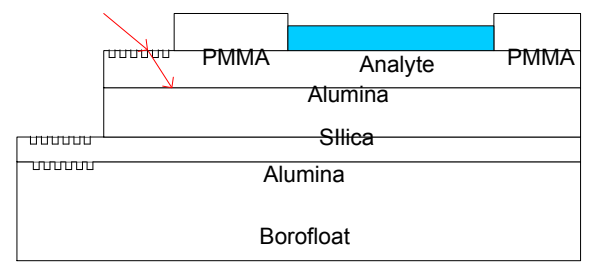

Figure 1.1: Possible locations of gratings to couple light into the Alumina waveguide

\subsection{Review}

\subsubsection{Methods of Manufacture}

Though it was David Rittenhouse who originally discovered the diffraction grating, it was Joseph Fraunhofer who announced his discovery when he created gratings using fine wire wound on a rectangular frame. His first ruled grating was in gold foil. Gratings were then created by ruling in glass with a diamond point. The improved ruling machines of today we owe to Henry Rowland. Gratings of greater size and precision could be ruled. However, ruling is a slow process and the errors incurred can be eliminated by other higher precision methods.

Ruling gratings of very small sizes (few hundreds of nanometers) is not a viable method. There are many alternate methods to manufacture grating structures. One is by the interference of two monochromatic collimated beams - holographic recording. Another is photolithography which can be used to transfer the grating pattern from a mask on to a photosensitive layer followed by etching the underlying material to create different kinds of gratings; reflective - metal or transmissive - glass. Direct methods of creating gratings include the use of either a laser or a high energy beam like an electron-beam (e-beam) system. The direct method using a laser has applications in creating gratings in fibers with monitoring capabilities. The e-beam method is important when the feature sizes are beyond the limits of photolithography. The only limitation is the rate of writing. Gratings can also be created by use of a phase-mask. The type of grating created with a phase mask would depend on the substrate material used. 


\subsubsection{Grating Types}

Gratings can be classified based on several criteria like geometry, material, method of manufacture, usage etc. Amplitude and phase gratings change only the amplitude and phase respectively of the incident light in different groove regions. Another classification is phase and relief gratings where phase gratings consist of refractive index variations and in relief gratings there is a surface relief structure that separates two media of different optical properties. A more important distinction type is reflection or transmission gratings. Reflection gratings are usually relief gratings covered with a highly reflecting material whereas in transmission gratings the back face needs an anti-reflection coating to prevent losses due to reflection and to prevent scattering in the substrate. In a symmetrical grating the normally incident light is divided with equal efficiencies into the symmetrical orders while in blazed gratings (triangular grooves) when light is incident normal to one of the facets, almost all of it is diffracted in the backward direction. Gratings can be divided depending on groove form into lamellar (rectangular), sinusoidal, triangular and trapezoidal gratings. Echelettes are blazed gratings with low working orders while echelles can have working orders up to several hundreds. From the method of manufacture is the obvious classification of ruled, lithographic and holographic gratings. Commonly used gratings are plane gratings where there are straight and equidistant grooves on a plane substrate. However, some times the grating period may be intentionally varied (chirped) to introduce some focusing properties. Curved and chirped grooves can be created on a concave substrate combining the dispersion properties of plane gratings with the focusing properties of concave mirrors. The classification or division of gratings into different types can also be based on their application.

\subsection{Research}

In this thesis fabrication methods for sub-micron gratings (200-500nm) are explored. As such small features are hard to obtain by photolithography with the available 320nm Mask Aligner, alternate methods of e-beam writing and writing with a 255nm Copper Vapor Laser (CVL) have been used. 
The grating lines are to be written on an e-beam resist spun on borofloat. After developing the resist a metal is deposited. The resist is then lifted-off to leave a grating pattern in the metal. The patterned metal is then used as a mask for etching the glass.

A bilayer resist process was used in e-beam lithography. The undercut in such a layer assists in lift-off. Processes for reactive ion etch of the materials of interest, borofloat, silica and alumina, are developed.

The laser is used to demonstrate its ability to ablate glass and create grating structures. It can also be used as a UV exposure tool to transmit UV light through a phase mask to create phase and relief gratings in glass and resist respectively. The phase mask will cause a periodic refractive index variation in the photosensitive glass. Relief gratings in photoresist will be obtained on development of the resist after exposure through the phase mask.

The paths followed during the research is shown in Fig.6.1.

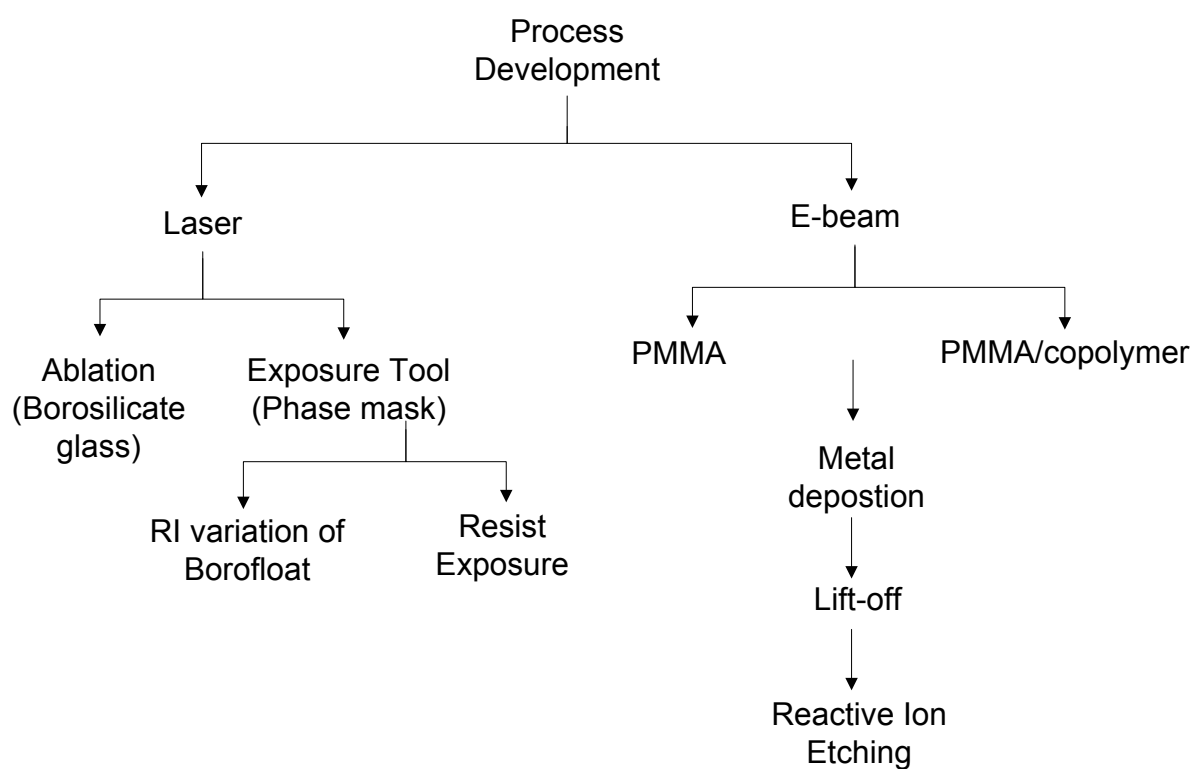

Figure 1.2: Research Process Development 


\subsection{Organization of Thesis}

This chapter gives an overview of the principle of diffraction, the types of gratings and their methods of manufacture and a few grating applications. The research carried out has been briefly outlined. The subsequent chapters will delve into the details. Chapter 2 explains the basic principles/theory of a diffraction grating along with details of some of the methods of manufacture. Chapter 3 discusses the methods of fabrication used in this research. The process steps, experiments and research carried out are included. Chapter 4 contains SEM images and explains the results obtained during each fabrication process step. Chapter 5 gives the results obtained on optical testing and shows the methods of characterization of the fabricated gratings using the HeNe laser. In Chapter 6 the research work is summarized and suggestions and recommendations are made for further work in improving the processes developed for fabrication of grating structures. 


\section{Chapter 2}

\section{Diffraction and Fabrication Methods}

\subsection{Principle of Diffraction}

Light bends when it is obstructed. This may be due to an edge or when it has to pass through a slit. This bending of light is called diffraction. Diffraction can occur with any type of wave including sound waves and water waves.

Thomas Young's double-slit diffraction experiment easily explains the concept of diffraction. Light is made to pass through two slits of narrow widths compared to the wavelength of incident light wave. Visualizing the light wave as a water wave simplifies the understanding. Two overlapping semicircles of waves are created on passing through the two slits. Constructive interference occurs where a crest overlaps with a crest and a trough overlaps with a trough to double the height of the crest and the trough respectively. Destructive interference occurs when a crest and trough overlap as they cancel out.

The pattern of light and dark fringes is seen in Fig.2.1(a). The intensity pattern is a multiplication of the interference pattern multiplied by the single slit diffraction envelope.

In Fraunhofer diffraction the incident light is assumed to be parallel and the imaging plane is at a large distance from the diffracting object. Fresnel diffraction refers to the general case making it more complex mathematically.

A diffraction grating is an example of multiple-slit Fraunhofer diffraction.

\footnotetext{
${ }^{1}$ (a) Available at http://en.wikipedia.org/wiki/Diffraction and (b) Available at http://hyperphysics.phyastr.gsu.edu/hbase/phyopt/mulslid.html/c2
} 


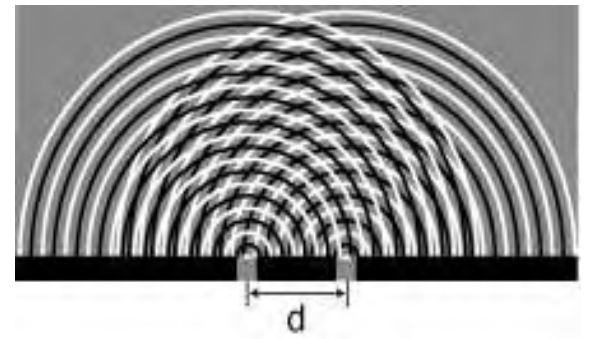

(a) Double slit diffraction

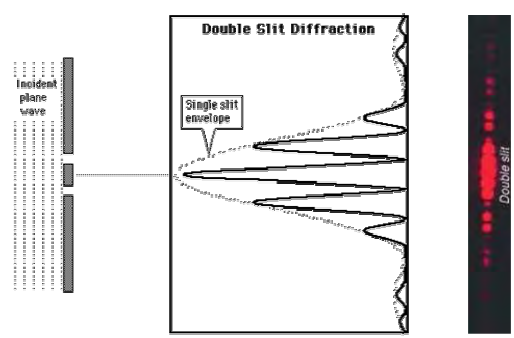

(b) Double slit intensity

Figure 2.1: Double-slit Diffraction ${ }^{1}$

\subsection{Grating equation}

A plane wave incident at an angle $\Theta_{i}$ with respect to the grating normal is diffracted from the grooves as shown in Fig.2.2.

The geometrical path difference between the light diffracted by successive grooves is

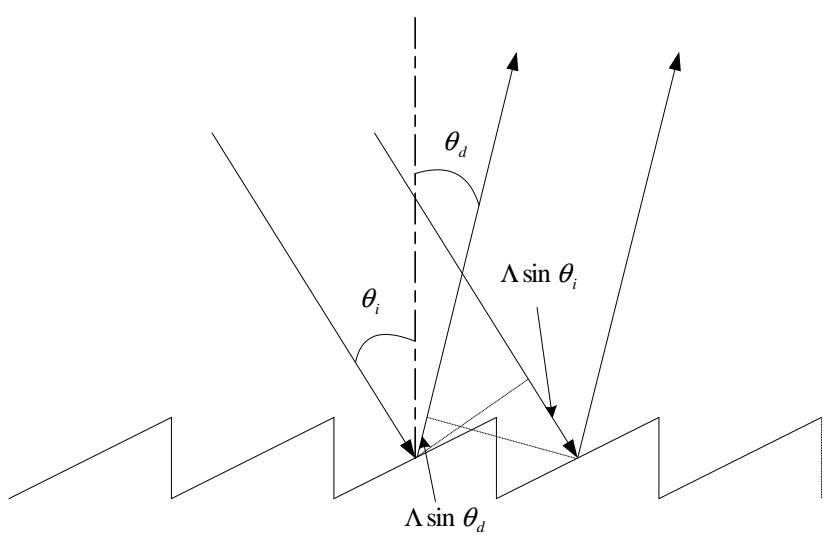

Figure 2.2: Phase Angle Relation in a Grating ${ }^{2}$

$\Lambda\left(\sin \Theta_{i}-\sin \Theta_{d}\right)$ where $\Lambda$ is the groove spacing. The difference in phase between two neighboring rays is then $2 \Pi \Lambda\left(\sin \Theta_{i}-\sin \Theta_{d}\right)$.

Thus, the light in the two diffracted rays will be in phase only when the path difference is an integral multiple of the wavelength of light. At all other wavelengths there will be destructive

\footnotetext{
${ }^{2}$ Diffraction Gratings and Applications, E.G. Lowen and E.Popov
} 
interference.

$$
\Lambda\left(\sin \Theta_{i}-\sin \Theta_{d}\right)=m \lambda
$$

where $m=0, \pm 1, \pm 2, \pm 3, \ldots$ This is the grating equation.

The angles of incidence and diffraction are often treated with opposite signs when they lie on either side of the grating normal. There are various sign conventions and since $m$ takes both positive and negative values, there are a number of ways the grating equation is written.

Both the incident and diffracted beam lie on the same side of the normal to the transmission

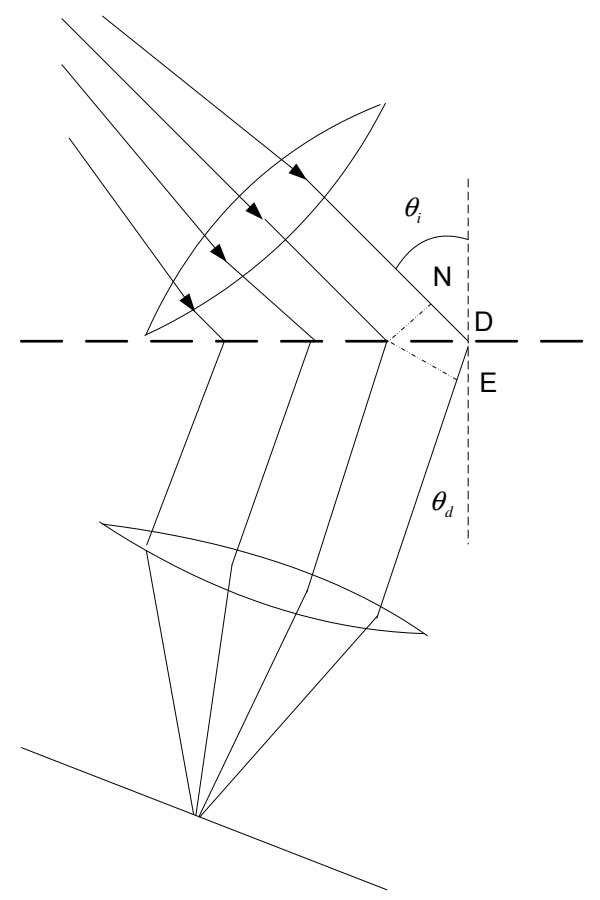

Figure 2.3: Phase Angle Relation in a Transmission Grating ${ }^{3}$

grating as shown in Fig.2.3. The path difference of corresponding points of adjacent lines is ND+DE. The principal maximum of the $m$ th order of the wavelength $\lambda$ is formed when $\mathrm{ND}+\mathrm{DE}=\mathrm{m} \lambda . \mathrm{As} \mathrm{ND}=\Lambda \sin \Theta_{i}$ and $\mathrm{DE}=\Lambda \sin \Theta_{d}$, the grating equation takes the form

$$
\Lambda\left(\sin \Theta_{i}+\sin \Theta_{d}\right)=m \lambda
$$

\footnotetext{
${ }^{3}$ The Diffraction of Light, X-Rays, and Material Particles, Charles F. Meyer
} 
When both the angles approach $90^{\circ}$, the path difference approaches twice the grating space. The wavelength $\lambda=2 \Lambda$ is the greatest wavelength a grating can diffract.

For a particular wavelength, all values of $\mathrm{m}$, for which $|m \lambda / \Lambda|<2$, correspond to physically realizable diffraction orders. Thus, the light is prevented from being diffracted in more than a finite number of orders. Specular reflection $(\mathrm{m}=0)$ is always possible, i.e. zero order exists. Light of wavelength $\lambda$ and diffracted in $m=1$ order will coincide with $\lambda / 2$ diffracted in $m=2$ order. Hence, filtering (order sorting) is required as the detector can't distinguish between the different wavelengths incident on it.

Blazed and lamellar gratings:

The property of gratings to concentrate the diffracted light into a specific order is called blazing. It is perfect when no light goes elsewhere. In this grating the grooves have a sawtooth profile. The phase across each individual groove is varied by introducing an amount of optically dense material such that each contribution is in phase. By controlling the shape of the grooves a grating can be blazed for any order.

A lamellar (laminar) grating consists of grooves with rectangular cross-section (Fig. 2.4).

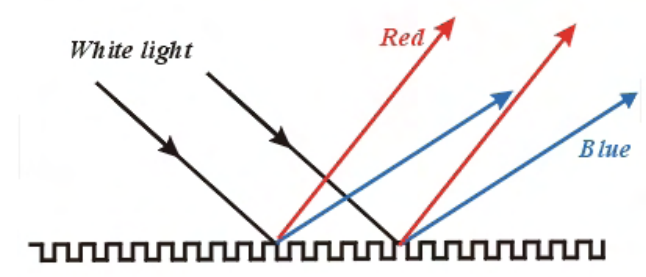

Figure 2.4: Diffraction of white light by a Lamellar Grating

When the height of the grooves is such that the optical path difference between the rays reflected (or transmitted) at the top and on the bottom is half the wavelength of the light, the zero order of this wavelength is eliminated, while equally strong first orders appear at either side. Such a grating is useful as a beam splitter and is often used as a phase mask. In a reflection laminar 
grating the groove height is one quarter of the wavelength for zero order suppression.

Phase mask:

A phase mask is a surface relief grating etched in fused silica (Fig.2.5(a)). In most cases,

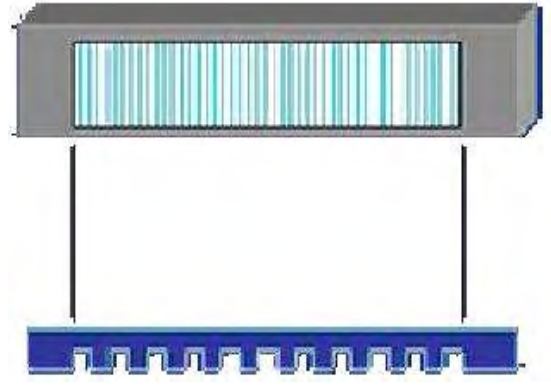

(a) Phase mask $^{4}$

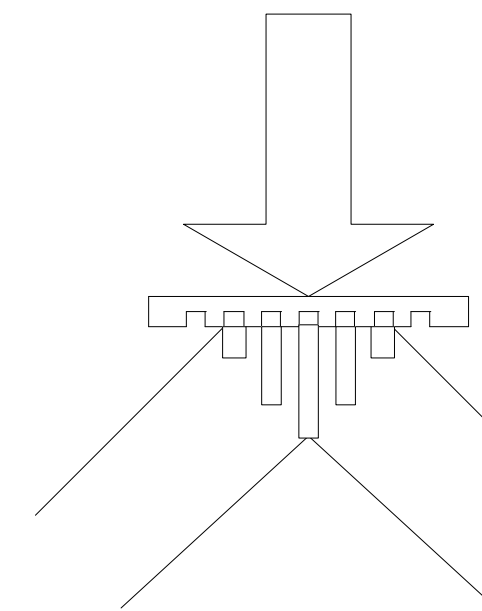

(b) Interference pattern

Figure 2.5: Phase mask to generate interference pattern

the phase mask is a diffractive optical element used to split the incoming monochromatic beam (usually UV) into two outgoing beams. By generating two outgoing beams, a phase mask creates an interference pattern located in the space where the two outgoing beams overlap (Fig.2.5(b)).

Most phase masks are fabricated in UV-transparent fused silica of high purity. The period or pitch can range from a few hundred nanometers to a few microns. The phase mask is usually $1 / 4 "-1 / 8 "$ thick. The grating profile is usually rectangular.

Phase masks are used in various applications but are most frequently used to record other gratings. They are used to create planar waveguide gratings, in integrated optics devices and widely in fiber Bragg gratings (FBG) where the grating is created in the core of the optical fiber. Only the resonant light frequency is reflected back while the others will pass through (Fig.2.6).

\footnotetext{
${ }^{4}$ Available at http://www.stockeryale.com
} 
FBGs are integral to fiber sensor devices and are commonly used in telecom components.

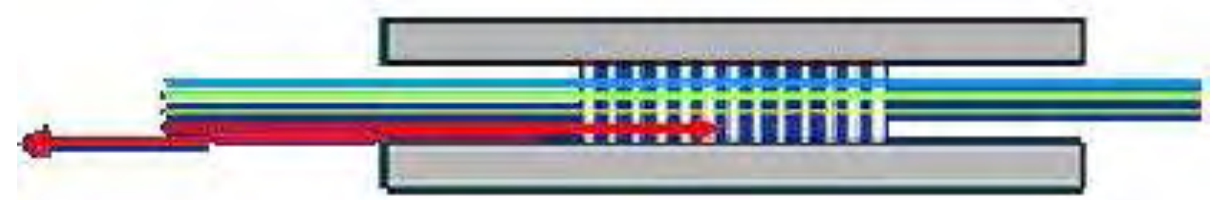

Figure 2.6: Fiber Bragg Grating ${ }^{5}$

\subsection{Fabrication Methods}

Gratings can be fabricated in many ways. The direct method of making gratings is by mechanical ruling. Holographic (interferometric, phase mask) method uses the interference of beams to create the grating structures. Photolithography can be used to transfer these structures into a variety of materials using a number of process steps. Direct methods of writing use a high energy electron beam or laser beam to expose/ablate the material.

\subsubsection{Mechanical ruling}

The traditional method of generating master gratings is by burnishing one groove at a time with a diamond tool. The smallest groove that can be written depends on the precision of the mechanical ruling engine. This method is still used but is limited by the size of the diamond tip and cannot be used for very small line sizes.

\subsubsection{Photolithography}

Photolithography can be used to transfer the required pattern onto a mask (photoresist itself or with further process steps to a metal), which can then be used to etch the grating pattern on to a substrate(Fig.2.7). Grating structures can be fabricated in different kinds of material with this process.

\footnotetext{
${ }^{5}$ Available at http://www.stockeryale.com
} 
In optical lithography a photomask contains the image of one of the layers in the process of

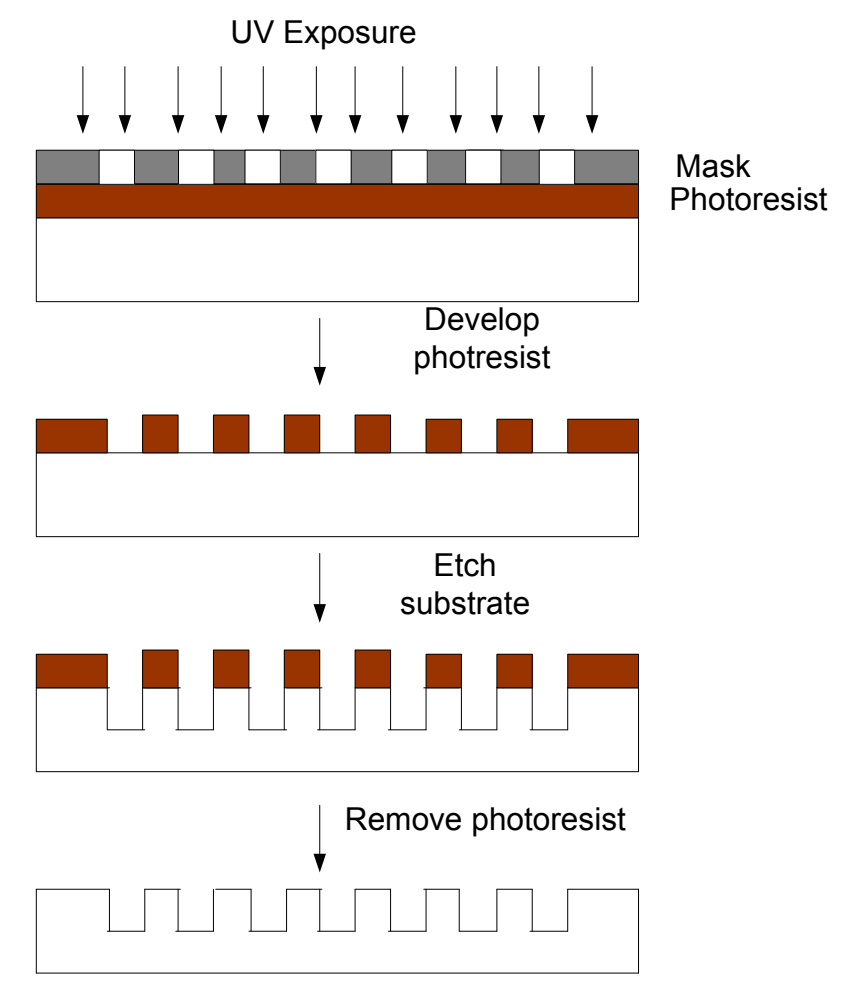

Figure 2.7: Photolithographic processing and etching

fabrication. An optical source is used to shine light through the mask. The image is projected onto the substrate, which is coated with a photosensitive material known as photoresist[5]. The photoresist has three components: a resin or base material, a photoactive compound (PAC), and a solvent that controls the mechanical properties of the photoresist such as the viscosity of the base, keeping it in a liquid state [5]. In positive resists, the PAC acts as an inhibitor until it is exposed to light, when it becomes a sensitizer and dissolves the exposed resist in a developer solution. Negative photoresists respond in the opposite way. The unexposed regions will dissolve in the developer, while the exposed regions remain. Image reversal resists (like AZ5214) are positive resists from which the negative can be obtained. However, positive resists tend to have the best resolution and are therefore more popular in fabrication.

Polymethyl methacrylate (PMMA) is a positive deep UV resist. It is used in applications where resolution is important. The carbon-carbon double bond in methyl methacrylate 
$\mathrm{CH} 2=\mathrm{C}(\mathrm{CH} 3) \mathrm{COOCH} 3$, naturally polymerizes to form PMMA, a long chain polymer consisting of alternating segments of $-\mathrm{CH}_{2}-$ and $-\mathrm{C}\left(\mathrm{CH}_{3}\right) \mathrm{COOCH}_{3}-$ [5](Fig.2.8). Under deep UV exposure the long chains can be broken and the resulting short molecules are more easily developed in a developing solution. PMMA has two primary drawbacks: it has very low etch tolerance and has a low sensitivity. It cannot be used as a mask in etching and optical exposures with very high doses of the order of a few hundred $\mathrm{mJ} / \mathrm{cm}^{2}$ are needed. PMMA is most commonly used as an e-beam resist.

Optical lithography cannot be extended for small features of the order of few tenths of a micron.

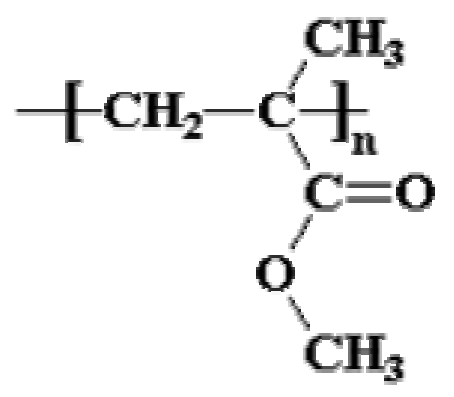

Figure 2.8: PMMA monomer

To achieve increased resolution, optical sources of shorter wavelengths(excimer lasers) and better resists (like PMMA and chemically amplified resists) are needed. Mask methods from use of phase shifting masks, binary (chrome) masks, attenuated phase masks to the presently being developed chromeless phase masks are trying to extend the optical lithography along with $248 \mathrm{~nm}$ and $193 \mathrm{~nm}$ sources to meet the $65 \mathrm{~nm}$ and $45 \mathrm{~nm}$ technologies' requirements.

Non-optical lithography techniques are used for feature sizes below $0.1 \mu \mathrm{m}$. Very shortwavelength energy sources are used: x-ray and electron beam. Resolution is good as diffraction no longer defines the resolution at these small wavelengths.

\subsubsection{Electron beam lithography (EBL)}

In electron beam lithography, the electrons interact with the atoms in the solid surface. The incident electrons may pass through undeflected, scatter elastically or inelastically. The scattering 
interaction can be treated classically as Rutherford scattering. A Monte Carlo simulation can be done to find the path of the inelastically scattered electrons in the substrate. The projected range of electrons can be calculated. Depending on the electron beam energy, the depth of penetration will vary. A high concentration of electrons with a wide range of energies is produced on exposure to the electron beam. The electrons penetrating the resist may cause unintentional damage to the substrate while intentionally causing chemical reactions in the resist Fig.2.9.

Photoresists used in e-beam lithography are long chain carbon polymers. One of two

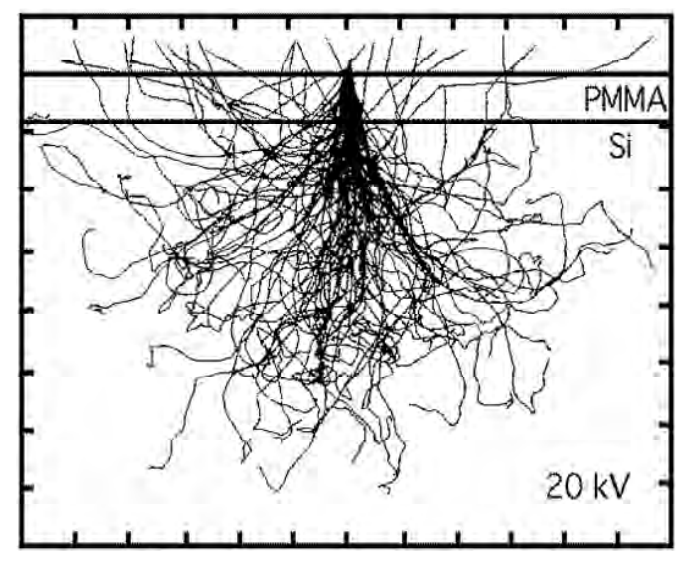

Figure 2.9: Monte-Carlo simulation for 20KV [1]

chemical changes may occur, cross linking and scission. In negative resists, atoms on adjacent chains are displaced and the carbon atoms bond directly i.e. cross link. The cross linked molecules dissolve slower in the developer solution. In positive resists, the e-beam radiation breaks the polymer chains (scission) such that they are more soluble in the developer. PMMA is a positive e-beam resist, so the exposed areas will be removed on development. This has been demonstrated by Stauffer et al.[6]. They have shown the effect of EHT voltage for direct write of PMMA 950K. Gratings with a period of 900nm were also created. Direct writing EBL in positive resist (SAL) was used to create both proximity-compensated gratings and uncompensated gratings in [7].

A disadvantage of the use of an energetic beam like the e-beam is that the beam is not confined to the resist layer. The beam or the secondary electrons may cause undesirable chemical changes 
in the underlying layer. However, in this research, the underlying layer is silicon/borofloat that is subsequently etched to transfer the grating structure into the substrate. Hence the high energy beam effects are not critical.

Electron beam lithography systems can be used to create masks or for directly writing the pattern on the substrate. An e-beam system needs an electron source with a high intensity, high uniformity, small spot size, good stability and a long life [5]. The cathode of the gun is the source of electrons and they can be obtained by heating it (thermionic), applying an electric field (field emission), a combination of the two (thermionic field emission), or with light (photo emission). The most commonly used source is thermionic due to its high intensity. Most thermionic sources use either tungsten or lanthanum hexaboride. The filament material must be chosen so as to minimize evaporation and have the longest life. An important figure of merit for the gun is emitted electron current density given by $J_{c}=A T^{2} e^{-\left(E_{w} / k T\right)}$ where A is the Richardson's constant $\left(10-100 \mathrm{~A} / \mathrm{cm}^{2}-K^{2}\right)$ for the material and $E_{w}$ is the effective metal work function [5]. The emitted electrons are accelerated and a fraction of them are collected. Increasing the emitted current density generally increases the brightness $\beta$. However if an increase in $\beta$ decreases the collection efficiency then the increase in brightness is not as large as the increase in current density.

Once an electron beam is generated, it must be focused into a narrow beam. This is done using electrostatic lenses and apertures. Most systems use Gaussian beams. The e-beam system we used is the Leica Cambridge SEM Stereoscan S360 that uses a thermionic cathode with a tungsten filament to create a Gaussian beam. To improve the throughput (primary disadvantage of e-beam lithography), special purpose systems have shaped beams generated by a combination of direct write and projection lithography [8]. However, in research applications and in manufacture of photomasks, where throughput is not a critical parameter, a simple e-beam system more than serves the purpose. Furthermore, an EBL system can be made at relatively low cost by employing a scanning electron microscope in write mode with appropriate software control. A Leica Cambridge SEM S360, enabled for e-beam writing using NPGS (Nanometer Patter Generator

\footnotetext{
${ }^{6}$ http://www.jcnabity.com
} 
Software $)^{6}$, was used in this research.

Proximity effects:

This is a major area of concern for electron beam lithography as it leads to pattern distortion. It is a name commonly used to describe effects caused by non-uniform electron exposure received in the resist due to a wide backscattered electron distribution initiated by incident electrons [9].The scattered electrons may expose nearby areas that are not meant to be exposed. It may cause unintentional exposure if two features are very close to each other. The proximity effect can be understood by considering a Monte Carlo simulation of electron trajectories of a typical e-beam exposure Fig.2.9.

Forward scattering occurs over a small range of angles and leads to only a slight broadening of image. Back scattering contributes a large area of exposure. To minimize the effects of this broadening, e-beam lithography is done in a thin resist layer and requires optimization of the incident beam for a particular resist layer. The back scattering range can be quite large. Depending on the resist, this energy dispersal may be sufficient to reduce the exposure dose without any changes to the properties over the area. What one has to be careful with is the overlap of trajectories when the patterns are very close to each other, which may lead to unwanted exposure. In the case of the gratings in this research, which are essentially line and space alternately patterned, this is a big hurdle, especially for a feature size of $0.1 \mu \mathrm{m}$.

A number of algorithms have been developed that can be used to modify exposure time to compensate for the proximity effects. In [7] proximity compensated gratings were created and compared to uncompensated ones. The compensation was made by an iterative inverse-filter processing of the electron-beam point spread function which resulted in proximity compensated dose data [7]. Mihir Parikh developed a computer program, SPECTRE, a self-consistent proximity effect correction technique for resist exposure [9]. The number of electrons (density) that should be incident in a region for identical average resultant exposure is calculated. The proximity function and pattern are inputs and the corrected pattern data (dwell time) is the output of SPECTRE. Further developments and details can be found in [10] and [11]. A pixel 
dose correction technique using the simplex method, a mathematical optimization technique, has been demonstrated [12]. It shows better contrast than GHOST, a non-mathematical technique. Another (more physical) solution is to use a bi-layer process: the lower layer is a copolymer (PMMA/MAA) and the upper layer is the resist (PMMA). PMMA/MAA is the more electronsensitive layer. The increased sensitivity results in an undercut which is necessary for a liftoff process. More importantly, the lower layer of copolymer separates the PMMA from the substrate and thus reduces the degradation of the resolution of the pattern in the PMMA due to backscattering electrons [13].

In this thesis a copolymer-PMMA bilayer is used to create under-cut patterns which would subsequently facilitate lift-off.

\subsubsection{Laser Ablation}

Another direct-write method of creating gratings is by using a laser to ablate lines in a material. Instead of using it as a source of light as in the holographic method, its intensity can be used to ablate the substrate (especially glass surfaces). In this method, each groove is created one at a time as in mechanical ruling.

Ablation is the process of using a high energy beam to melt the surface and remove it by vaporization. The substrate should absorb at the wavelength of the laser. At 250nm the absorption coefficient of borofloat glass is very high. The absorbed energy heats the material. If the absorbed energy is high enough it melts the material in the interaction zone. If the energy is higher, the material vaporizes and a high pressure is created in the interaction zone causing explosive ejection of the vaporized material. The molten material is ejected in the form of micro-droplets. Heat is deposited in the material and the interaction zone gets hot. Energy density of the beam determines the nature of the machining process. There are broadly three regimes:

1. Low energy density: At low energy, the material melts, but as the energy is low for expulsion, very little is vaporized and produces a shallow feature as it re-solidifies. This material may be loosely attached and may crack on cooling. 
2. Medium energy density: At the ideal energy density, the pressure wave produced removes the material and a reasonable depth is obtained. The feature is clean and there is little or no recast material and little or no damage to the surrounding material.

3. High energy density: The feature may be only slightly deeper than a medium energy density as the absorption depth of light determines the depth in the melt zone. However, micro-cracking may be caused due to energetic micro-explosions. Also it is difficult to control the lateral feature as melting and ejection take place outside the main interaction zone. The extra heat deposited around the feature causes very rapid heating of the part and may cause cracking due to thermal shock. A poorly defined feature with damage around it results.

The time required for this process is determined by the repetition rate of the laser pulses and the scribe speed. The scribe speed also determines how much material is removed i.e. the depth of the channel/groove produced. The depth of the line/groove produced is a function of the number of pulses overlapping at any one point. A deep groove can be produced by scribing slowly and a shallow one by scribing fast or alternatively, a deep groove can be obtained by repeated fast scribes over the same path. However, high repetition rates can cause melting of surrounding and, in some cases, underlying material. Hence, to get good lines, the speed should be reasonably high while avoiding melting. Multiple passes can be used to create deep grooves. The scribe speed should be adjusted such that the temperature rise around the feature is sufficient to soften but not melt the glass with the effect of annealing the stressed glass around the feature. The scribe speed is a function of material properties, mainly, thermal conductivity and melting point.

The resolution of the gratings created by this method is limited by the smallest beam spot that can ablate at reasonable scribe speeds. The spot size depends on the aperture used and the focusing lens position.

\subsubsection{Interferometric Fabrication}

In the holographic interferometer two beams overlap each other and form an interference pattern on the substrate. The substrate in most cases is photoresist or photosensitive silica as 
in the case of fiber Bragg gratings. The grating period, which is identical to the period of the interference fringe pattern, depends on the writing wavelength, and the half angle between the intersecting UV laser beams. The advantage of this approach is that the gratings are free of the periodic and random groove placement errors of mechanically ruled gratings. It is also faster than ruling as the grooves are formed simultaneously. This approach is also used to create fiber Bragg gratings with various lengths and various periods.

The disadvantages of this method are:

1. It is very sensitive to environmental conditions such as vibrations, alignments and setup configurations.

2. It is very difficult to control the resonant wavelength of the Bragg gratings.

3. It requires good coherence of UV laser and good stability of UV laser power.

4. The optical recording and the following development of the photoresist do not give precise control of the groove form.

When the lack of precision is not too important, the photoresist (and FBG) gratings win over the ruled ones.

The interferometer can be created in many ways; the two coherent beams can be obtained by a beam splitter, mirrors, a prism, a phase mask or any combination of these optical components.

In this thesis, the use of a $255 \mathrm{~nm}$ copper vapor laser and a phase mask is explored as in [14][15]. The first advantage of the phase mask is that it is not sensitive to environmental conditions, such as vibrations. The second advantage is that it still requires the coherence of the UV laser source. The third advantage is that the outgoing beam results are very consistent and do not depend on the setup configurations. The sole disadvantage is that different phase masks are required for gratings of different periods.

There are two typical modes in which phase masks are used; $+1 /-1$ and $0 /-1$. In the $+1 /-1$ mode, the UV is directed normal to the phase mask and the outgoing beam is separated into two diffraction orders, +1 and -1 , with an equal power level. The period of the interference pattern is 
one half the period of the mask. In the 0/-1 mode, the UV is directed towards the phase mask at an angle of incidence such that the beam is diffracted into $0 /-1$ orders and the period is the same as that of the mask.

Phase Mask in $+1 /-1$ Mode:

Normal incidence of UV radiation generates a pattern of fringes i.e. stationary alternating

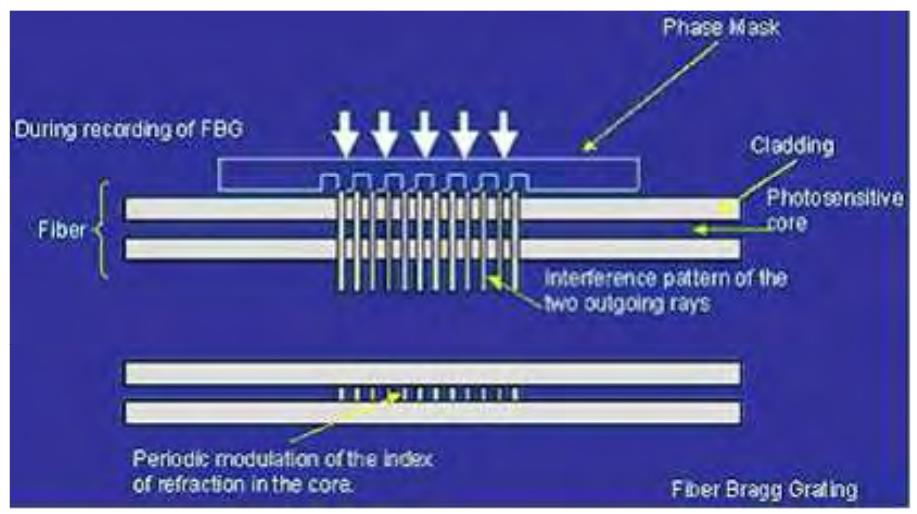

Figure 2.10: Recording FBG in $+1 /-1$ mode $^{7}$

zones of high and low intensity (represented by vertical lines), by the interference of the two outgoing beams. When a piece of optical fiber is placed in the interference pattern, the core of the fiber which is made of photosensitive material (usually doped with oxides of boron, phosphorus, germanium etc.) shows a periodic refractive index modulation caused by the exposure to UV light. This is how a FBG is created as shown in Fig 2.10. Surface relief gratings are obtained when resist is used as the exposed areas are removed on development.

The angles of diffraction $\Theta_{0}, \Theta_{-1}, \Theta_{+1}, \Theta_{-2}, \Theta_{+2}$ etc. are given in terms of the UV wavelength $\lambda_{u v}$ and the phase mask period $\Lambda_{P M}$, by the formula

$$
\sin \Theta_{m}=m \lambda_{u v} / \Lambda_{P M}
$$

The period of the fringe pattern is one half that of the phase mask and is independent of the source wavelength. $\Lambda_{\text {grating }}=1 / 2 \lambda_{P M}$

\footnotetext{
${ }^{7}$ Available at http://www.stockeryale.com
} 


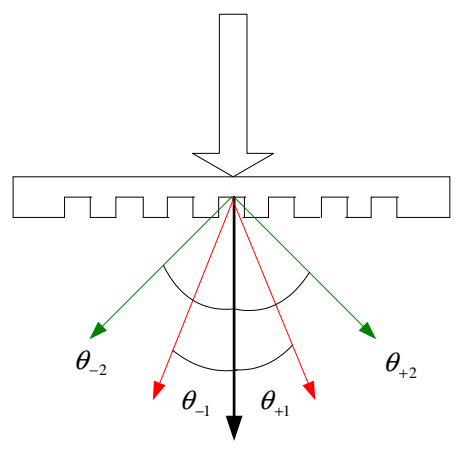

Figure 2.11: Phase mask in $+1 /-1$ mode

Phase masks can be optimized so that the intensity in the $+1 /-1$ mode is maximized while that in the zero mode is minimized. This can be achieved when the smallest etch depth in the phase mask is

$$
d\left(n_{P M}-1\right)=\lambda_{u v} / 2
$$

Also the intensity of the higher orders, if present, is minimized.

Phase mask in 0/-1 Mode:

To ensure that the interference fringes are perpendicular to the phase mask surface, $\left|\Theta_{0}\right|=$

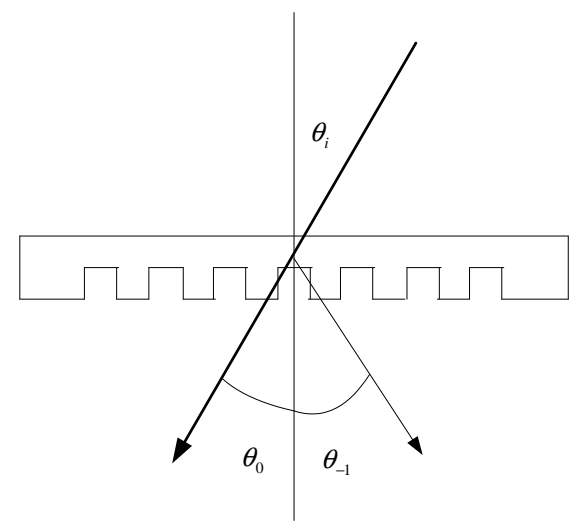

Figure 2.12: Phase mask in 0/-1 mode

$\left|\Theta_{-1}\right|$ must be satisfied. The required angle of incidence is $\left|\sin \Theta_{i}\right|=\left|\sin \Theta_{0}\right|=\lambda_{u v} /\left(2 \Lambda_{P M}\right)$ 
Moreover, if the condition $2 / 3 \Lambda_{P M}<\lambda_{u v}<2 \Lambda_{P M}$ is satisfied, then the incident wave is diffracted into only a single order (the -1 order) and no other orders (such as $+1,2,3$, etc.). In other words, there are two and only two outgoing beams: the transmitted 0 order and the diffracted -1 order.

The period of the fringe pattern created by the interference of the 0 order and -1 order beams is exactly equal to the period of the phase mask period $\Lambda_{\text {grating }}=\Lambda_{P M}$. This remains true regardless of the wavelength of the incident radiation, and regardless of whether the condition $\left|\sin \Theta_{i n}\right|=\lambda_{u v} / 2 \Lambda_{P M}$ is perfectly achieved.

In this thesis, the phase mask in the $+1 /-1$ mode is explored as it has a simple optical arrangement as shown above. The phase mask is placed on the photosensitive material and a 255nm Copper Vapor Laser (CVL) is used as the UV source. 


\section{Chapter 3}

\section{Research Fabrication Methods}

\subsection{Choice of Substrate}

Borofloat glass is the substrate used for both direct write lithography and the laser-phase mask methods. E-beam lithography with a bi-layer was developed on a silicon substrate and can be similarly done with borofloat substrate by changing the dose/fluence. The Schott Borofloat 3" wafers are used in the Bioagent Detection Biosensor project. By creating gratings in the same substrate, they could be integrated to couple light into a waveguide. In addition to its low price, the reasons for the choice of Borofloat for the Biosensor project can be found in [3]. Borofloat is a borosilicate glass made by the float process. It is a clear and transparent glass and is resistant to water, acids and alkalis as well as organic substances. Fig.3.1 shows the chemical composition of Borofloat. The presence of metal oxides poses a problem in reactive ion etching (RIE) as seen in subsequent chapters.

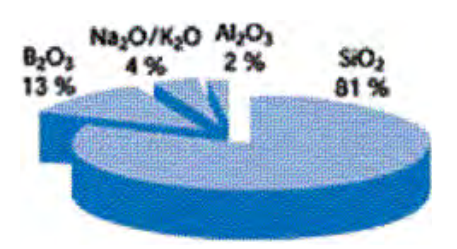

Figure 3.1: Chemical properties of borofloat ${ }^{1}$

\footnotetext{
${ }^{1}$ Available at http://www.us.schott.com/hometech/english/products/borofloat/attribute/chemical/index.html
} 


\subsection{Direct-write and RIE}

\subsubsection{Grating depth}

Grating structures with period of 500nm down to 200nm have been patterned in PMMA by e-beam lithography. To nullify the zero order, the etch depth for $632 \mathrm{~nm} \mathrm{HeNe}$ from equation 2.4 is $d=632 / 2((1.47-1))=672.3 \mathrm{~nm}$. This was the depth to which borofloat was etched by RIE.

\subsubsection{Creating gratings by direct write and reactive ion etching}

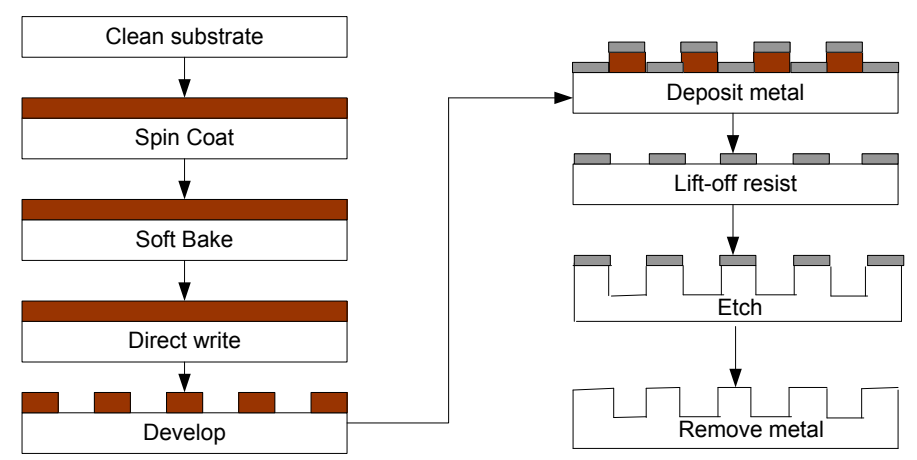

Figure 3.2: Process steps of fabrication of gratings by e-beam and RIE

Direct write in PMMA and lift-off processing of evaporated metal have been used to create metal gates [16]. EBL in PMMA and RIE with chrome as a mask to create pillars, ridges and trenches has been demonstrated [17]. In this research gratings have been created by writing the pattern in PMMA using e-beam, evaporating chrome, followed by lift-off and then plasma etching into the glass substrate.

The steps developed and followed to create grating structures are shown in Fig.3.2 and explained in the following sections. 


\subsubsection{Substrate clean}

The gratings are to be created in borofloat glass. However, for process development silicon wafers that were cleaved to size were used. The size was dependent on that of the SEM holder. 8 samples can be loaded in the $\mathrm{SEM}$, each of about $2 \mathrm{~cm}$ X $2 \mathrm{~cm}$. A diamond scribe was used to cleave the samples. An organic clean was done (acetone-5 mins. methanol-5 mins.) and the samples were baked for 30 mins. at $100^{\circ} \mathrm{C}$ to get rid of all the volatiles.

\subsubsection{Spin on resist}

PMMA is most commonly used as a high-resolution positive resist for direct write e-beam process $^{2}$. Positive resists have the best resolution and hence are more popular in IC fabrication. PMMA has a high resolution, can be spun to a thinner film and it has a high rate of scission. When used for direct writing, PMMA is typically used as an imaging resist for a liftoff process. Ridges $50 \mathrm{~nm}$ wide have been created in Si using a 70nm layer of PMMA for patterning by EBL [17]. Gratings with periods of $600 \mathrm{~nm}$ and different profile depths have been written in resist (PMMA) using e-beam [6]. The e-beam write process development in this research began with PMMA495 A6 as it was available. But the thinnest film that can be spun is a little more than $300 \mathrm{~nm}$ at a spin speed of 4000rpm as shown in the plot of Fig 3.3.

Early write attempts of lines went all the way down to the silicon. Exposures of $150 \mu \mathrm{C} / \mathrm{cm}^{2}$ and above seemed over-exposed. In the next attempt, with equal line and space, all the resist was removed and squares were cut out in the resist. Over-exposure may not be the only cause. The e-beam write software (NPGS) does not include automated proximity correction. For a certain resist thickness, when 500nm wide lines and spaces were written, there could be parallel walls of resist that are $500 \mathrm{~nm}$ wide at the top and narrower at the bottom (due to undercutting). If the undercutting was too much, the walls could have been distorted or even fallen over as in this case. A lower dose may have helped the undercutting. But after reducing the dose, when observed under SEM, the lines had resist and did not go down to the silicon (under-exposed). To avoid undercutting (due to proximity effects in a thick film) and under-exposure (lowering

\footnotetext{
${ }^{2}$ Appendix 2 Microchem PMMA datasheet
} 


\section{PMMA A Resists \\ Solids: $2 \%-6 \%$ in Anisole}

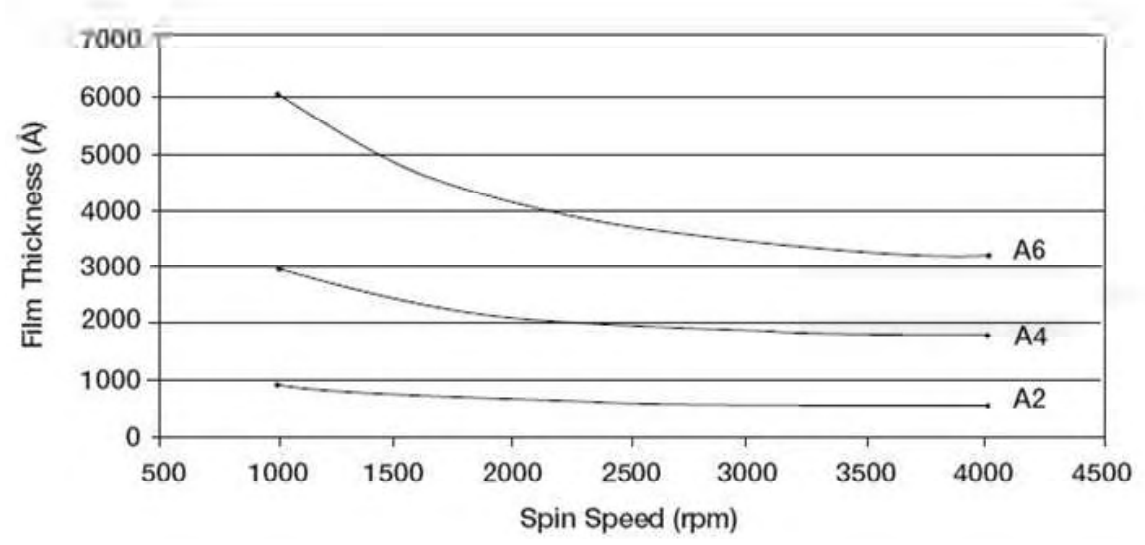

Figure 3.3: Film thickness of PMMA 495 A6 and A2 at different spin speeds ${ }^{2}$

exposure dose), a thinner resist film had to be used. Hence the need for PMMA495 A2 was felt. It can be spun on to have a film thickness of $90 \mathrm{~nm}$ down to about $50 \mathrm{~nm}$ as seen in Fig. 3.3.

The use of a copolymer (Copolymer MMA (8.5) MAA (methacrylic acid) EL6) with PMMA as a bilayer was explored. The copolymer is the more sensitive layer causing an under-cut profile in the bilayer. This assists the lift-off process as explained in 3.2.2.6. EL6 gives the thinnest copolymer film.

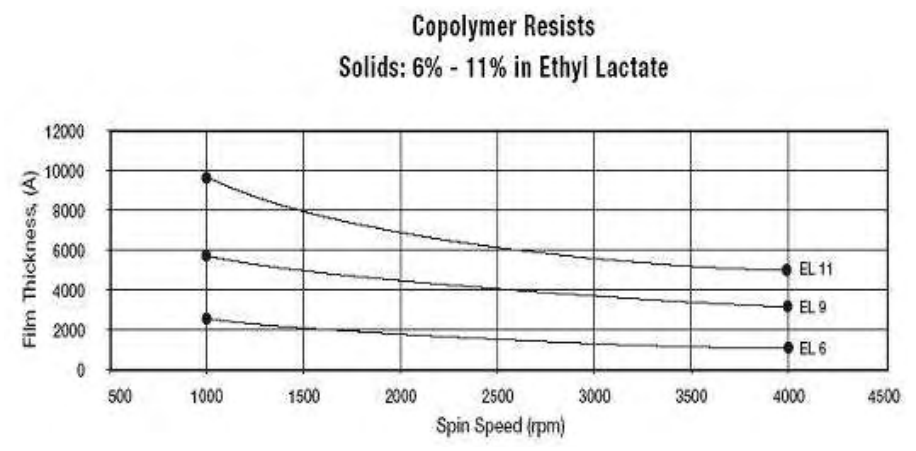

Figure 3.4: Copolymer resists at different spin speeds ${ }^{2}$

The lift-off process with and without copolymer is explained in detail in section 3.2.2.6. 
Additional metal layer to prevent charging: PMMA often exhibits a charging effect when placed under an SEM for viewing and it becomes necessary to coat it with a thin conductive layer. To prevent charging during the e-beam write, it is recommended to deposit a thin metal film [7],[18]. Charging of the substrate may cause pattern distortion. The metal layer is removed after the write and before developing the resist [7].

A 10nm Al layer was sputtered over the PMMA. After writing, the Al is to be etched using $\mathrm{NaOH}$ and the PMMA is developed ${ }^{3}$. But when this was attempted, PMMA was removed on development. This also happened with Al etchant Type A from Transene. Even diluted solutions of the etchants didn't work. As this was just a precautionary measure, it was decided to do without it at the present. This can be explored for improving the process in the future.

\subsubsection{E-beam exposure}

Though the direct e-beam method takes longer to write compared to photolithography, when very small features are needed, it becomes very difficult for photolithographic tools and processes to provide comparable results.

The e-beam write essentially requires an electron source and a pattern generator. This can be easily and cheaply achieved by interfacing software to an SEM. The pattern to be written is drawn in a CAD file. The NPGS software used has the capability of importing various file formats. The pattern can be created in any drawing software like DesignCAD and L-EDIT and files in .DWG or .CIF form can be created. Other file formats can also be used. However, one has to be careful when using a CAD tool besides the DesignCAD (file created with .dc extension) in the NPGS package, as some commands may not be converted appropriately in the run files (.rf6 extension). The NPGS software converts them to the ASCII .dc2 file format. In the DesignCAD, different patterns can be drawn with the various drawing tools. A single pass of the beam or a serpentine sweep (where the beam zig-zags to fill the feature drawn) can be selected. Before running the

\footnotetext{
${ }^{3}$ www.jcnabity.com
} 
file, which begins the write process, one must ensure that the SEM is set to the desired voltage and it is focused and its astigmatism has been minimized. The beam current should be set so that the beam spot is circular. The beam blanker voltage must be set such that it moves the beam off the sample and no emission is seen when it is turned on. In the Run File Editor, the appropriate .dc2 file (drawing file) is opened and either the required dose or exposure time is entered. The magnification and beam current have to be entered. The file is then saved as a run file (.rf6). The magnification on the SEM is adjusted and the SEM is then switched to write mode The pattern defined in the run file is then written by selecting the desired run file in the file list in the NPGS Menu and then the Process Run File option. The Leica Cambridge Stereoscan 360 SEM used is shown in Fig. 3.5.

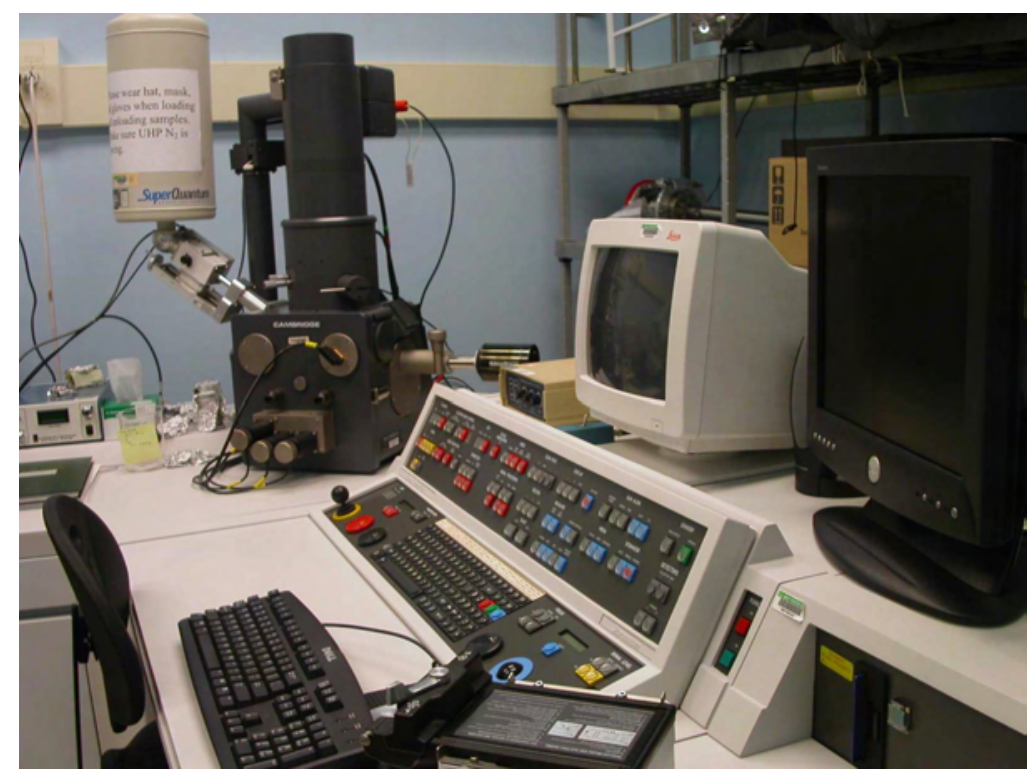

Figure 3.5: Leica E-beam system ${ }^{4}$

Specifications:

- Acceleration Voltage: 0.2-40KV

- Resolution: Tungsten (W) cathode-5nm

${ }^{4}$ Dept. of Physics, West Virginia University 
- Magnification: 5-300,000

- Detector: Secondary electron detector, backscattered electron detector

- Specimen chamber: 270x270x250mm

- Stage: Computer controlled motorized, movements $\mathrm{X} / \mathrm{Y}=100 \mathrm{~m}, \mathrm{Z}=70 \mathrm{~mm}$, tilt angle $=90^{\circ}$, rotation $=360^{\circ}$

- Accessories: High resolution display,optical and Winchester disk image storage systems, viewing window and Digital capture and image transfer software

\subsubsection{PMMA develop}

After writing the pattern, the exposed PMMA is to be removed. This is done by developing the resist in MIBK:IPA 1:1 for $30 \mathrm{sec}$ and then in 1:3 solution for $30 \mathrm{sec}$. Appendix 1. The 1:1 solution causes a faster development while the 1:3 solution slows it down. As explained in 2.3.3, the electron exposure reduces the average molecular weight and increases its solubility in the developer. So the exposed areas will dissolve and the line and space pattern will be created in PMMA.

So far, diffraction gratings in resist have been created and diffraction is seen when white light is incident on the gratings. Distinct colors are apparent at different viewing angles. The grating lines can also be seen under an optical microscope. PMMA and all other resists are temperature sensitive and have a short life. Hence, gratings in resist are not utilized. A more robust substrate is needed. The grating structures are transferred to the silicon substrate.

Sputtering: During the research, to ensure the e-beam write was optimized, the samples were viewed in the SEM. To prevent charging of the resist, which can cause pattern distortion, a metal layer was deposited by sputtering. This was done using the CVC 610 Sputter Deposition System. The steps to deposit platinum, about 30-35 nm thick (resist thickness of single PMMA layer is about $80 \mathrm{~nm}$ and a thicker metal may encapsulate all the features) are in Appendix 2.

A simple sputtering system is a parallel plate plasma reactor operated under vacuum. A high 
density of ions is generated and they strike the target material. These ions physically remove atoms of the metal being deposited. The target is placed on the cathode and the substrate on the anode. The cathode and anode are placed often closer than $10 \mathrm{~cm}$ so that all the sputtered atoms are deposited on the substrate. For this physical process, an inert gas like Argon is used to create the ions at pressures less than 1 mtorr.

\subsubsection{E-beam evaporation}

A mask is needed for the RIE process that follows. The gratings are etched in borofloat using RIE. PMMA and other resists are attacked at a faster rate by the gases used for the etch process (resist selectivity is very low) and thin resist cannot be used as a mask. Hence a metal, which has a lower etch rate, is used as the mask. Since the process of e-beam evaporation was already developed for chrome ${ }^{5}$, it was used as the masking metal. Chrome is also the most widely used mask for etch in the semiconductor industry. Normally a film stack of the substrate material, chrome and then resist is used. After e-beam write and development of resist, the chrome is opened by plasma etch after which the substrate is etched (Fig3.6).

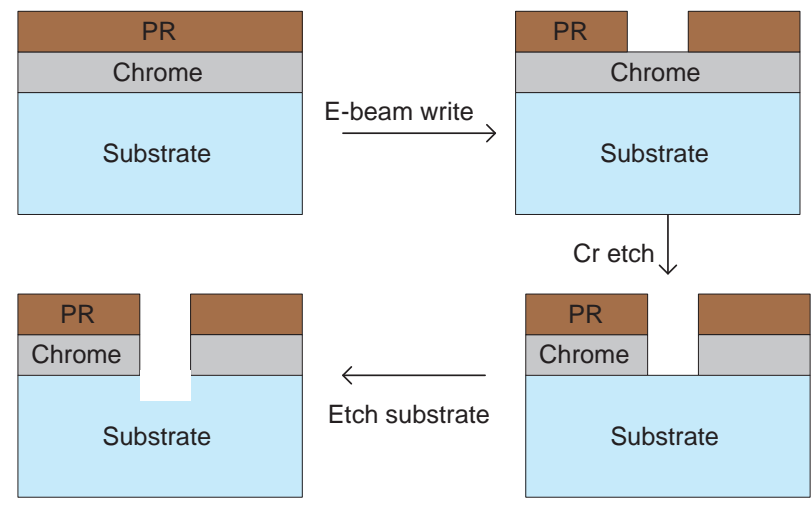

Figure 3.6: Chrome as mask for plasma etch

In this thesis, the focus has been on plasma etching borofloat. To dry etch chrome chlorine is required. The by-products of a chlorine etch are toxic. Due to the unavailability of suitable exhausting facilities, chlorine could not be used. Also wet-etching chrome would not be suitable

\footnotetext{
${ }^{5}$ Process developed by Timothy Cornell
} 
for the nanometer scale features. Hence developing the metal deposition and lift-off processes are necessary.

Evaporation gives poor step coverage. This is an advantage for the lift-off process. The sputtering process, with good step coverage, makes it tougher to remove chrome by lift-off. And hence the best way to deposit chrome is by evaporation. Contamination of the metal being deposited may be caused by resistive, inductive heating and thermionic guns. This is avoided by e-beam evaporation. In e-beam evaporation, the beam (generated by thermionic emission) is magnetically bent/ focused on to the top of the crucible containing the metal to be deposited. The metal evaporates and the atoms of the vapor travel in the chamber and accumulate as a film when they strike the surface. Rather than depositing and etching the metal layer (as stated above), the metal film is deposited on the patterned photoresist. The film naturally breaks at the edges of the resist. When the resist is removed, the metal film on top of the resist is also lifted off. Chrome 80 $\mathrm{nm}$ (has to be less than resist thickness for lift-off) thick was evaporated.

\subsubsection{Lift-off}

To remove chrome by lift-off, acetone was used to remove the PMMA and the chrome deposited on it leaving chrome in the exposed areas. Lift-off processing is commonly used for patterning evaporated films. When a single layer of PMMA is used for e-beam lithography, it is about 70-80nm thick. The metal to be used as mask for etching should be atleast 20-30nm thick to withstand the etch. The chances of the metal filling up the whole patterned feature is very high as shown in Fig.3.7. Lift-off would become difficult as the resist remover cannot attack the resist.

The ideal resist profile is shown in Fig. 3.8 [19]. When the metal film is deposited on such an undercut profile, it breaks at the edges and the resist remover can easily attack the resist. The resist is then removed along with the metal on top of it as shown in Fig.3.9. The undercut enables a clean separation at the edges. Though the use of PMMA as an electron-beam resist for lift-off processing has been explored by J.H Magerlein and D.J. Webb [19], it does not give an undercut edge profile. As mentioned earlier, this profile can be achieved by using a copolymer and resist 


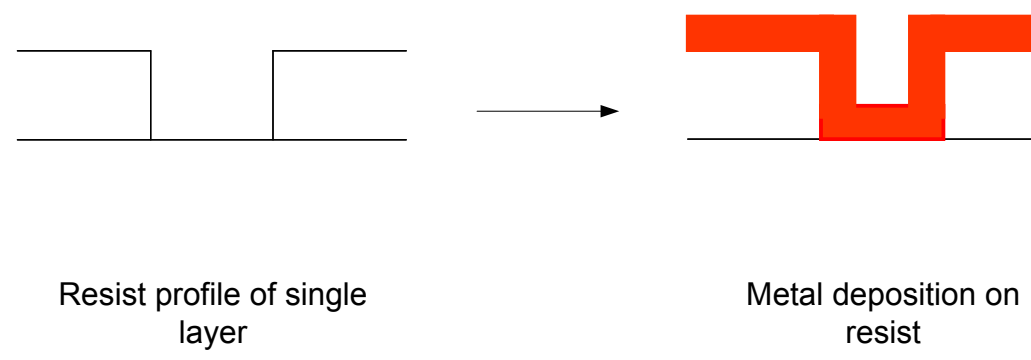

Figure 3.7: Metal deposition on single PMMA layer

bilayer process.

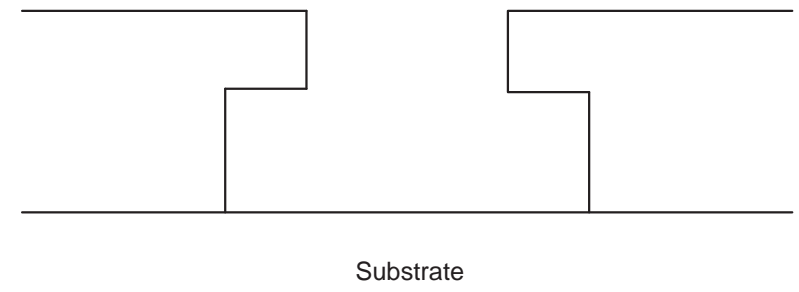

Figure 3.8: Idealized lift-off profile

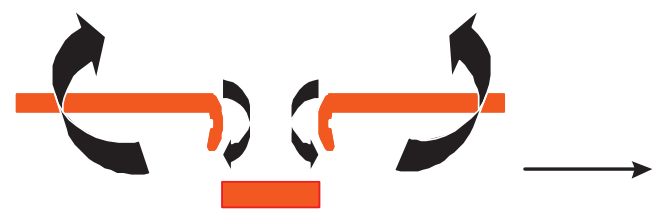

Figure 3.9: Metal deposition on bilayer

Copolymer resists can be created by copolymerizing the two monomers, methyl methacrylate (MMA) $\mathrm{CH} 2=\mathrm{C}(\mathrm{CH} 3) \mathrm{COOCH} 3$ and methacrylic acid $(\mathrm{MAA}) \mathrm{CH} 2=\mathrm{C}(\mathrm{CH} 3) \mathrm{COOH}$. The sensitivity, thermal stability and solubility in developers differ as the mol-\% of MAA in the copolymer is changed [20]. The copolymers have a lower molecular weight than PMMA resists and thus have higher dissolution rates in the developers. Copolymer MMA(8.5)MAA is commonly used

\footnotetext{
${ }^{6}$ Microchem PMMA datasheet
} 
with PMMA in bilayer lift-off processes and is also used in this research. Based on the process shown in the PMMA datasheet ${ }^{6}$ it was decided that the copolymer should be below the PMMA. 30nm features have been made with e-beam lithography using a two level resist system followed by reactive ion etching of silicon [21].

The upper layer is thin and is spaced above the substrate such that the effects of both forward scattering and back scattering are reduced (Fig.3.10). This separation and the undercut in the lower layer are ideal for lift-off processing. The use of a copolymer-resist bilayer to make the chrome lift-off easier is shown in Fig. 3.11.

The copolymer has been often used as a top layer, above the PMMA, as an imaging resist

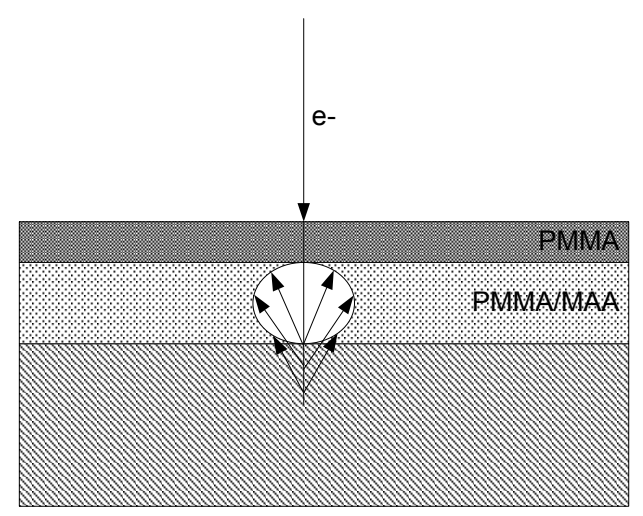

Figure 3.10: E-beam interaction in bilayer
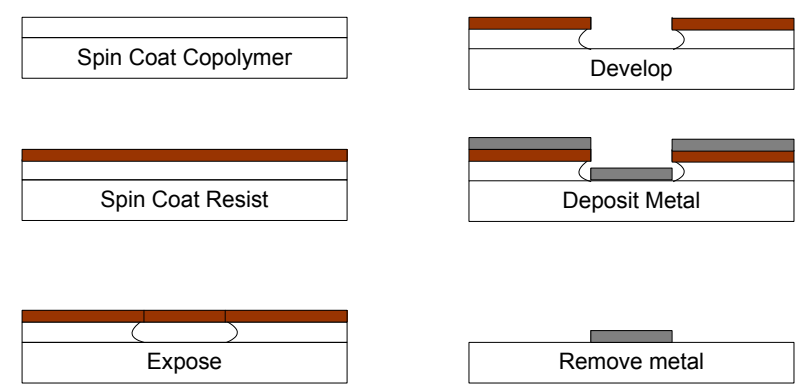

Figure 3.11: Copolymer-resist bilayer lift-off process

[19] and [22]. It is spun on thinner than the PMMA below. A shelf (undercut) or T-shaped structure can be created facilitating lift-off. Depending on the type of copolymer and resist and 
the different solutions used for development, the copolymer may etch faster or slower compared to the underlying PMMA layer. The use of a stack of different resists has been demonstrated [23]. In such cases, development of the different resists is in mutually exclusive solvents. An all-copolymer combination can also be used [20]. This is advantageous in high temperature applications like e-beam evaporation. The composite copolymer system can withstand higher temperatures than PMMA. As PMMA-MAA is the more electron sensitive layer, it has been used to obtain undercut in the composite resist profile [13]. In [13] 30nm thick gold is lifted-off and line widths of 40nm have been achieved.

The bilayer process increased the stack thickness and the e-beam write did not go all the way through in some instances. The hurdle was optimizing the dose. With the same doses as used before (on PMMA), the copolymer seemed to be overexposed. The grating lines were not created and there were empty squares. Either the copolymer was over-exposed and therefore lifted off the PMMA on development or due to the closely spaced lines the undercut of the copolymer from each side may have caused all the PMMA to lift-off. It was necessary to increase the thickness of the copolymer (almost four times that of PMMA).

Remover PG is recommended by Microchem ${ }^{7}$ to remove PMMA. But the resist strippers were to harsh and acetone had to be used.

Further investigation of development rates of resist and copolymer and their sensitivity is needed to explore the use PMMA-MAA (EL6) with PMMA (495 A2) as a bilayer stack to create sub-micron gratings. The solubility of the two layers needs to be studied. A diffusion barrier between the two films could be used. In such a case, a thin layer of evaporated metal is used. The optimal thickness of the copolymer as compared to the resist layer also needs to be ascertained. To successfully write lines 100-200nm wide the centers (of write process) may have to be increased i.e. the pitch (duty) of the grating should be reduced, so that there is room for undercut. For increased resolution, resists with higher molecular weight like PMMA950 may be used.

\footnotetext{
${ }^{7}$ www.microchem.com
} 


\subsubsection{Reactive Ion Etching (RIE)}

Etching is used to transfer a pattern onto the substrate using a mask. The mask may be photoresist or a hard mask (oxide or nitride) or metal (chrome). Etching is used to create contacts and vias in oxides, polysilicon gates, circuit lines in aluminum and numerous other applications.

Wet etching is usually isotropic where as plasma etching which uses fresh chemicals for each etch can be vertically anisotropic, allowing the patterning of narrow lines [24]. As the grating lines need to be $0.5-0.1 \mu \mathrm{m}$ apart, the etching has to be as anisotropic as possible. There is better profile control in plasma etching though selectivity is poor.

In plasma etching, a power source is applied in a chamber containing a gas to produce ions and reactive species (free radicals). Gases are used which, under plasma, will remove the film by forming volatile by-products. The volatile by-products are pumped away. The etch may involve generation of chemically reactive species (free radicals) from the feed gas molecules that react with the material to be removed or a physical etch where ions bombard the target and remove the film. Physical bombardment may be used to attain vertical profiles. Reactive Ion Etching is a combination of the chemical and physical etch. Ions and free electrons, created by ionization, provide the physical component of etch. The electrons are essential to sustain the plasma while the positive ions are important for etch. Free radicals provide the chemical etch component. A free radical is created when an electron collides with a molecule with sufficient energy to break it into a chemically reactive molecular fragment with an unpaired electron in its valence shell.

Plasma (glow discharge) is created by applying an electromagnetic energy to a gas. The characteristic glow is caused by the emission on photons. Electrons are excited to higher energy levels within the atom and emit photons when they return to their ground state. This light emission can be used to analyze the plasma and to determine etch endpoint. Initially, there are very few electrons in the neutral gas. A large voltage is applied causing the electrons to accelerate and collide with neutrals thus ionizing them. The newly produced electrons in turn accelerate and ionize more neutrals resulting in a cascading effect. With a sufficiently high voltage, the number of ions and electrons increase until an equi-potential cloud, plasma, is formed. Energy for the ionization is usually provided by RF electromagnetic fields. Strong electric fields and low 
pressures are required for ionization.

In capacitive coupling, the RF power accelerates the ions towards the cathode. The ionization process is inefficient and depends on secondary electrons. Anisotropy depends on ion bombardment. The higher the DC bias the higher the ion energy resulting in better directionality. Higher pressures result in more scattering and etch directionality decreases. Etch directionality becomes important as the features sizes get smaller. Thus, lower pressures are needed. However, if the pressure gets too low in the capacitively coupled chambers then the ion density may drop which may make sustaining the plasma more difficult. Magnetic field enhancement increases the ion density without increasing the ion energy. It confines the secondary electrons near the cathode and increases the probability of ionizing collisions. In inductive coupling, most of the RF energy is used in the ionization process. The RF power is transferred to the plasma via an RF magnetic field while the ionizing electric field is induced. It is more efficient in plasma production as no energy is spent in the acceleration of ions onto the surface. Combining capacitive and inductive coupling gives more independent control of ion density and ion energy than was possible with capacitive coupling even with magnetic enhancement. High density plasmas can be achieved at low pressures without high potentially damaging DC bias. The ICP power determines the ion density while the DC bias defines the ion energy.

The PlasmaLab 80+ RIE system used for etching can be used along with the ICP source. To transfer the pattern from the PMMA to the borofloat, chrome was used as the mask. Chrome is not etched (very slow etch rate) during RIE, whereas PMMA etches rapidly. The gases available were $\mathrm{CF}_{4}, \mathrm{O}_{2}, \mathrm{H}_{2}, \mathrm{CHF}_{3}$ and $\mathrm{SF}_{6}$. Fluorinated and chlorinated gases are commonly used as the etch by-products created are then volatile. The film to be etched determines the choice of gases. Selectivities to the underlying film and the mask will be another factor in choosing gases. If the reaction byproducts are non-volatile then the reaction is not very useful as the etchant won't get to the film as long as the by-products lie on the surface. On the other hand, film formers are required in processes for the purpose of profile or selectivity control. It is essential to balance the physical (ion bombardment) and chemical (etching by etchant species and deposition of film formers) aspects of the process. $\mathrm{CF}_{4}$ is an isotropic etchant by itself for $\mathrm{SiO}_{2} . \mathrm{O}_{2}$ will attack 
organics. It is used to strip resist and to clean the chamber walls after etching so as to remove any deposition.

Borofloat glass contains a small percentage of sodium, potassium and aluminum oxides. These form non-volatile products (fluorides). Various gas chemistries were tried. Quartz has been previously etched using $\mathrm{CHF}_{3}$ to create lenses [2]. Based on etch results on Pyrex using $\mathrm{CF}_{4}$ and $\mathrm{O}_{2}$ [25], and the similarity in chemical composition of Pyrex and Borofloat, the same recipe was attempted. However, micro-masking was observed (details in chapter 5). A low pressure recipe of $C F_{4}$ was then implemented. It still showed non-volatile compounds remaining on the substrate. To move closer to a physical etch, a low pressure and low flow plasma could be achieved with $S F_{6}$ [26]. The side-walls were vertical (anisotropic) and the physical etch removed most of the non-volatile by-products.

\subsection{Laser ablation and phase mask}

There are many techniques to fabricate gratings, such as photolithography, optical holography, electron beam lithography, laser writing, and mechanical ruling. In most of these techniques it is necessary to coat the substrate with either photoresist or an electron beam resist. It is then exposed and further processed for production of a grating profile. These are time-consuming and indirect methods for the fabrication of surface relief gratings. The mechanical ruling is a direct method but has a limitation on the feature size that can be ruled. The feature size or the line width of the grating could also be limited by the wavelength of the illuminating light as in the case of the lithographic techniques. In order to achieve smaller line widths and to do so in a short time, planar gratings have been fabricated by using Ti-sapphire laser to ablate the surface [27]. 10 micron lines in copper were created by using a galvano-scanner to control the number of pulses of the interference pattern (laser source) incident on the substrate.

The CVL can also be used to create gratings by interference lithography. It can be used to cause a refractive index variation in photosensitive material, or chain-scission in photoresist which on development can be used as a grating structure in itself, or as a mask for RIE.

The use of a laser to create gratings by ablation or by inducing a change in refractive index 
offers the potential for dry processing without the use of photoresist and etching.

The ideal laser should be able to manufacture gratings rapidly and reproducibly. It should have long coherence length, high beam quality, and sufficient power to enable writing in a reasonable time [28]. The excimer lasers, frequency-doubled argon ion lasers, frequencyquadrupled Nd:YAG or Nd:YLF lasers and frequency-doubled dye lasers, all fail to meet these requirements in one or more ways. For example, excimer lasers have limited coherence length, making them unsuitable for writing gratings using interferometric methods. Frequencydoubled argon lasers are limited in power. Frequency doubled, excimer-pumped dye lasers and frequency-quadrupled YAG or YLF lasers are similarly limited in average power, and their poor beam quality and high peak powers cause rapid degradation of mirrors, phase masks and other components. In contrast, frequency-doubled copper vapor lasers (CVLs) operating at $255 \mathrm{~nm}$ have a long coherence length $(40 \mathrm{~mm})$, high beam-pointing stability $(\sim 5 \mu \mathrm{rad})$, good spatial beam quality, and can produce high average powers (up to $1000 \mathrm{~mW}$ ) from a single oscillator [29]. The high repetition rate $(10 \mathrm{kHz})$ and relatively low peak power lead to long optical component lifetimes. Multiple fibres were exposed through a phase mask simultaneously to demonstrate that the UV-CVL is a suitable source for mass production of matched gratings [29] and [30]. In [30] the frequency-doubled laser CVL manufactured by Oxford Lasers was used to demonstrate that despite the $255 \mathrm{~nm}$ operation wavelength being off the UV absorption peak of the B/Ge co-doped fiber, single and multiple gratings can be fabricated.

CVL: In this research Oxfordlasers' MP100 255nm Copper Vapor Laser was used. Its specifications are as follows:

- Frequency Doubled Copper Vapor Laser- high power, high repetition rate $(6 \mathrm{KHz})$ with wavelength of $255 \mathrm{~nm}$ and power up to $1 \mathrm{~W}$.

- Telescope Optics

- PC controlled Processing Shutter 
- Safety shutter and Interlock circuits

- $\mathrm{PC}$ controlled $\mathrm{X}, \mathrm{Y}$ and $\mathrm{Z}$ axes

- $\mathrm{CNC}$ controllers, drivers

- Beam delivery system and mounts

- On-line camera system for alignment and monitoring of machining

- Data logging

Applications $^{8}$ : CVL is used to micro-machine silicon, nitrides, metals like stainless steel, alumina, diamond, ceramics and glass. Holes, lines, and other different shapes, arrays, high aspect ratio features, blind holes, can be created. It has been used to manufacture extrusion nozzles for fibers and textiles, fluid orifices in stainless steel for ink-jet printers, pinholes/slits in metals for applications like leak detection and gas/liquid filtering. It is used to scribe diamond, sapphire. It can be used to create microchannels and reservoirs in borosilicate glass.

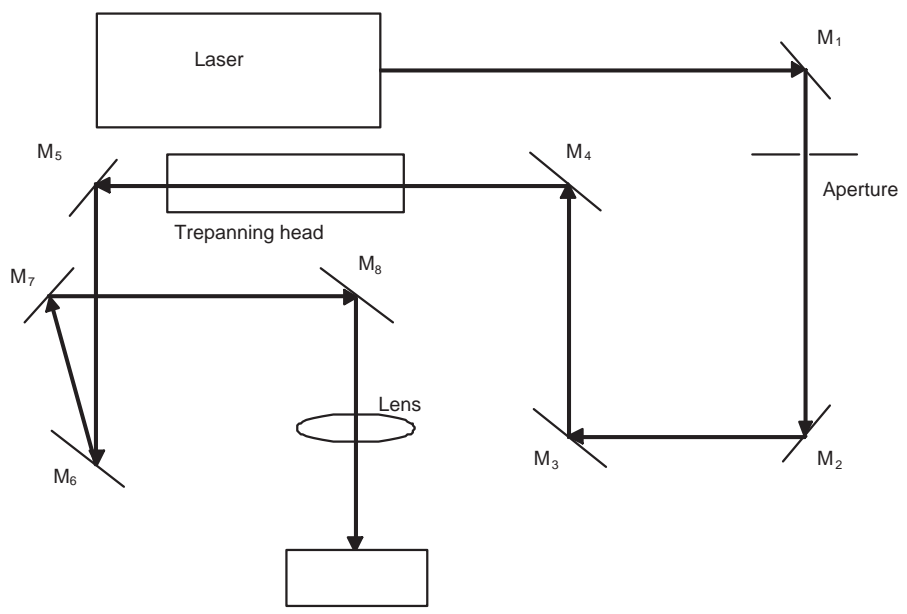

Figure 3.12: Schematic of laser system ${ }^{9}$

\footnotetext{
${ }^{8}$ www.oxfordlasers.com

${ }^{9}$ Timperman Group, CRL, Dept. of Chemistry, WVU

GaN Light Emitting Diodes and a study of etching techniques, Smiti Bhattacharya, Apr 2004
} 
Fig.3.12 shows the laser set-up in the MP100 CVL system. The spot size depends on the aperture used $(0.25-3 \mathrm{~mm})$ and the focusing lens position. The stage is moved relative to the beam. The speed of the stage can be controlled. With PC control the beam can be made to scan the same path more than once. The no. of passes and stage speed can be varied to control ablation depth.

\subsubsection{Grating period}

The grating period of ablated lines using the laser depends on the power, the aperture/mask size (beam size), the writing speed and the number of passes. When using the laser and the phase mask, the grating period is fixed and depends on the phase mask used. If the phase mask has a period of $1076.2 \mathrm{~nm}$, with normal incidence of light as explained in 2.3, the period of the grating will be half that of the phase mask, i.e. $538.1 \mathrm{~nm}$.

\subsubsection{Laser Process}

\subsubsection{Ablation}

The ability to create gratings by ablation using Oxfordlasers' 255nm Copper Vapor Laser $(\mathrm{CVL})$ was investigated. However, the smallest feature size was 8-10 microns wide with a $1 \mathrm{~mm}$ aperture (mask). By changing the aperture size down to $0.25 \mathrm{~mm}$ finer lines may be attainable. But with a smaller aperture the energy of the beam used in ablation reduces. The lines may not be ablated deep enough. Longer exposure, i.e. moving the stage slower or multiple passes, may be needed. In addition, the period of line and space may need to be increased to prevent over exposure of adjoining lines causing lines with reduced height as shown in Fig. 3.13.

Characterization of material is required before creating features. The absorption coefficient

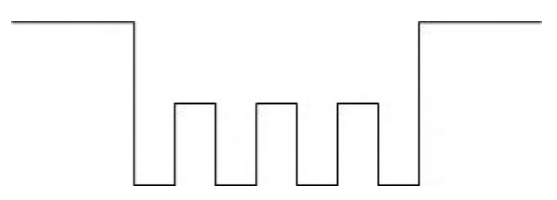

Figure 3.13: Reduced height of lines due to promixity 
for each material differs for a given wavelength $(255 \mathrm{~nm}$ in this case). The energy from the UV light breaks down the molecular bonds in the target material surface. Each material has its own photo-ablation threshold, below which photo-ablation does not occur. It is necessary to optimize focus, fluence, study re-deposition etc. First the right focus must be found. This can be done by ablating holes with the focussing lens at different positions. The lens position that gives the best(cleanest) hole is then used for subsequent micromachining. The hole reflects the beam size and its radius is then used to calculate the fluence.

$$
\text { Fluence }=\text { MeasuredPower }(W) / \text { BeamArea }\left(\mathrm{cm}^{2}\right)
$$

where Area $=\Pi r^{2}$.

To create a diffraction grating, a program was written to ablate lines. Parameters like speed of write, no. of passes of the beam were varied to obtain the best lines. The lines were viewed under a microscope for signs of micro-cracking of glass, deposition of ablated material, sidewall angle etc. To remove debris, ultrasound bath may be used or the sample can be polished (sandpapered) using lens paper. With higher fluence, the absorption depth increases resulting in a larger interaction volume which increases the heat counducted to the surrounding material. Thus a smaller interaction volume helps to control thermal damage.

\subsubsection{Phase mask method}

Lasers have also been used to create refractive index modulation in photosensitive substrates (glass). In [31], the interference of UV beams is used to expose the hydrogenated germaniadoped fiber. Gratings can also be created using chrome mask and exposing quartz to $248 \mathrm{~nm} \mathrm{KrF}$ laser causing similar refractive index variation as demonstrated for waveguide channels [31]. This method is advantageous as gratings can be written in any localized area of the waveguide. They are erasable by heating and can be rewritten. Grating of any period, size and shape can be written.

Similarly, the CVL can also be used as a source of UV light to create two coherent beams that interfere such that they create a fringe pattern. This pattern is translated onto the material as periodic line and space structures. The easiest method to create an interference pattern is to use 
a phase mask. The phase mask is placed directly above the substrate as close to it as possible. It can also be held at a distance and with the use of 2 mirrors the +1 and -1 modes can be made to interfere on the substrate/fiber. There are many ways of creating the interfering beams as in [27] where a glass plate and mirrors are used, whereas in [32] and [33] a prism is used. All these methods require a lot of optical components and must have a vibration-free set-up. The use of a phase mask is thus the simplest. Even with phase mask, the UV light can cause refractive index variation or cause ablation/scission depending on the type of substrate. The use of a phase mask to create gratings in photosensitive material by modifying the optical properties of the material has been demonstrated in [31] and [34]. It has also been used to ablate (copper) in [27]. In [35] and [36] gratings have been created in resist. The grating pattern in resist can be used as a resist mask in the fabrication of gratings in another substrate as stated in [36].

The only disadvantage of the phase mask is that it can be used only to create gratings of a fixed size and pitch.

With the available material and optical components, gratings in photoresist using the CVL and a phase mask were explored. A phase mask with period of 1.076 micron was used. With normal UV incidence, the grating created in resist should be about $533 \mathrm{~nm}$ (half the phase mask period as discussed in chapter 2). Various resists, AZ 4110, AZ5214E, PMMA 495A6 and PMMA 495A2 were tried. However the laser did not change the properties of the AZ resists as they are not deep-UV resists. Gratings can be created in PMMA by either varying the refractive index or causing chain-scission. The UV laser modification of the optical properties of PMMA has been demonstrated in [37]. Its application in plastic optical fibers is explored in [38]. Fiber gratings can be conveniently created. Creating waveguides and other devices and sensors in PMMA is possible.

Borofloat with $13 \% \mathrm{~B}_{2} \mathrm{O}_{3}$ and $81 \% \mathrm{SiO}_{2}$ is photosensitive to the $255 \mathrm{~nm}$ wavelength. A similar optical set-up as for resist would give grating (RI variation) of the same size $(533 \mathrm{~nm})$ in borofloat.

However due to time constraints and system failure, the phase mask method could not be demonstrated. 


\section{Chapter 4}

\section{Fabrication Process Development and Results}

\subsection{Direct write}

\subsubsection{PMMA495 A6}

\subsubsection{Varying line spacing}

PMMA 495 A6 was used initially. It was deposited on a square of silicon cleaved to size to fit in the SEM. The first pattern tried was the sample 'star' pattern in the NPGS software. The file contains a $3 \times 2$ array of the pattern shown in Fig.4.1. This was the recommended starting point to optimize dose. The file was run as is without altering exposure time or dosage.

The array was written with different dwell times 10-85 $\mu$ secs (doses from 53.5-455

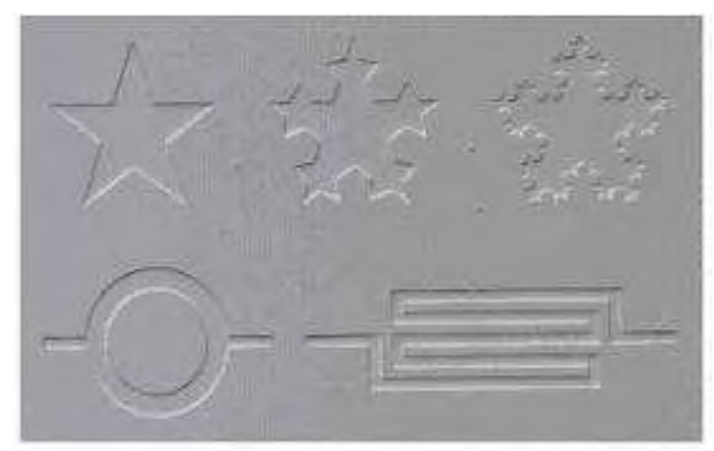

Figure 4.1: Star pattern

$\left.\mu C / \mathrm{cm}^{2}\right)$. 2 sets of lines were written with dwell times 12-52 $\mu$ secs $\left(\sim 60-260 \mu C / \mathrm{cm}^{2}\right)$. The 
dwell time is the exposure time per point. If the dwell is entered the corresponding exposure dose is calculated to match the time and vice-versa. Hence only one parameter needs to be varied. Dwell time was varied in the initial experiments. Later, dose was found to be a better metric over different pattern sizes. The dose can be entered as area dose, line dose or point dose ${ }^{1}$.

$$
\begin{aligned}
\text { Area Dose } & =\frac{(\text { Beam Current }) \times(\text { Exposure Time })}{(\text { Center }- \text { to }- \text { center }) \times(\text { Line Spacing })} \\
\text { Line Dose } & =\frac{(\text { Beam Current }) \times(\text { Exposure Time })}{(\text { Center }- \text { to }- \text { center })} \\
\text { Point Dose } & =(\text { Beam Current }) \times(\text { Exposure Time })
\end{aligned}
$$

Center-to-center (CC) : distance between adjacent exposure points ${ }^{1}$ Line spacing (LS) : distance between adjacent passes of the beam ${ }^{1}$

The patterns written were lines with space half the linewidth (line- $1 \mu \mathrm{m} / \mathrm{space}-0.5 \mu \mathrm{m}, 0.6 / 0.3$ and $0.2 / 0.1$ ) and lines with space double the linewidth (line- $0.5 \mu \mathrm{m} / \mathrm{space}-1 \mu \mathrm{m}, 0.3 / 0.6$ and 0.1/0.2). Lines were drawn in DesginCAD using polyfill/serpentine sweep.

8 samples can be placed in the SEM at a time. Each sample can be no wider than $2 \mathrm{cms}$ on each side. While using the SEM in write mode, one must know the position of the sample prior to writing. The sample cannot be looked at or focussed on as it will get exposed. To determine the sample positions in the SEM, they were noted when loading. Positions are determined by $\mathrm{X}, \mathrm{Y}$ and rotation. For example, two PMMA samples were placed in the SEM, Sample 1 was at rot $=5.39$ and sample 2 at $\operatorname{rot}=6.64$ for $\mathrm{X}=587.25, \mathrm{Y}=343$.

$\mathrm{EHT}=30 \mathrm{KV}$

Working distance $=26 \mathrm{~mm} \quad$ Filament current $\mathrm{I}_{f}=2.56 \mathrm{~A} \quad$ Probe $/$ beam current $\mathrm{I}_{p}=50.04 \mathrm{pA}$.

For the Star pattern:

Field $155 \mu \mathrm{m} \quad$ Magnification 647 CC-18.87nm $\quad$ LS-49.53nm

\footnotetext{
${ }^{1}$ NPGS Manual
} 
Table 4.1: PMMA495 A6

\begin{tabular}{|c|c|c|c|c|c|}
\hline \multicolumn{2}{|c|}{ Star 1 } & \multicolumn{2}{c|}{ Star 2 } & \multicolumn{2}{c|}{ Star 3 } \\
\hline $\begin{array}{c}\text { Dwell Time } \\
\mu \mathrm{s}\end{array}$ & $\begin{array}{c}\text { Dose } \\
\mu C / \mathrm{cm}^{2}\end{array}$ & $\begin{array}{c}\text { Dwell Time } \\
\mu \mathrm{s}\end{array}$ & $\begin{array}{c}\text { Dose } \\
\mu C / \mathrm{cm}^{2}\end{array}$ & $\begin{array}{c}\text { Dwell Time } \\
\mu \mathrm{s}\end{array}$ & $\begin{array}{c}\text { Dose } \\
\mu C / \mathrm{cm}^{2}\end{array}$ \\
\hline 35 & 187.28 & 60 & 321.06 & 85 & 454.83 \\
30 & 160.53 & 55 & 294.31 & 80 & 428.07 \\
25 & 133.77 & 50 & 267.55 & 75 & 401.32 \\
20 & 107.02 & 45 & 240.79 & 70 & 374.57 \\
15 & 80.26 & 40 & 214.04 & 65 & 347.81 \\
10 & 53.51 & 60 & 187.28 & 60 & 321.06 \\
\hline
\end{tabular}

Table 4.2: PMMA495 A6 Line $1 \mu \mathrm{m}$ Spacing $0.5 \mu \mathrm{m}$

\begin{tabular}{|c|c|c|c|}
\hline Pattern & $\begin{array}{c}\text { Dwell Time } \\
\mu \mathrm{s}\end{array}$ & $\begin{array}{c}\text { Dose } \\
\mu C / \mathrm{cm}^{2}\end{array}$ & $\begin{array}{c}\text { Write Time } \\
\mathrm{s}\end{array}$ \\
\hline H1 & 12 & 60.15 & 0.7 \\
$\mathrm{H} 2$ & 22 & 110.27 & 1.3 \\
$\mathrm{H} 3$ & 32 & 160.39 & 1.9 \\
$\mathrm{H} 4$ & 42 & 210.52 & 2.5 \\
H5 & 52 & 260.64 & 3.1 \\
\hline
\end{tabular}

For lines with spacing half the linewidth:

Field $13 \mu \mathrm{m} \quad$ Magnification $7570 \quad$ CC-19.96nm LS-49.99nm

For lines with spacing double the linewidth:

Field $13 \mu \mathrm{m} \quad$ Magnification $7610 \quad$ CC-20.05nm LS-49.93nm

Table 4.3: PMMA495 A6 Line $0.5 \mu \mathrm{m}$ Spacing $1 \mu \mathrm{m}$

\begin{tabular}{|c|c|c|c|}
\hline Pattern & $\begin{array}{c}\text { Dwell Time } \\
\mu \mathrm{s}\end{array}$ & $\begin{array}{c}\text { Dose } \\
\mu C / \mathrm{cm}^{2}\end{array}$ & $\begin{array}{c}\text { Write Time } \\
\mathrm{s}\end{array}$ \\
\hline D1 & 12 & 59.94 & 0.4 \\
D2 & 22 & 109.88 & 0.7 \\
D3 & 32 & 159.83 & 1.1 \\
D4 & 42 & 209.77 & 1.4 \\
D5 & 52 & 259.72 & 1.7 \\
\hline
\end{tabular}

After development, PMMA inspection under optical microscope gave the following results: 
1. Patterns were seen on sample 1 and not sample 2. There has been a placement calculation error. (Subsequently, an accurate system of ensuring sample position for writing was developed. The rotation of the center of copper grid was determined. X and Y co-ordinates that did not change on rotation of stage and still kept each sample holder in center of screen were noted as a standard. $\mathrm{X}=580, \mathrm{Y}=333$ and $\operatorname{rot}=3.0$ was determined for copper grid. The other rotations with the same $\mathrm{X}$ and $\mathrm{Y}$ are $0.5,1.75, \mathrm{Cu}, 4.25,5.5,6.75,8.0,9.25$ Fig.4.2.)

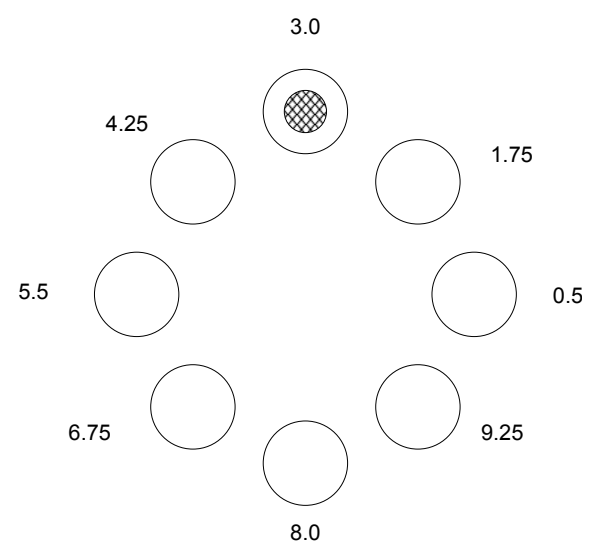

Figure 4.2: Rotation positions at $\mathrm{X}=580, \mathrm{Y}=333$

2. Star 3 was over exposed. The 3 different stars looked like pentagons. The circular ring pattern has a very thick ring and the line pattern had formed a rectangle. The patterns were written L-R top-bottom with topmost left one with highest dose.

3. In Star 2, sets 5 and 6 look better.

4. In Star 1, sets 3(133.77) and 4(107.02) were the best. Sets 5 and 6 seemed underexposed. The difference in color/brightness, especially of the line in the star pattern, is due to variation in dose Fig.4.3.

5. The line patterns, H3/4/5 were overexposed while D1/2/3 seemed underexposed. However, the lines could not be distinguished under the microscope.

6. A dot was visible between patterns i.e. between $\mathrm{H} 1$ and $\mathrm{H} 2$ and so on. So the beam wasn't off between 2 writes. 


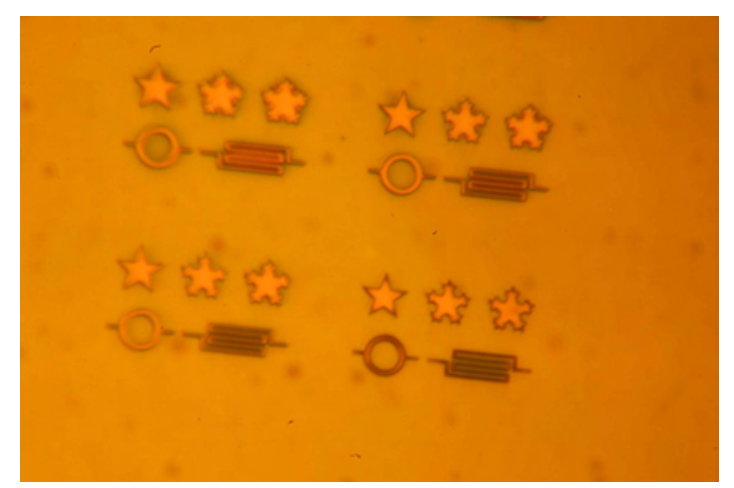

Figure 4.3: Star pattern

7. To easily view patterns the sample needs to be positioned in the SEM such that the patterns, when written, are aligned to a side.

70nm Pt was sputtered and sample was placed in SEM. Line $(1 \mu \mathrm{m})$ in star pattern is clearly seen at an exposure (133.7). In the other pictures, $0.3,0.5,0.6$ and $1 \mu \mathrm{m}$ lines are visible. 0.1 and $0.2 \mu \mathrm{m}$ lines are faintly visible.

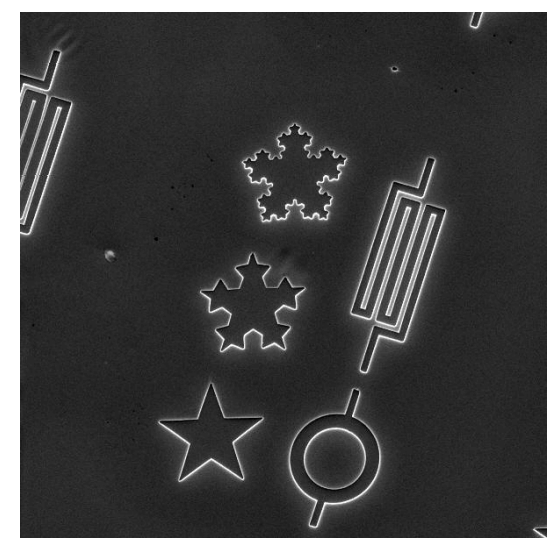

(a) Star at $133 \mu C / \mathrm{cm}^{2}$

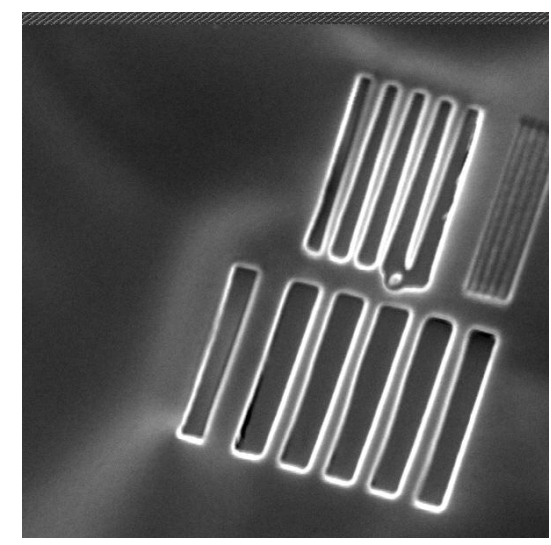

(b) Lines with half spacing

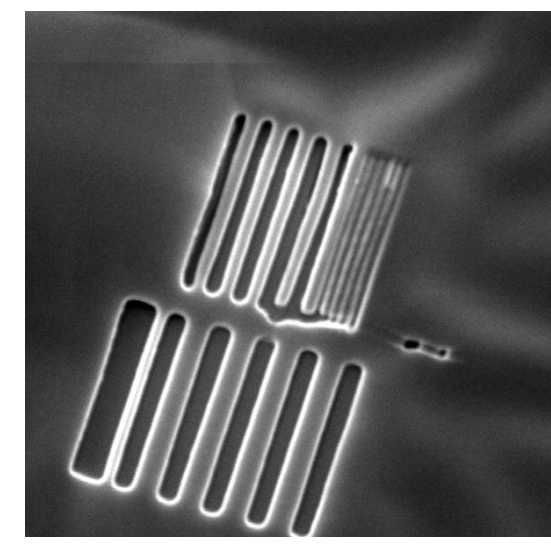

(c) Lines with double spacing

Figure 4.4: SEM results 


\subsubsection{Lines with $50 \%$ duty}

Next, files for $0.1,0.2,0.3,0.4$ and $0.5 \mu \mathrm{m}$ lines with spacing equalling linewidth were created. Also a new file for the sample file 'wheel pattern' was created. The star pattern was also written as reference.

$\mathrm{EHT}=30 \mathrm{KV}$

Working distance $=26 \mathrm{~mm} \quad$ Filament current $\mathrm{I}_{f}=2.5 \mathrm{~A} \quad$ Probe current $\mathrm{I}_{p}=49.99 \mathrm{pA}$

Table 4.4: Pattern layout M1: $0.1 \mu \mathrm{m}$ - M5: 0.5 $\mu \mathrm{m}$ Dose in parentheses

\begin{tabular}{|c|c|c|c|}
\hline Y/X & 580 & 585 & 590 \\
\hline 327 & Stars & M4(80) & M4(120) \\
332 & M1(80) & M3(120) & M5(80) \\
337 & M1(120) & M3(80) & M5(120) \\
342 & M2(80) & M2(120) & \\
\hline
\end{tabular}

Stars

Magnification $647 \quad$ CC-18.87nm LS-49.53nm

Table 4.5: Doses for star pattern

\begin{tabular}{|c|c|c|}
\hline Pattern & Dwell Time $(\mu \mathrm{s})$ & Dose $\left(\mu C / \mathrm{cm}^{2}\right)$ \\
\hline 1 & 27.098 & 145 \\
2 & 25.229 & 135 \\
3 & 23.36 & 125 \\
4 & 21.492 & 115 \\
5 & 19.623 & 105 \\
6 & 17.754 & 95 \\
\hline
\end{tabular}

Lines

Magnification $1990 \quad$ CC-19.94nm LS-49.84nm

PMMA inspection under optical microscope:

Lines were seen across some patterns. There were dots/holes in some patterns and between 
Table 4.6: Lines with 50\% duty in PMMA495 A6

\begin{tabular}{|c|c|c|c|}
\hline Feature size & Dwell Time $(\mu \mathrm{s})$ & Dose $\left(\mu C / \mathrm{cm}^{2}\right)$ & Write Time $(\mathrm{s})$ \\
\hline 0.1 & 15.898 & 80 & 20.1 \\
& 23.487 & 120 & 30 \\
0.2 & 15.898 & 80 & 20 \\
& 23.487 & 120 & 30 \\
0.3 & 15.898 & 80 & 19.9 \\
& 23.487 & 120 & 29.9 \\
0.4 & 15.898 & 80 & 20.1 \\
& 23.487 & 120 & 30.2 \\
0.5 & 15.898 & 80 & 20 \\
& 23.487 & 120 & 29.9 \\
\hline
\end{tabular}

some patterns. This is a problem of the beam blanker while writing. It is not turning the beam off even when the electrode is waiting in between writes.

70nm of Pt was sputtered. However a metal thickness of $30 \mathrm{~nm}$ or less should be used. The

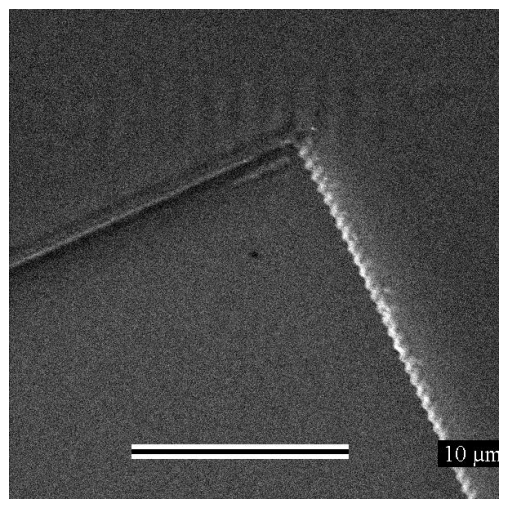

Figure 4.5: $120 \mu \mathrm{C} / \mathrm{cm}^{2}$ is over-exposure for all line sizes

thickness should be less than half the smallest feature size i.e $100 \mathrm{~nm}$.

SEM observations:

$120 \mu \mathrm{C} / \mathrm{cm}^{2}$ over-exposed lines of all feature sizes Fig.4.5. Lower doses (around $80 \mu \mathrm{C} / \mathrm{cm}^{2}$ )Fig.4.6 have to be used for the lines. For the star patterns doses of $135-145 \mu \mathrm{C} / \mathrm{cm}^{2}$ are the best whereas 95-105 $\mu \mathrm{C} / \mathrm{cm}^{2}$ causes under-exposure. 


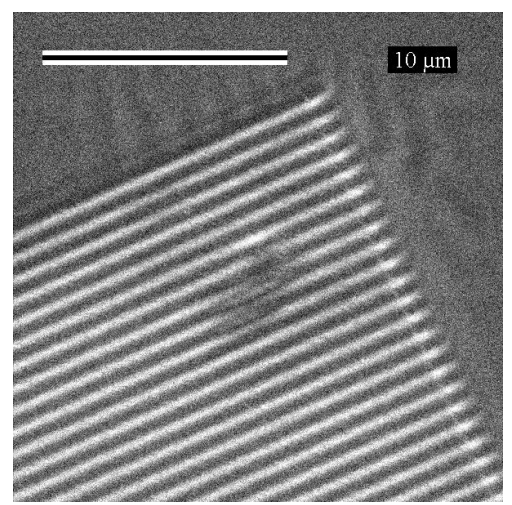

(a) $0.2 \mu \mathrm{m}$

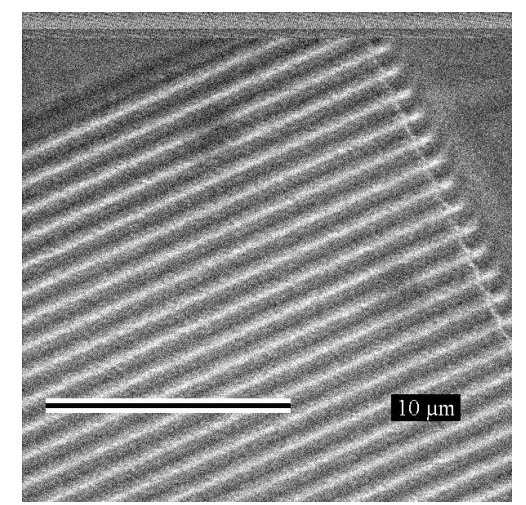

(b) $0.3 \mu \mathrm{m}$

Figure 4.6: SEM results for exposure of $80 \mu \mathrm{C} / \mathrm{cm}^{2}$

\subsubsection{Changing EHT}

The same files $(0.1,0.2,0.3,0.4$ and $0.5 \mu \mathrm{m}$ lines with equal spacing) were used with varied EHT voltage $-30 \mathrm{KV}, 35 \mathrm{KV}$ and $40 \mathrm{KV}$. The sample was sputtered with $30 \mathrm{~nm}$ Au layer. In the SEM Au film blistered as it was too thin. Images would distort very quickly Fig.4.7. Since a Pt film of $30 \mathrm{~nm}$ has been seen to work well, henceforth Pt is used for SEM viewing.

While writing, EHT was first set at 30KV and then increased to 35 and $40 \mathrm{KV}$. The Tungsten

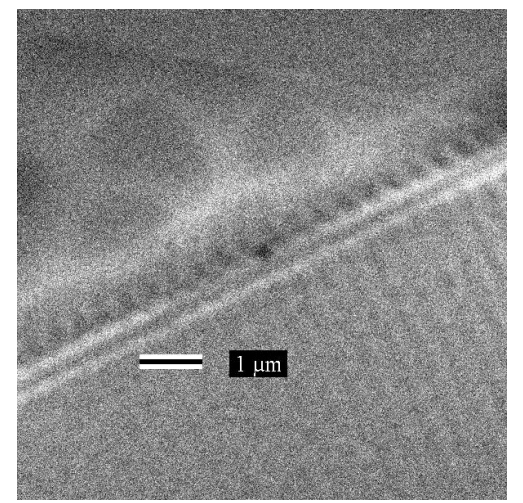

(a) $0.2 \mu \mathrm{m}$

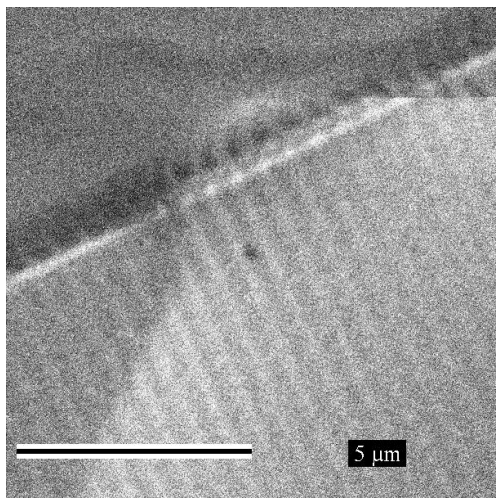

(b) $0.3 \mu \mathrm{m}$

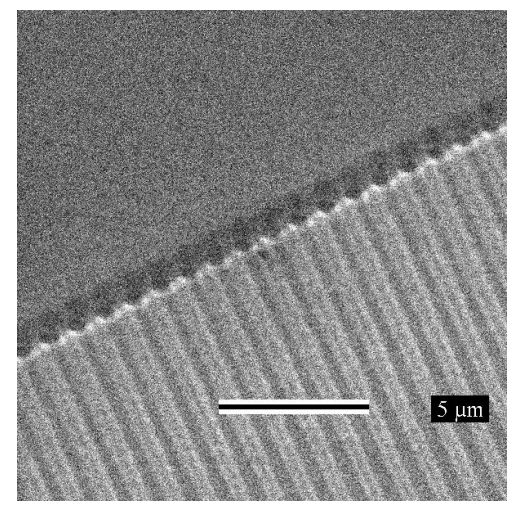

(c) $0.5 \mu \mathrm{m}$

Figure 4.7: SEM results for exposure of $80 \mu \mathrm{C} / \mathrm{cm}^{2}$ with sputtered $\mathrm{Au}$

filament burnt out as soon as the voltage was increased. Henceforth, for all the writes EHT=30KV

\footnotetext{
${ }^{2}$ http://www.jcnabity.com
} 
is used. Working at higher voltages is not possible due to system limitations. This is also the recommended working voltage ${ }^{2}$.

\subsubsection{Direct write on PMMA 495 A2}

PMMA 495 A2 when spun at 4000rpm gives a film of less than 100nm thickness. A lower thickness ensures that the e-beam exposes all the way down to the silicon. The bi-layer process of PMMA 495 A2 along with copolymer MMA-MAA EL6 is investigated later. Patterns tried were $0.1,0.2,0.3,0.4$ and $0.5 \mu \mathrm{m}$ lines with equal spacing and the stars.

$\mathrm{EHT}=30 \mathrm{KV}$

Filament current $\mathrm{I}_{f}=2.4 \mathrm{~A} \quad$ Probe current $\mathrm{I}_{p}=50.04 \mathrm{pA} \quad$ Beam blanker voltage: $60.4 \mathrm{~V}$

Table 4.7: Pattern layout in PMMA495 A2

\begin{tabular}{|c|c|c|c|}
\hline $\mathrm{Y} / \mathrm{X}$ & 575 & 580 & 585 \\
\hline 325 & Stars & $0.2-90$ & $0.2-80$ \\
330 & $0.5-90$ & $0.3-80$ & $0.1-90$ \\
335 & $0.5-80$ & $0.3-90$ & $0.1-80$ \\
340 & $0.4-90$ & $0.4-80$ & \\
\hline
\end{tabular}

The sample was then sputtered with Pt to grow a 30nm layer. Pt thickness of $30 \mathrm{~nm}$ is desired and the time of sputter was calculated from extrapolation of existing $\mathrm{Pt}$ data using polynomial equation (30nm - $0.5 \mathrm{~min})$.

Different doses gave ideal results for the different feature sizes. Grating lines were missing (due to over-exposure or excessive undercutting) at the center of some of the squares (of gratings). Observations and Conclusions:

1. For the $0.5 \mu \mathrm{m}$ lines, an exposure between $85-87 \mu \mathrm{C} / \mathrm{cm}^{2}$ should be best.

2. As seen with the decreasing feature size lower exposure of $80 \mu \mathrm{C} / \mathrm{cm}^{2}$ is better. With smaller feature sizes, the dose should be lowered.

\footnotetext{
${ }^{3}$ http://www.jcnabity.com
} 
Table 4.8: Doses for 0.1-0.5 $\mu \mathrm{m}$ in PMMA495 A2

\begin{tabular}{|c|c|c|}
\hline Feature size & $\begin{array}{c}\text { Dose } \\
\left(\mu \mathrm{C} / \mathrm{cm}^{2}\right)\end{array}$ & $\begin{array}{c}\text { Write Time } \\
(\mathrm{s})\end{array}$ \\
\hline 0.5 & 90 & 22.4 \\
& 80 & 20 \\
0.4 & 90 & 22.6 \\
& 80 & 20.1 \\
0.3 & 90 & 22.4 \\
& 80 & 19.9 \\
0.2 & 90 & 22.5 \\
0.1 & 80 & 20 \\
& 90 & 22.5 \\
& 80 & 20.1 \\
\hline
\end{tabular}

3. Distortion seen at one end (start) of the lines i.e. start of each write. This could be due to a) Beam blanker voltage or b) charging effect. A solution to the charging would be to sputter aluminum on the PMMA ${ }^{3}$.

4. $0.1 \mu \mathrm{m}$ lines were all merged up. Even an exposure of $80 \mu \mathrm{C} / \mathrm{cm}^{2}$ is high for this feature size and the trough is wider than the ridge. At many places (center) lines have been completely removed.

SEM images and results are in Appendix C. 


\subsubsection{Direct write on PMMA 495 A2 and copolymer EL6}

\subsubsection{Thin copolymer}

The bilayer process was explored. Two samples were prepared; one with PMMA and the other with PMMA and an underlying layer of the copolymer. The copolymer was spun at 4000rpm to obtain a film $100 \mathrm{~nm}$ thick. Patterns tried were $0.1,0.2,0.3,0.4$ and $0.5 \mu \mathrm{m}$ lines with equal spacing. Doses that would give better results were used for exposure.

$\mathrm{EHT}=30 \mathrm{KV}$

Filament current $\mathrm{I}_{f}=2.17 \mathrm{~A} \quad$ Probe current $\mathrm{I}_{p}=50.03 \mathrm{pA}$

Working distance $=14 \mathrm{~mm} \quad$ Beam blanker voltage $=22 \mathrm{~V}$

Table 4.9: Positions and doses of patterns on PMMA495 A2

\begin{tabular}{|c|c|c|c|c|c|}
\hline Y/X & 487 & 482 & 477 & 472 & 467 \\
\hline 297 & $0.1-70$ & $0.5-87$ & $0.5-87$ & $0.5-87$ & $0.1-70$ \\
302 & $0.1-65$ & $0.4-80$ & $0.4-80$ & $0.4-80$ & $0.1-65$ \\
307 & $0.1-60$ & $0.3-77$ & $0.3-77$ & $0.3-77$ & $0.1-60$ \\
312 & $0.1-55$ & $0.2-75$ & $0.2-75$ & $0.2-75$ & $0.1-55$ \\
317 & $0.1-50$ & & & & $0.1-50$ \\
\hline
\end{tabular}

$\mathrm{X}$-Y: linewidth $(\mu \mathrm{m})$-dose $\left(\mu \mathrm{C} / \mathrm{cm}^{2}\right)$

Patterns on the copolymer sample were written similarly.

Table 4.10: Exposures for different line sizes in PMMA495 A2

\begin{tabular}{|c|c|c|}
\hline Feature size $(\mu \mathrm{m})$ & Dose $\left(\mu C / \mathrm{cm}^{2}\right)$ & Write Time $(\mathrm{s})$ \\
\hline 0.5 & 87 & 22.4 \\
0.4 & 80 & 22.6 \\
0.3 & 77 & 19.2 \\
0.2 & 75 & 18.7 \\
0.1 & 70 & 17.6 \\
& 65 & 16.3 \\
& 60 & 15.1 \\
& 55 & 13.8 \\
& 50 & 12.6 \\
\hline
\end{tabular}


All patterns were visible under the optical microscope. However a dot was seen in the center of $0.1 \mu \mathrm{m}$ lines. The beam blanker voltage of $22 \mathrm{~V}$ may be too low to sufficiently move the beam off. When viewed under white light: a) On Sample 1 change in color is distinct only for 0.5 and $0.4 \mu \mathrm{m}$ lines. b) On Sample 2 distinct colors were seen for 0.5, 0.4 and $0.3 \mu \mathrm{m}$ lines. However, only the outlines of squares were seen.

Samples were then sputtered with Pt to grow a 30nm layer.

SEM images and results are in Appendix C.

SEM observations and conclusions:

1. There were no lines on the copolymer+PMMA495 A2 sample. Squares were seen. This is in conjunction with what was seen under the optical microscope. From the images, it seems as though all the copolymer had got developed (over-exposure) and hence the PMMA495 A2 had lifted off.

2. All lines from $0.5-0.2 \mu \mathrm{m}$ were seen on the PMMA495 A2 sample. The $0.1 \mu \mathrm{m}$ lines are still indistinct. The lines are merged in the center of the square and at the start of the write. With the lower exposures, lines seem to have been attempted. But the e-beam energy was insufficient to expose the resist till the silicon.

3. The sample was tilted to $45^{\circ}$. But the sidewalls at the cleaved edge of the pattern could not be seen or measured. Measurement of groove depth was not possible. A better way of cleaving the sample must be devised to view sidewalls of features.

4. The distortion seen at the start of the write process in the previous tests is no longer there. It seems to be the beam blanker voltage that caused it the last time (61V).

To estimate the thickness of the copolymer, it was spun onto the silicon sample and a line was scratched. Thickness was measured at different places on the line (Alpha Step) and was found to be about $210 \mathrm{~nm}$. A higher speed or increase in the time of spin should give a lower thickness. $210 \mathrm{~nm}$ does not match the thickness from the speed-thickness curve. This could be because the 
copolymer was taken from the cold storage and its viscosity must have been higher than that at room temperature. Even the PMMA495 A2 was a thicker film. Further investigation revealed that this was due to increase in humidity (in the lab). Once it was lowered, appropriate PMMA495 A2/copolymer thicknesses were attained. Their thickness is critical in order to have appropriate exposures.

\subsubsection{Single pass of beam and use of metals}

To avoid distortion at the start of the lines, the use of a single pass of the beam was suggested ${ }^{3}$. Patterns tried were $0.1,0.2,0.3,0.4$ and $0.5 \mu \mathrm{m}$ lines with equal spacing (same as before). Patterns using single pass of the beam were also written for the above feature sizes. As the center-to-center distance should be 1/4-1/2 the desired line width, the line spacing was selected such that the lines and spaces are equal in size ( $\mathrm{C}-\mathrm{C}$ of $0.2,0.4,0.6,0.8$ and 1$)$. The programs use the 'line draw' command as opposed to 'polyfill' in the previous programs.

Under the optical microscope, all patterns were seen, but no lines were visible even under x50 lens. There is a possibility that 'Auto-brightness' was left 'on' when writing and hence the lines may not be written as the intensity may have been too high. This would cause an error while writing as the SEM would try to auto-adjust while the pattern is to be written at a fixed dose.

\section{Use of chrome in a lift-off process:}

Patterns that were written on PMMA495 A2 were then used in chrome lift-off. Chrome was evaporated on the patterned resist. It was thicker than desired ( $400 \mathrm{~nm}$ on Alpha step) whereas 50nm was needed. Lift-off was attempted with PMMA495 A2 strip.

PMMA strip: 30 min. soak; Sonication: 1min.; Acetone: 5 min.; Methanol: 5 min.; $\mathrm{N}_{2}$ dry. Along with the chrome all the PMMA was lifted off. In the place of lines there were metal squares. Under the optical microscope, the edges looked jagged. Fig.4.8 shows a square as seen when backed out. The lines were not seen. Either the lines were over-exposed resulting in squares or due to the thick chrome the patterned resist could not lift-off the chrome. 


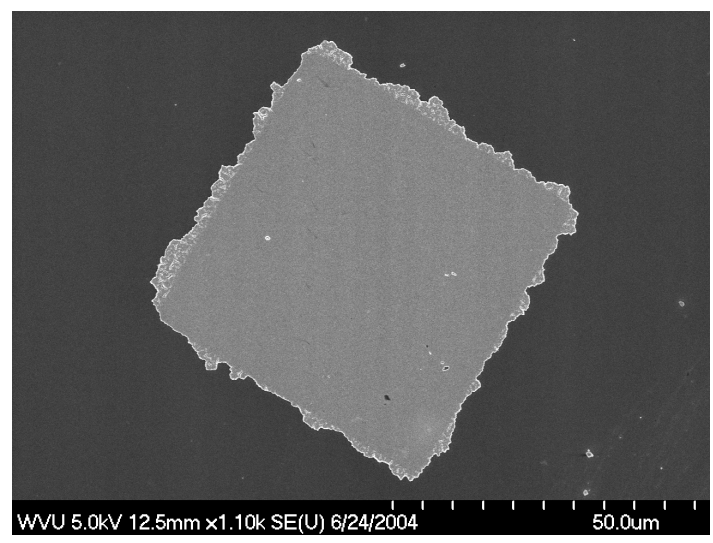

Figure 4.8: $50 \mu \mathrm{m}$ square with no lines

\section{Use of Aluminum:}

Patterns written can be distorted when the substrate is not too conductive. Since charging of a substrate may cause pattern distortion, often a $10 \mathrm{~nm} \mathrm{Al} \mathrm{layer} \mathrm{is} \mathrm{sputtered} \mathrm{on} \mathrm{top} \mathrm{of} \mathrm{the} \mathrm{resist}$ before writing. $\mathrm{Al}$ is removed by $\mathrm{NaOH}$ or $\mathrm{KOH}$ after the writing and before developing the resist.

A thin layer of aluminum was sputtered for $0.3 \mathrm{~min}(11.5 \mathrm{~nm})$ on a PMMA layer of $80-90 \mathrm{~nm}$. After e-beam exposure the Aluminum was etched using $\mathrm{NaOH}$. On development in MIBK/IPA, all the PMMA was removed and no features were left.

This could be because $\mathrm{NaOH}$ was used directly for etch while only a few pellets of $\mathrm{NaOH}$ in $100 \mathrm{ml}$ of DI water should be used to etch for about 30 seconds. In this case, $\mathrm{NaOH}$ may have permeated the PMMA and chemically interacted with it.

Another aluminum etchant is a very weak HF solution (50\% by weight diluted 100:1). The $\mathrm{Al}$ is etched in a few seconds and is then rinsed in DI water and then IPA.

Aluminum can be etched using the Type A etchant from Transene [25]. References show $\mathrm{NaOH}$ used to remove/develop photoresist, hence it may remove PMMA too.

Diluted $\mathrm{NaOH}$ (less than 1\%) and diluted Transene etchant were tried. After Al removal the PMMA still came off. When a sample of PMMA + Al were put first in the developer (without $\mathrm{Al}$ etch), both $\mathrm{Al}$ and PMMA remained. $\mathrm{Al}$ was then etched off 2 samples in each etchant and 
PMMA was measured and found to be $90 \mathrm{~nm}$. After both etches, PMMA color changed a bit indicating some chemical reaction. On treatment with the developer the resist came off.

To understand why the PMMA comes off after Al etchant treatment, an unexposed PMMA sample was immersed in an $\mathrm{Al}$ etchant $(\mathrm{NaOH}) 30$ s. and $\mathrm{DI}$ water 30s. and then developed in MIBK-IPA 1:1 30s and then in 1:3 for 30s. The PMMA did NOT dissolve in the developer. Another sample with PMMA and Al was treated the same without e-beam exposure. This time all the PMMA was removed on development.The use of an Acid ( $\mathrm{HCl}$ or acetic) etch instead of an alkali etch was recommended ${ }^{4}$.

$20 \mathrm{ml} \mathrm{HCl}+30 \mathrm{ml}$ De-I water took a long time (>20mins) to etch Al. After Al removal the PMMA dissolved in developer. $\mathrm{Al}$ etchant $\left(\mathrm{H}_{3} \mathrm{PO}_{4}+\mathrm{HNO}_{3}+\mathrm{CH}_{3} \mathrm{COOH}\right)$ diluted in De-I (1:1) was also tried. In this case also the PMMA dissolved in developer after $\mathrm{Al}$ etch (30s).

The removal of PMMA when $\mathrm{Al}$ is present suggests a chemical reaction between both especially on etching Al causing PMMA to be removed on development. Detailed study is needed before using the $\mathrm{Al}$ layer in direct write.

\subsubsection{Dose optimization}

As the patterns on the previous attempts were not observed under the SEM, the same were repeated without aluminum and chrome. It is necessary to optimize the dose before attempting lift-off. Once the optimum dose was ascertained, metal was deposited and then lifted-off. Two samples (Sample 1 PMMA, Sample 2 PMMA + copolymer)were written by e-beam.

\section{$\mathrm{EHT}=30 \mathrm{KV}$}

Filament current $\mathrm{I}_{f}=2.43 \mathrm{~A} \quad$ Probe current $\mathrm{I}_{p}=49.99 \mathrm{pA}$

Working distance $15 \mathrm{~mm} \quad$ Beam blanker voltage: $40 \mathrm{~V}$

$\mathrm{X}$-Y: linewidth $(\mu \mathrm{m})$-dose $\left(\mu C / \mathrm{cm}^{2}\right)$

Though all the patterns were seen under the optical microscope, lines were visible on sample 1 for only $0.3-0.5 \mu \mathrm{m}$ features. For patterns written with single pass of beam, lines were visible

\footnotetext{
${ }^{4}$ Microchem
} 
Table 4.11: Layout of patterns on Sample 1

\begin{tabular}{|c|c|c|c|c|c|}
\hline Y/X & 570 & 575 & 580 & 585 & 590 \\
\hline 320 & $0.5-82$ & $0.5-120$ & $0.5-100$ & $0.5-80$ & $0.5-82$ \\
325 & $0.4-77$ & $0.4-120$ & $0.4-100$ & $0.4-80$ & $0.4-77$ \\
330 & $0.3-70$ & $0.3-120$ & $0.3-100$ & $0.3-80$ & $0.3-70$ \\
335 & $0.2-65$ & $0.2-100$ & $0.2-80$ & $0.2-60$ & $0.2-65$ \\
340 & $0.1-75$ & $0.2-80$ & $0.2-60$ & $0.2-40$ & $0.1-75$ \\
345 & $0.1-65$ & & & & $0.1-65$ \\
\hline
\end{tabular}

Table 4.12: Doses for serpentine sweep of beam

\begin{tabular}{|c|c|c|}
\hline Feature size $(\mu \mathrm{m})$ & Dose $\left(\mu C / \mathrm{cm}^{2}\right)$ & Write Time $(\mathrm{s})$ \\
\hline 0.5 & 82 & 20.5 \\
0.4 & 77 & 19.4 \\
0.3 & 70 & 17.4 \\
0.2 & 65 & 16.3 \\
0.1 & 75 & 18.8 \\
& 65 & 16.3 \\
\hline
\end{tabular}

at $100 \mathrm{x}$ magnification, only at the start/end of $0.5 \mu \mathrm{m}$ feature. On sample 2 , single pass patterns were overexposed.

Pt was sputtered for $0.5 \mathrm{~min}$ to grow a $30 \mathrm{~nm}$ layer.

SEM images are in Appendix C. On the PMMA sample, the serpentine sweep patterns are under-exposed. Only the $0.3 \mu \mathrm{m}$ lines written with single pass of the beam were completely formed. However, the line spacing varied. All the single pass patterns on Sample 2 looked similar to the pattern in Fig.6.9 in Appendix C. No lines were seen and the whole square was exposed. On the other patterns (serpentine sweep), only the center hole could be seen on every pattern and the outlining square was barely discernible. Since the PMMA+copolymer layer was really thick (PMMA $\sim 100 \mathrm{~nm}$ and copolymer $\sim 400 \mathrm{~nm}$ ), the lines may not have gone down through the silicon and may be just on the surface.

Conclusions: PMMA thickness has to be accurate (70nm). For the serpentine sweep patterns, best dose for $0.5 \mu \mathrm{m}$ lines is $85 \mu \mathrm{C} / \mathrm{cm}^{2}, 0.4-80$ and $0.3-75$. For single pass of beam, $0.5 / 0.4$ 
Table 4.13: Doses for single pass of beam

\begin{tabular}{|c|c|c|c|}
\hline Feature size & Magnification & Dose $\left(\mu C / \mathrm{cm}^{2}\right)$ & Write Time $(\mathrm{s})$ \\
\hline 0.5 & 1640 & 120 & 42.6 \\
& & 100 & 35.5 \\
0.4 & \multirow{3}{*}{1640} & 80 & 28.4 \\
& & 120 & 34.5 \\
& & 100 & 28.8 \\
0.3 & \multirow{3}{*}{1580} & 80 & 23 \\
& & 120 & 39 \\
0.2 & \multirow{3}{*}{1620} & 100 & 32.5 \\
& & 80 & 26 \\
0.1 & \multirow{3}{*}{1590} & 80 & 36 \\
& & 60 & 28.8 \\
& & 80 & 21.6 \\
& & 60 & 29.1 \\
& & 40 & 21.9 \\
& & & \\
\hline
\end{tabular}

lines need $<100 \mu \mathrm{C} / \mathrm{cm}^{2}$ and $0.3-100 \mu \mathrm{C} / \mathrm{cm}^{2}$. Single pass of beam gives better results than serpentine sweep.

\subsubsection{Thick copolymer}

Various references show that the copolymer should be much thicker than the PMMA layer. In a thin copolymer, the backscattered electrons could undercut adjacent lines in the copolymer so as to expose it all, which on development would lift off all the PMMA. A thicker copolymer $400 \mathrm{~nm}$ is used henceforth. Since the gratings are to be etched in borofloat the copolymer-resist layer was spun on a borofloat sample. Also since a beam blanker voltage of $60 \mathrm{~V}$ gave the best write results and effectively turned off the beam, $60 \mathrm{~V}$ is used in the following write experiments.

None of the patterns written on borofloat were visible after development. Since borofloat is a transparent, dielectric material as opposed to silicon which is opaque and conductive, the method of writing and the doses will have to be optimized for borofloat. Due to constraints on resources and time it was decided that the bilayer process for silicon would be optimized. Only $0.5 \mu \mathrm{m}$ 
lines were written on the bilayer sample in order to optimize the dose for one feature size. To also improve the direct-write doses on PMMA, a silicon sample with PMMA was patterned with changed doses as concluded from previous experiments.

$\mathrm{EHT}=30 \mathrm{KV}$

Filament current $\mathrm{I}_{f}=2.66 \mathrm{~A} \quad$ Probe current $\mathrm{I}_{p}=50.03 \mathrm{pA}$

Working distance $16 \mathrm{~mm} \quad$ Beam blanker voltage: $60 \mathrm{~V}$

Table 4.14: Modified doses for serpentine sweep of beam on PMMA A2

\begin{tabular}{|c|c|c|}
\hline Feature size $(\mu \mathrm{m})$ & Dose $\left(\mu C / \mathrm{cm}^{2}\right)$ & Write Time $(\mathrm{s})$ \\
\hline 0.5 & 85 & 21.2 \\
0.4 & 80 & 20.1 \\
0.3 & 77 & 19.2 \\
0.2 & 75 & 18.7 \\
0.1 & 70 & 17.6 \\
\hline
\end{tabular}

Table 4.15: Modified doses for single sweep of beam on PMMA A2

\begin{tabular}{|c|c|c|c|}
\hline Feature size & Magnification & Dose $\left(\mu C / \mathrm{cm}^{2}\right)$ & Write Time $(\mathrm{s})$ \\
\hline 0.5 & 1640 & 100 & 35.6 \\
& & 90 & 32 \\
0.4 & \multirow{3}{*}{1640} & 80 & 28.4 \\
& & 100 & 28.8 \\
0.3 & \multirow{3}{*}{1580} & 90 & 26 \\
& & 80 & 23.1 \\
& & 100 & 32.5 \\
& & 90 & 29.2 \\
\hline
\end{tabular}

Observations under Optical Microscope:

All the patterns were seen on both the samples. On sample 2, lines were seen for the pattern written with a single pass of the beam with a dose of $70 \mu \mathrm{C} / \mathrm{cm}^{2}$ Fig.4.9. The lines diffracted red light when the microscope (white) light was normally incident on it. 
Table 4.16: Doses for serpentine sweep of beam on bilayer

\begin{tabular}{|c|c|}
\hline Dose $\left(\mu C / \mathrm{cm}^{2}\right)$ & Write Time $(\mathrm{s})$ \\
\hline 120 & 29.9 \\
110 & 27.4 \\
105 & 26.2 \\
100 & 24.9 \\
90 & 22.4 \\
\hline
\end{tabular}

Table 4.17: Doses for single sweep of beam on bilayer

\begin{tabular}{|c|c|c|c|}
\hline Dose $\left(\mu C / \mathrm{cm}^{2}\right)$ & Write Time $(\mathrm{s})$ & Dose $\left(\mu C / \mathrm{cm}^{2}\right)$ & Write Time $(\mathrm{s})$ \\
\hline 150 & 53.3 & 100 & 35.6 \\
140 & 49.8 & 90 & 32 \\
130 & 46.2 & 80 & 28.4 \\
120 & 42.7 & 70 & 24.9 \\
110 & 39.1 & & \\
\hline
\end{tabular}
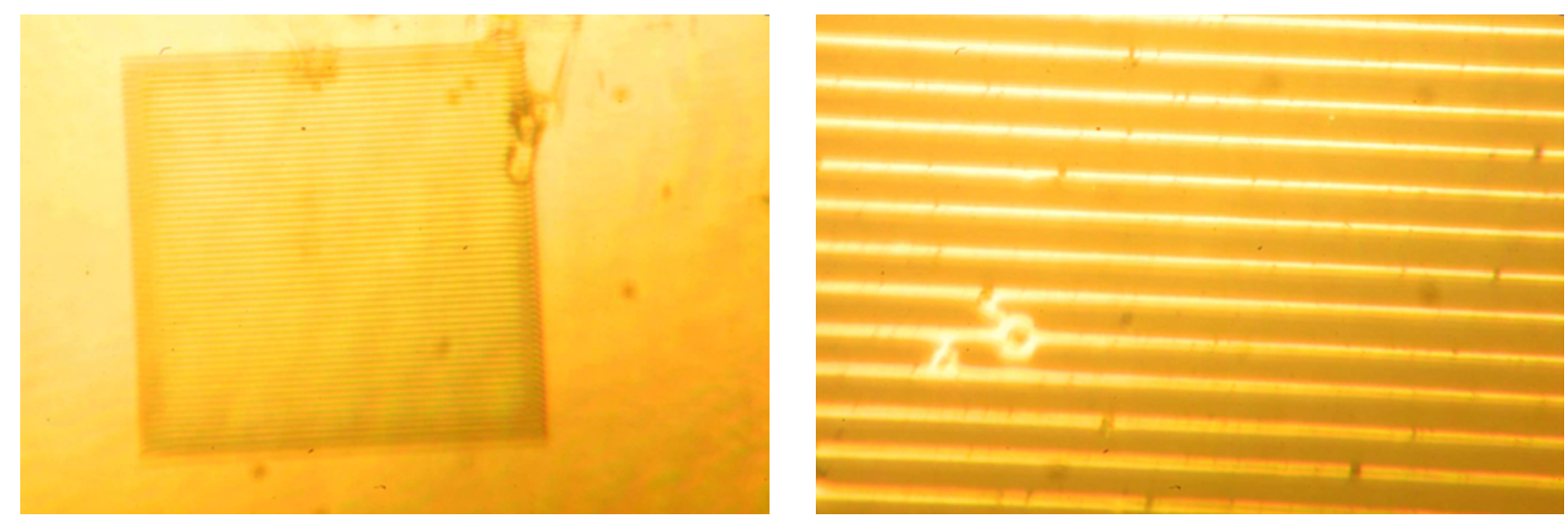

Figure 4.9: $0.5 \mu \mathrm{m}$ lines at exposure of $70 \mu \mathrm{C} / \mathrm{cm}^{2}$ as seen under the optical microscope

\section{SEM results:}

Under the SEM it was difficult to find the patterns on the PMMA. Grounding the sample with conducting tape was also tried. It has been observed that if the resist layer is very thin or if the there are no lines (i.e. either over-exposed or under-exposed and only a square outline is visible in the optical microscope), these patterns are hard to find in the SEM. The bilayer being thicker and the lines distinct for one exposure, they were seen in the SEM. 

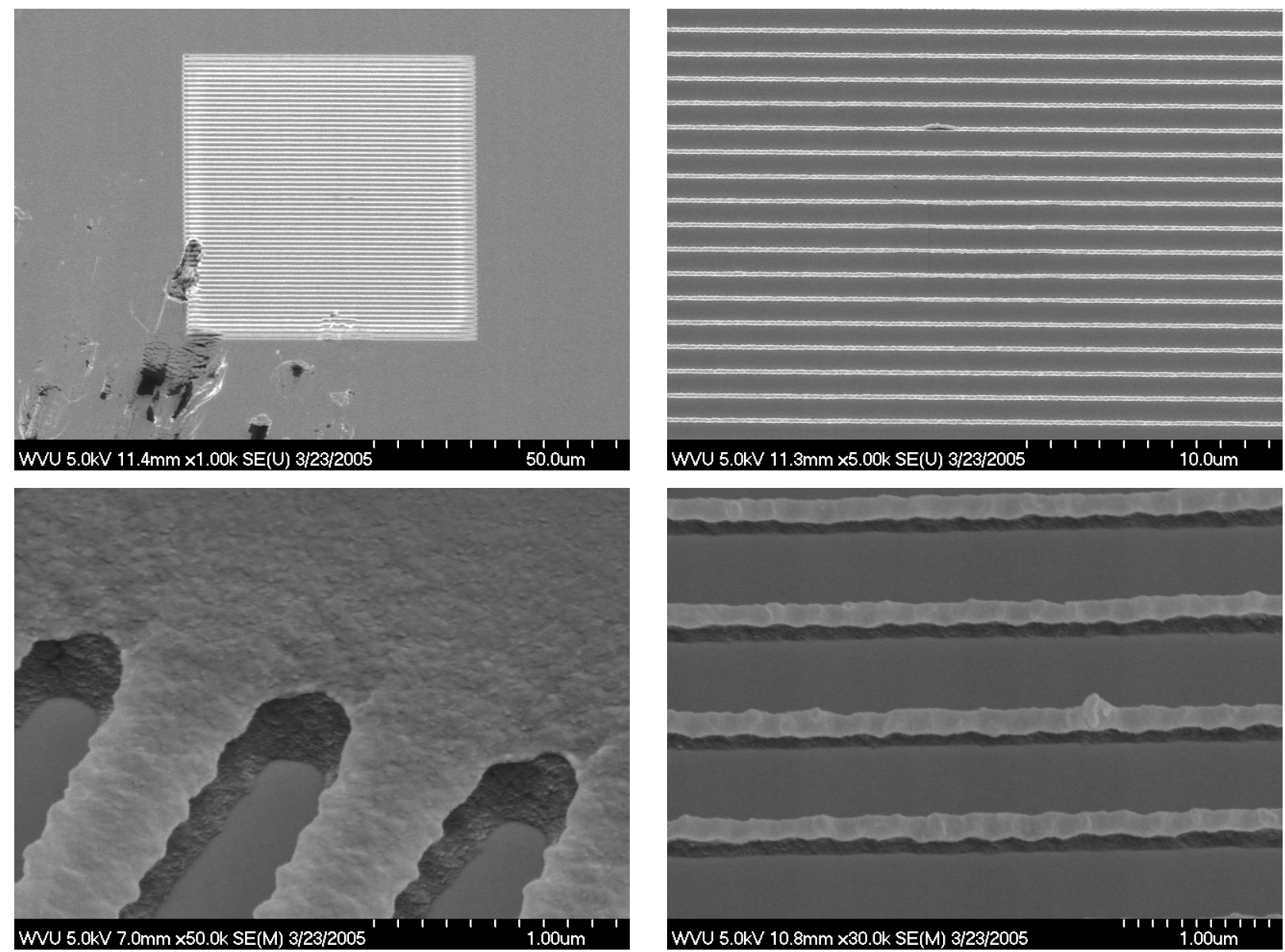

Figure 4.10: SEM images of $0.5 \mu \mathrm{m}$ lines in resist bilayer written at an exposure of $70 \mu \mathrm{C} / \mathrm{cm}^{2}$

The lines thin at the center of the pattern. The spacing between the lines increases towards the center. Freestanding lines about $100 \mathrm{~nm}$ wide have been obtained. The copolymer is undercut as can be seen at the end of the lines. The exposure and development are appropriate and the silicon can be seen in the developed areas. As platinum was sputtered to view the samples in the SEM, a lift-off was then attempted and the silicon dry etched with SF6 using platinum as the mask. The etch results are discussed in section 4.3.

Since for RIE of borofloat, chrome is to be deposited and lifted-off, the bilayer is essential. Further optimization for $0.5 \mu \mathrm{m}$ lines and dose estimation for the smaller features were done. 
$\mathrm{EHT}=30 \mathrm{KV}$

Filament current $\mathrm{I}_{f}=2.49 \mathrm{~A} \quad$ Probe current $\mathrm{I}_{p}=49.99 \mathrm{pA}$

Working distance $16 \mathrm{~mm} \quad$ Beam blanker voltage: $60.1 \mathrm{~V}$

Table 4.18: Doses for 0.4 and $0.3 \mu \mathrm{m}$ lines using serpentine sweep of beam

\begin{tabular}{|c|c|c|}
\hline Feature Size & 0.4 & 0.3 \\
\hline Dose $\left(\mu C / \mathrm{cm}^{2}\right)$ & Write Time $(\mathrm{s})$ & Write Time $(\mathrm{s})$ \\
\hline 60 & 15.1 & 15 \\
50 & 12.6 & 12.5 \\
40 & 10.1 & 10 \\
30 & 7.6 & 7.5 \\
20 & 5.1 & 5 \\
\hline
\end{tabular}

Table 4.19: Doses for 0.2-0.4 $\mu \mathrm{m}$ lines using single pass of beam

\begin{tabular}{|c|c|c|c|}
\hline Feature size & Magnification & Dose $\left(\mu C / \mathrm{cm}^{2}\right)$ & Write Time $(\mathrm{s})$ \\
\hline 0.4 & 1640 & 60 & 17.3 \\
& & 50 & 14.4 \\
0.3 & \multirow{3}{*}{1580} & 40 & 11.6 \\
& & 55 & 17.9 \\
0.2 & \multirow{3}{*}{1620} & 45 & 14.6 \\
& & 35 & 11.4 \\
& & 50 & 18.1 \\
& & 90 & 16.3 \\
& & 80 & 14.5 \\
\hline
\end{tabular}

Many patterns were found to diffract under the optical microscope. The best SEM images were for 0.4 and $0.3 \mu \mathrm{m}$, at $60 \mu \mathrm{C} / \mathrm{cm}^{2}$ with a single sweep of the beam. The lines were of equal width and equally spaced.

The serpentine sweep for the same feature sizes did not go down to the silicon. This is evident from the blurred lines Fig.4.12. It was also seen that in many patterns the lines had not been formed along the whole length and did not go all the way down to the silicon. However, due to the periodic exposures, the semi-patterned areas also diffract.

From the above exposures and SEM images, it was seen that even the slightest dose difference or PMMA thickness variation, could change the developed pattern. For $0.5 \mu \mathrm{m}, 70 \mu \mathrm{C} / \mathrm{cm}^{2}$ gave 
Table 4.20: Modified doses for 0.3-0.5 $\mu \mathrm{m}$ lines using serpentine sweep of beam

\begin{tabular}{|c|c|c|}
\hline Feature size $(\mu \mathrm{m})$ & Dose $\left(\mu C / \mathrm{cm}^{2}\right)$ & Write Time $(\mathrm{s})$ \\
\hline 0.5 & 75 & 18.7 \\
& 70 & 17.5 \\
& 65 & 16.2 \\
& 60 & 15 \\
0.4 & 55 & 13.7 \\
& 70 & 17.6 \\
0.3 & 65 & 16.4 \\
& 55 & 13.8 \\
& 65 & 16.2 \\
& 55 & 13.7 \\
\hline
\end{tabular}
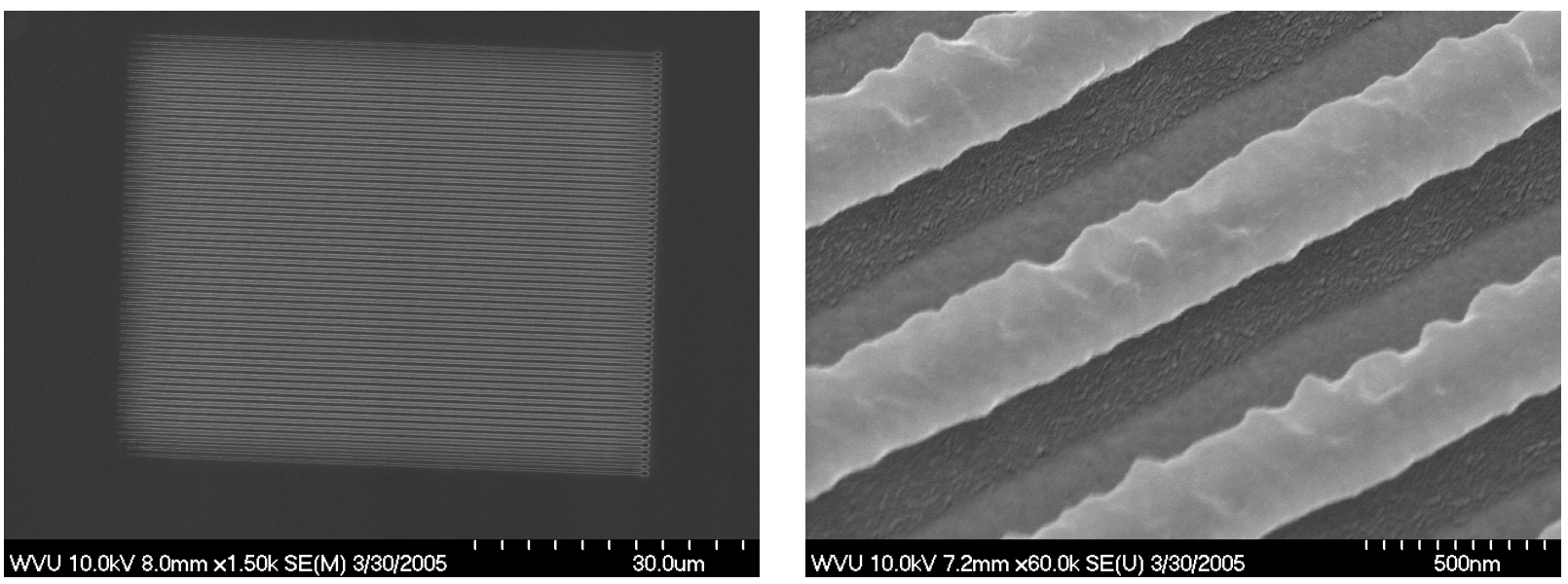

Figure 4.11: $0.4 \mu \mathrm{m}$ lines at exposure of $60 \mu \mathrm{C} / \mathrm{cm}^{2}$

the best lines whereas for both 0.4 and $0.3 \mu \mathrm{m}, 60 \mu \mathrm{C} / \mathrm{cm}^{2}$ gave well defined lines. When using serpentine sweep of the beam, higher doses are needed for the same feature sizes. 
Table 4.21: Modified doses for 0.2-0.5 $\mu \mathrm{m}$ lines using single sweep of beam

\begin{tabular}{|c|c|c|c|}
\hline Feature size & Magnification & Dose $\left(\mu C / \mathrm{cm}^{2}\right)$ & Write Time $(\mathrm{s})$ \\
\hline 0.5 & 1640 & 70 & 24.9 \\
& & 65 & 23.1 \\
0.4 & \multirow{3}{*}{1640} & 60 & 21.3 \\
& & 70 & 20.2 \\
0.3 & \multirow{3}{*}{1580} & 65 & 18.8 \\
& & 55 & 15.9 \\
& & 70 & 22.8 \\
& \multirow{3}{*}{0.2} & 65 & 21.1 \\
& \multirow{3}{*}{1620} & 55 & 17.9 \\
& & 70 & 25.3 \\
& & 65 & 23.5 \\
& & 60 & 21.7 \\
\hline
\end{tabular}

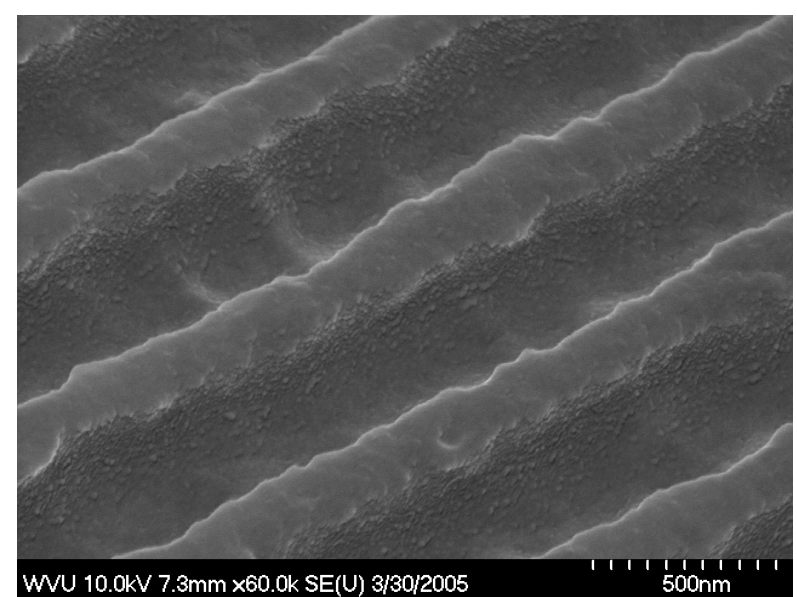

(a) $0.3 \mu \mathrm{m}$ lines written with serpentine sweep

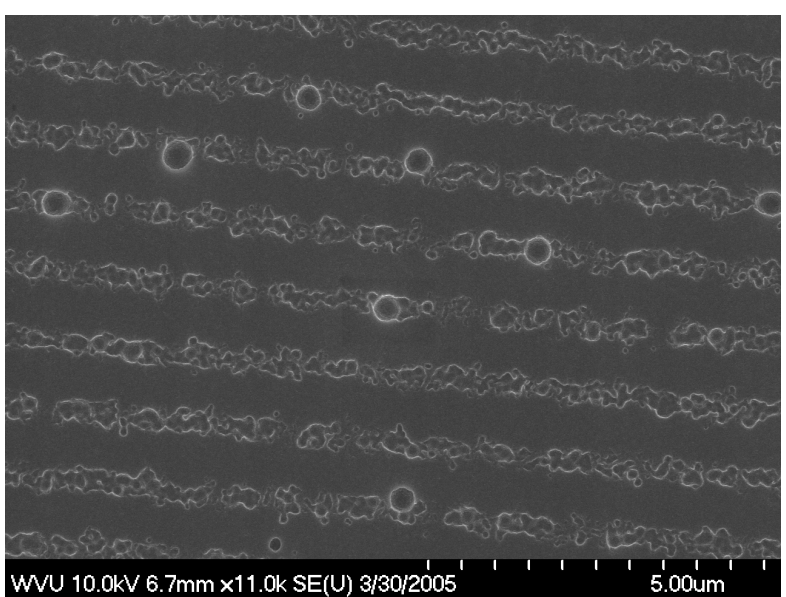

(b) Semi-patterned periodic structures that diffract

Figure 4.12: Some features at $60 \mu \mathrm{C} / \mathrm{cm}^{2}$ 


\subsection{RIE}

Different RIE recipes were tried out. The gas chemistries, flows, power and pressures were varied. To test the recipes borofloat samples were patterned (microlens structures) by photolithography. Initially photoresist was used as the masking material. Later, chrome was sputtered/evaporated to be used as the mask instead of resist as that would be needed when PMMA is used for e-beam lithography (PMMA will not withstand the plasma etch).

Creating test samples: Borofloat was coated with AZ5214 and patterned using photolithography.

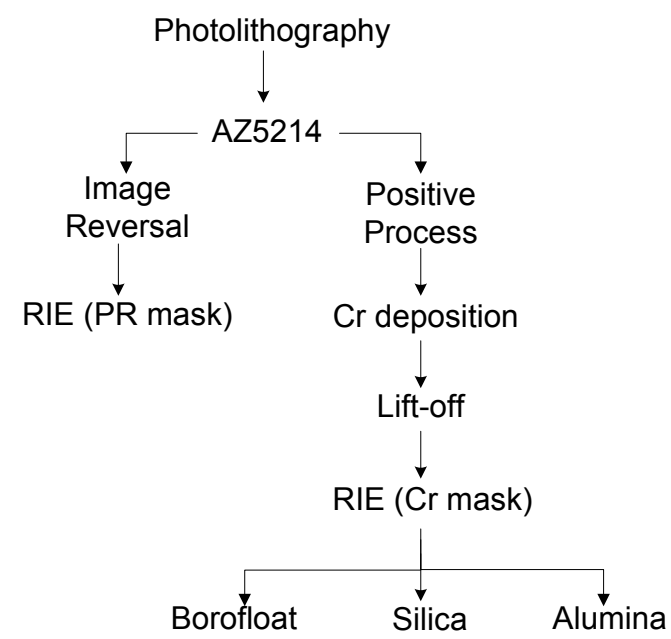

Figure 4.13: Process development for RIE samples

The various processes developed and the materials etched are shown in Fig.4.13. AZ5214 is an image reversal resist. Using it in IR mode, resist was used as the mask for RIE. The process steps are shown in Fig.4.14 (process steps in Appendix 1). Since with e-beam lithography, a metal mask would be required, AZ5214 was then used as a positive resist. Chrome was sputtered/ evaporated on the patterned resist and then lifted-off using acetone. RIE results with chrome as a mask showed better sidewalls. Sputtered chrome was grainier than chrome deposited by evaporation as seen in SEM. 


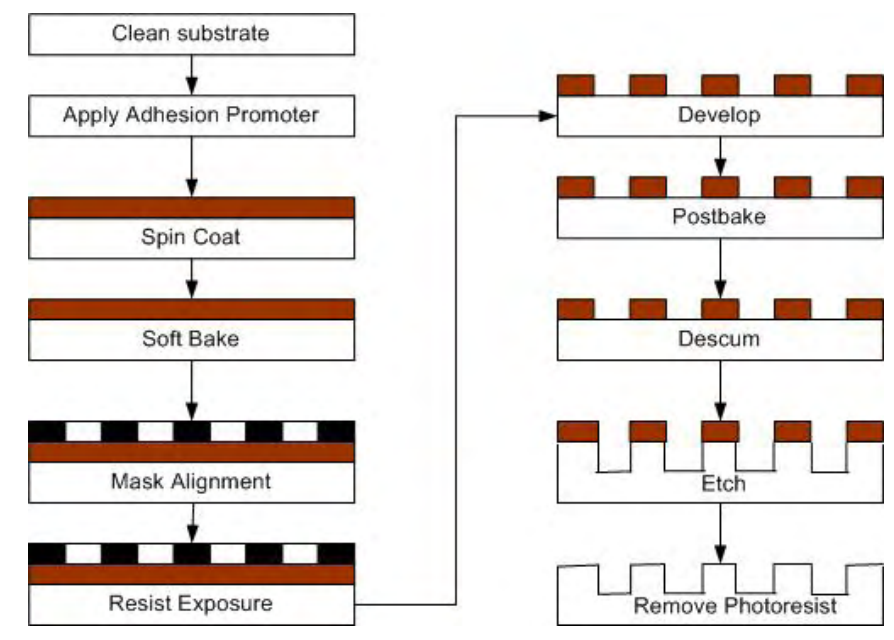

Figure 4.14: Photoresist fabrication of gratings

\subsection{1 $\mathrm{CF}_{4}$ etch}

We began with a $\mathrm{CF}_{4} / \mathrm{O}_{2}$ recipe with resist as the mask. This has been demonstrated on Pyrex glass [25]. Flow rate is measured in standard cubic centimeters per minute, pressure is in millitorr, power between cathode and anode is measured in watts and etch time is entered in minutes and seconds.

\begin{tabular}{|c|c|c|c|c|c|}
\hline Gases & Flow Rate(sccm) & Pressure (mT) & RF Power (W) & ICP Power (W) & Time \\
\hline $\mathrm{CF}_{4} / \mathrm{O}_{2}$ & $25 / 5$ & 60 & 100 & 0 & $16 \mathrm{~m} \mathrm{52s}$ \\
\hline
\end{tabular}

The above recipe should have etched Pyrex to a depth of $520 \mathrm{~nm}$ (etch rate of $31 \mathrm{~nm} / \mathrm{min}$ [25]). However, the etch depth measured by the Alpha Step profilometer gave 314nm indicating a slower etch rate. This can be attributed to the difference in the RIE systems used. The sloped side-wall could be due to under-exposure or over-development of the resist in the lithography step. The non-volatile compounds are not etched as is clearly seen in Fig.4.15(a). A more physical etch is needed to remove them. The marked line at the bottom of the side-wall could be due to microtrenching when electrons ricochet off the opposite side-wall and strike the edge of the feature being etched [5] Fig.4.15(a).

To attain a more physical etch, lower chamber pressures and gas flows were found necessary 


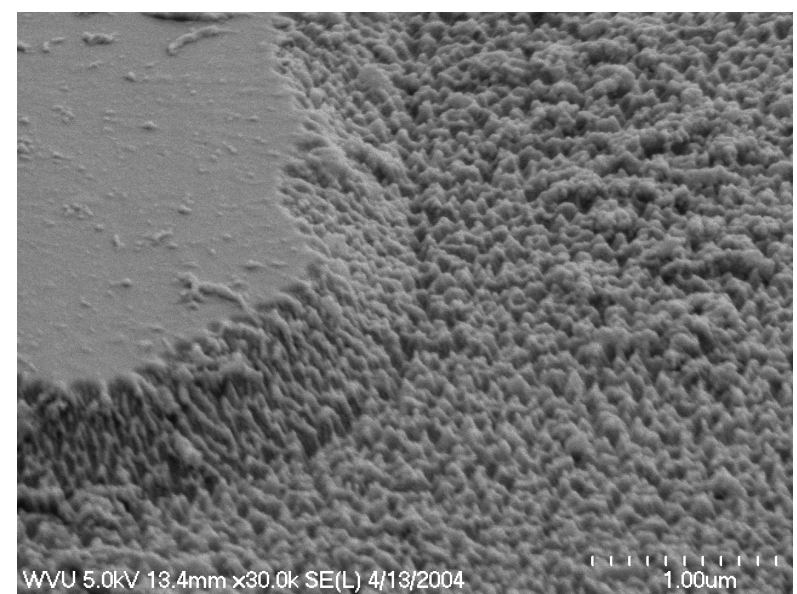

(a) $\mathrm{CF}_{4} / \mathrm{O}_{2}$ etch result

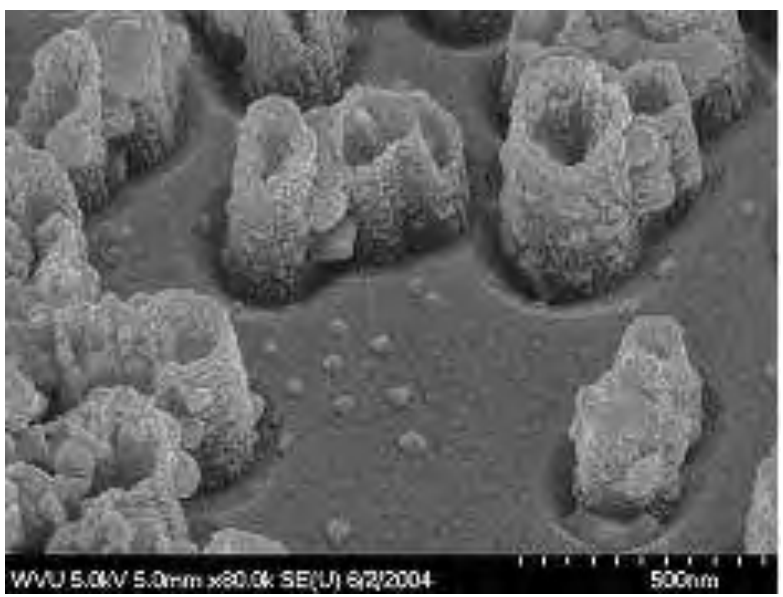

(b) $C F_{4}$ only etch

Figure 4.15: SEM results with $C F_{4}$

[5],[26]. $7 \mathrm{sccm}$ of sulfur hexafluoride, $\mathrm{SF}_{6}$, was used at a low pressure of $0.2 \mathrm{~Pa}(1.5 \mathrm{mT})$. The ICP at $150 \mathrm{~W}$ was used in addition to the RF bias power of $140 \mathrm{~W}$. The low pressure ICP plasma generated ions with longer mean free path of ions resulting in large ion bombardment making the physical etch predominant [26]. Since $\mathrm{O}_{2}$ attacks and removes photoresist, a recipe with only $\mathrm{CF}_{4}$ at a low pressure of $6 \mathrm{mT}$ was tested.

\begin{tabular}{|c|c|c|c|c|c|}
\hline Gases & Flow Rate(sccm) & Pressure (mT) & RF Power (W) & ICP Power (W) & Time \\
\hline $\mathrm{CF}_{4}$ & 20 & 6 & 140 & 150 & $15 \mathrm{~m}$ \\
\hline
\end{tabular}

The plasma flickered during the process. This must be because the difference in the etchant species resulted in a lower density of ions and free electrons and the plasma could not be sustained at that low pressure. The picture (Fig.4.15(b)) shows the debris left after etching. The compounds of sodium, aluminum and potassium seem to be hard to remove. It seems that the oxides in Borofloat act as 'seeds' from which the non-volatile compounds grow and are thus stubborn to the etch process. However, these should not affect the optical properties of the grating much as they are under hundred nanometers in size. 


\subsection{2 $\mathrm{SF}_{6}$ etch}

\subsubsection{Resist mask}

We then attempted to etch using $\mathrm{SF}_{6}$. However, due to limitations of the Oxford PlasmaLab $80+$ system, stable plasmas at very low pressures and low flow rates could not be attained. The chamber would not pump down to less than $8 \mathrm{mT}$. A reduced flow rate of $8 \mathrm{sccm}$ at $9 \mathrm{mT}$ gave a stable plasma. To compensate the inability of etching at a lower pressure, the RF bias power was increased to $200 \mathrm{~W}$ from $140 \mathrm{~W}$ to increase the ion energy and contribute towards a more physical etch.

\begin{tabular}{|c|c|c|c|c|c|}
\hline Gases & Flow Rate $(\mathrm{sccm})$ & Pressure $(\mathrm{mT})$ & RF Power $(\mathrm{W})$ & ICP Power $(\mathrm{W})$ & Time \\
\hline $\mathrm{SF}_{6}$ & 8 & 9 & 200 & 150 & $4 \mathrm{~m}$ \\
\hline
\end{tabular}

The picture (Fig.4.16(a)) shows a vertical sidewall profile. This is because of the anisotropic etching and improved photolithography. The non-volatile by-products still exist. The combination of the gas and increase in the RF bias power resulted in improving the sidewall and surface roughness as seen compared to the previous recipe.

To replicate the grating structure a mask with $10 \mu \mathrm{m}$ lines with varying duty was used.

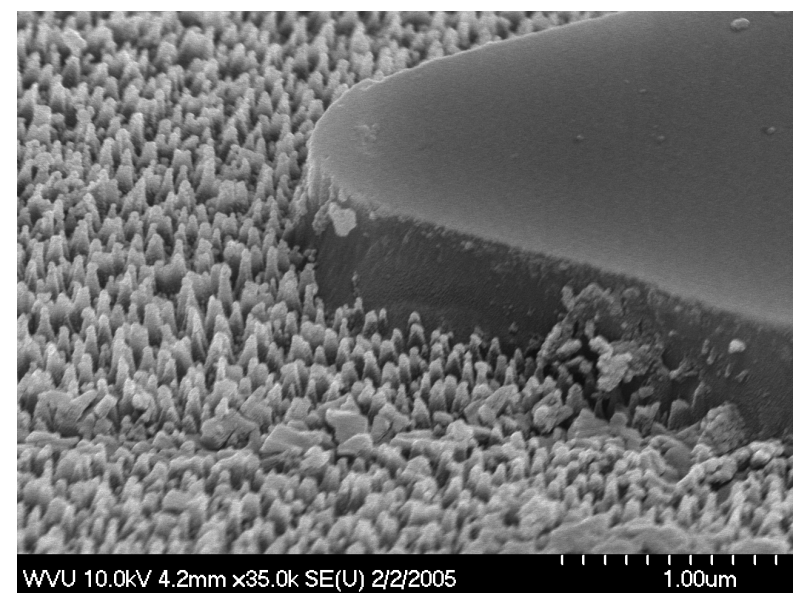

(a) Vertical sidewall

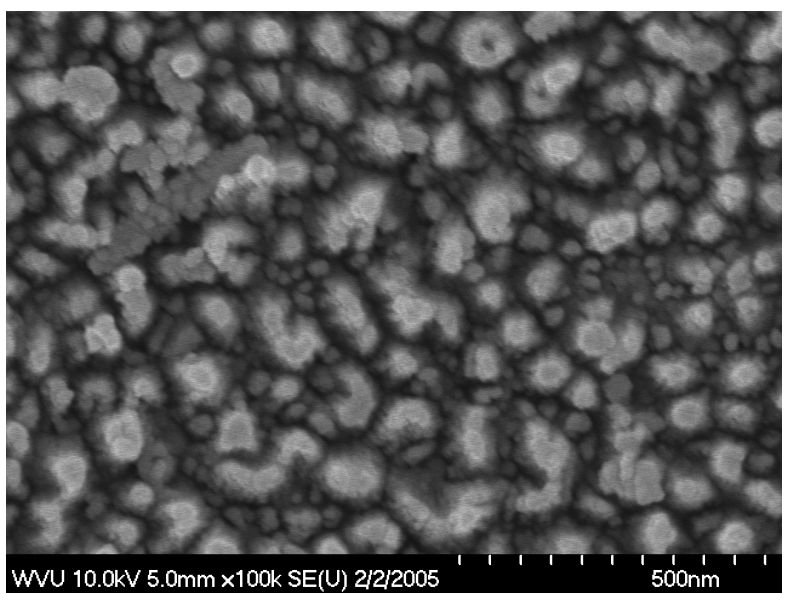

(b) Top view with reduced debris

Figure 4.16: SEM results of $S F_{6}$ etch with photoresist as mask 
The same $\mathrm{SF}_{6}$ recipe was used to etch into borofloat. Interesting results were obtained. When etched for 2 mins (recipe above for 4mins), less resist was removed during PR strip (10 min O2 strip). The SEM images showed the remaining resist (Fig.4.17(a,b)). Since the resist profiles after photolithography were sloped, in a second (longer-4mins) etch (Fig.4.18(a,b)), the sloped sidewalls were transferred to the borofloat. As the resist sidewalls were etched, Borofloat was exposed to $\mathrm{SF}_{6}$ etching resulting in sloped sidewalls.

A loading effect was also seen. In areas with denser lines, the sidewalls were vertical and

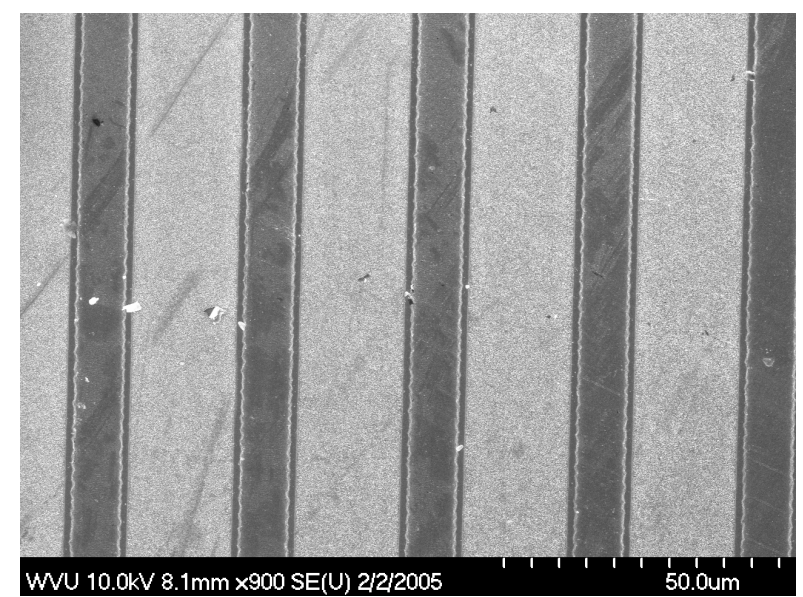

(a) $20 \mu \mathrm{m}$ spacing with resist remaining

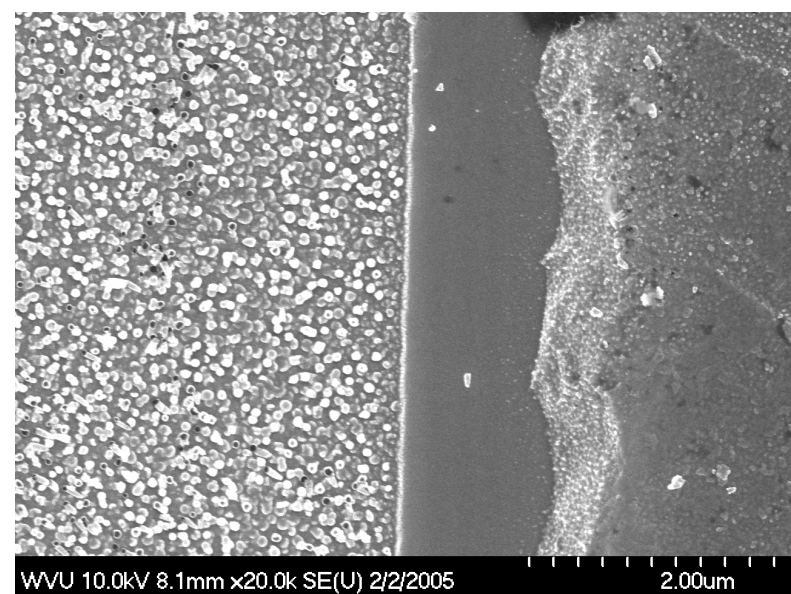

(b) Top view showing resist pull back

Figure 4.17: SEM results of $S F_{6}$ etch (2 mins.) of $10 \mu \mathrm{m}$ lines

the etch rate was higher, as compared to the less dense area (less number of lines, more area etched)(Fig.4.18(b,d)). This demonstrates the loading effect commonly seen on a non-uniform pattern. The etched depth for different periods is show in Fig.4.19.

Since low pressures down to $1 \mathrm{mT}$ could be sustained, the recipe in [26] was tried out. This was possible by "training" the valve to open such that pressure was low.

\begin{tabular}{|c|c|c|c|c|c|}
\hline Gases & Flow Rate $(\mathrm{sccm})$ & Pressure (mT) & RF Power (W) & ICP Power (W) & Time \\
\hline $\mathrm{SF}_{6}$ & 7 & 1 & 140 & 150 & $5 \mathrm{~m}$ \\
\hline
\end{tabular}

The process did not give results as obtained in the paper. The sidewalls were sloped and etched. 


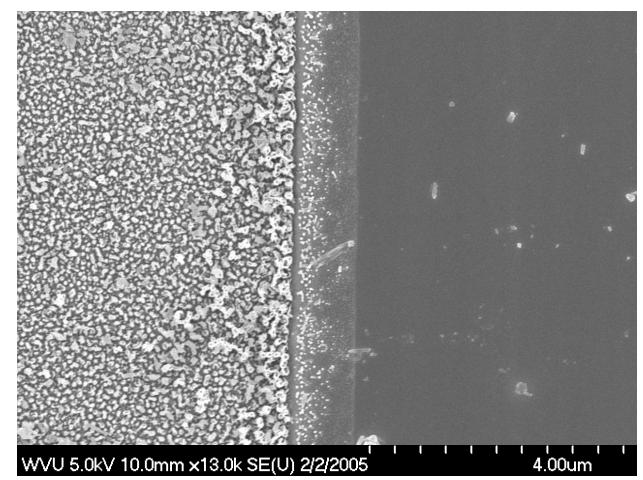

(a) Sloped borofloat sidewall

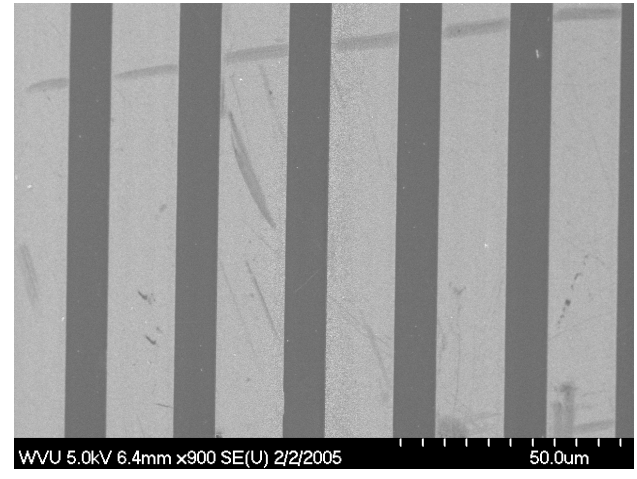

(c) $15 \mu \mathrm{m}$ spacing

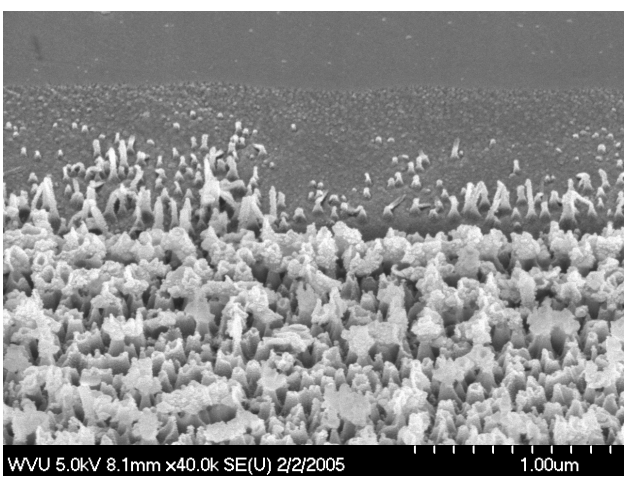

(b) Sidewall being etched

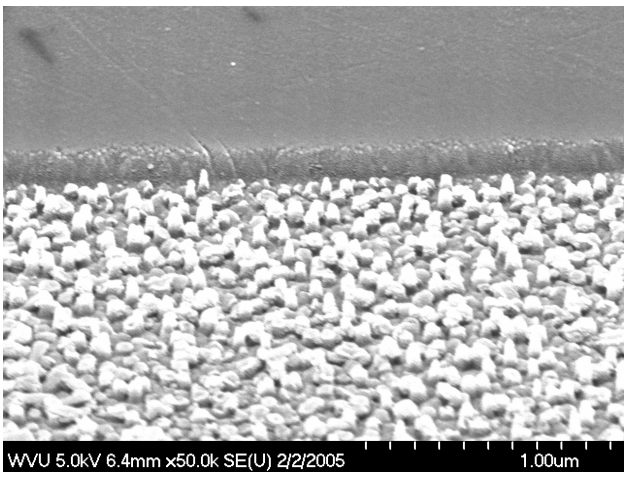

(d) Reduced etch depth

Figure 4.18: SEM results of $S F_{6}$ etch(4 mins.) of $10 \mu \mathrm{m}$ lines

Depth vs. line spacing

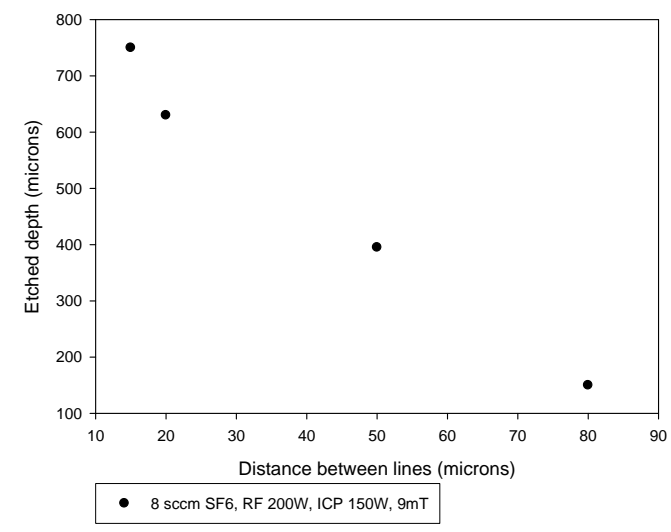

Figure 4.19: Etch depth plot for $10 \mu \mathrm{m}$ lines with varying period 
The sidewalls could be due to bad photolithography. The debris was as much or more Fig.4.20

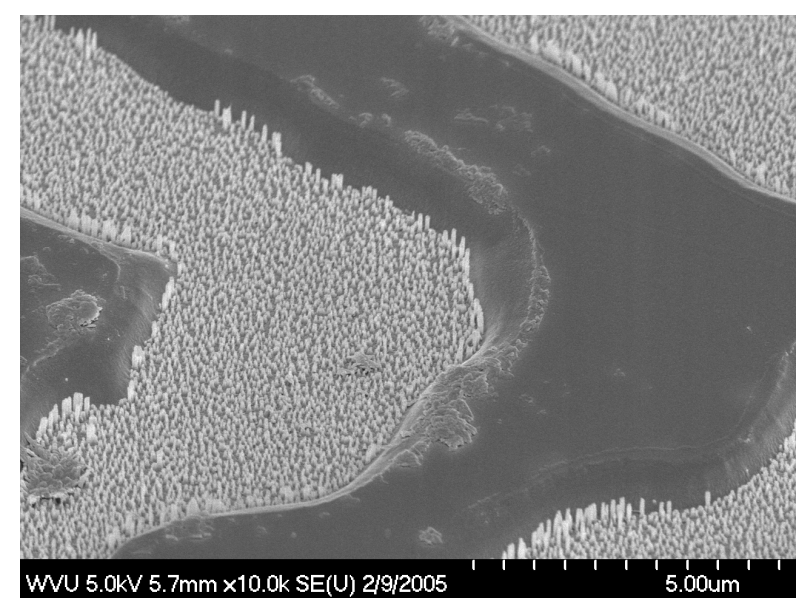

(a) Sloped sidewall

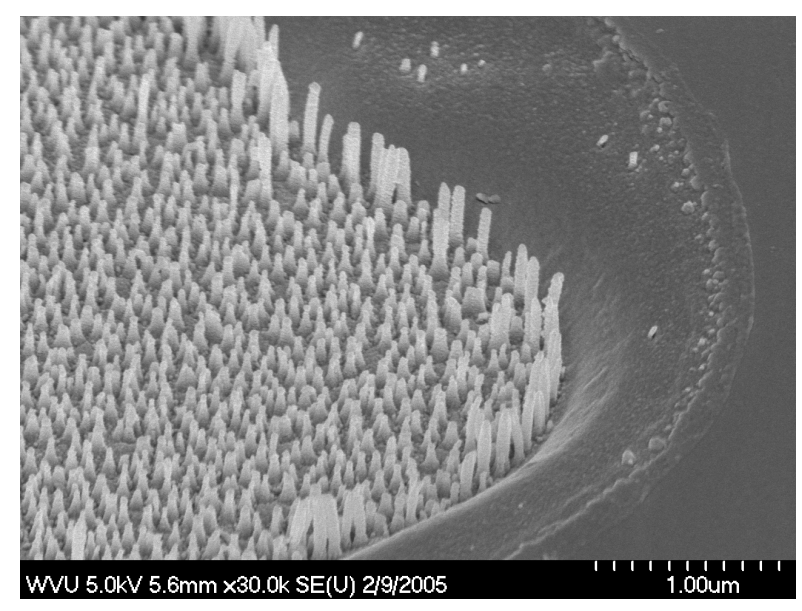

(b) Debris not removed

Figure 4.20: $S E M$ results of low bias power(140W) $S F_{6}$ etch with photoresist as mask

On varying pressure it is found that etch depth increases with reduced pressure Fig.4.21. This confirms the fact that a low pressure increases directionality and energy of ions and hence the etch depth. Also since the removal rate of the volatile products is higher, the surface being etched is exposed to the plasma.

A recipe with higher RF power was tested.

\begin{tabular}{|c|c|c|c|c|c|}
\hline Gases & Flow Rate $(\mathrm{sccm})$ & Pressure $(\mathrm{mT})$ & RF Power $(\mathrm{W})$ & ICP Power $(\mathrm{W})$ & Time \\
\hline $\mathrm{SF}_{6}$ & 8 & 1 & 200 & 150 & $4.5 \mathrm{~m}$ \\
\hline
\end{tabular}

As expected, the process with the higher RF power etched faster (ions with increased energy). The debris was a little less. However, the sidewalls were sloped (this could be because of poor sidewall of photoresist) Fig.4.22. 


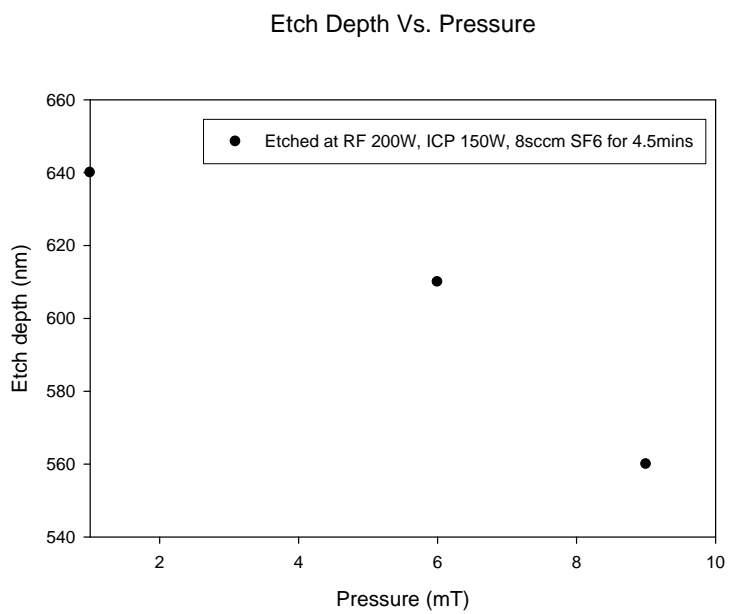

Figure 4.21: Plot of etch depth as pressure is varied

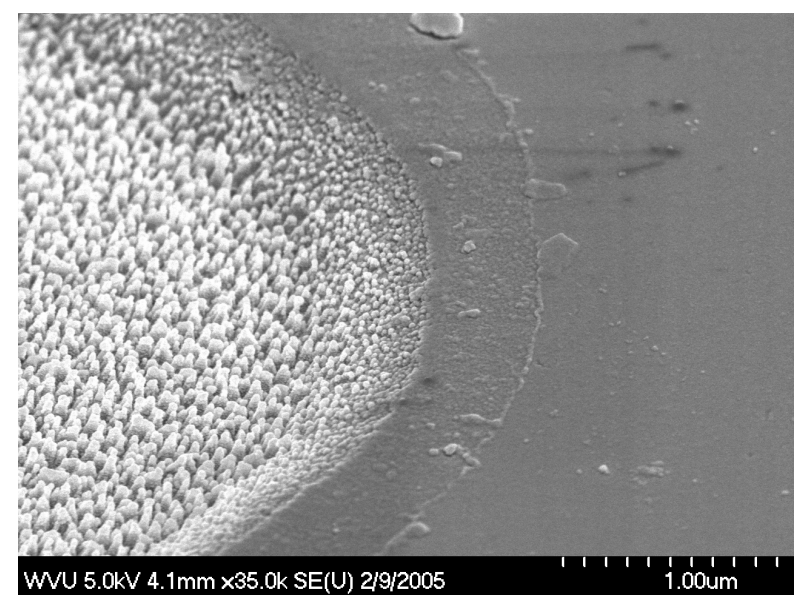

(a) Sloped sidewall

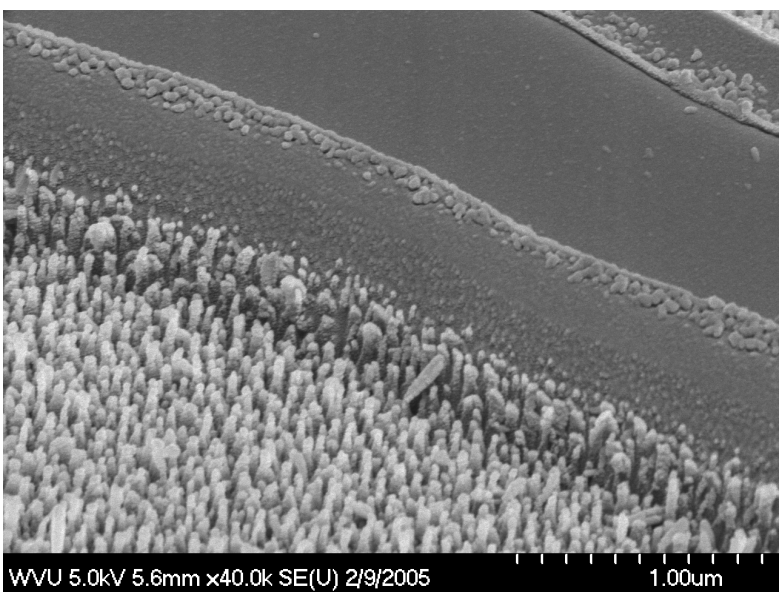

(b) Debris

Figure 4.22: SEM results of high bias power(200W) $S F_{6}$ etch with photoresist as mask

From the above recipes and results, it was found that including ICP power allowed for higher RF bias powers and low pressures. This is further re-iterated in some of the following experiments. Also the etch rate increases with increasing RF bias power.

\subsubsection{Cr mask}

Since the photoresist sidewalls were transferring their slope to the etched borofloat, chrome's ability to improve this and it's use as a mask(etch rate of $\sim 1 \mathrm{~nm} / \mathrm{min}$ [25]) for RIE were tested. 
Initially all the evaporated chrome was lifted off. This was because of resist remaining after exposure/development. The image reversal procedure was followed for the positive process until the first exposure followed by development. However, 15 secs. of exposure (@2.5mW/ $\mathrm{cm}^{2}$ ) is insufficient to fully expose the resist in the positive process (no post bake and flood exposure).The correct exposure time was found to be 55 secs. for complete removal of resist by the positive process.

It was also found that a descum process (given in table below) is needed. Inspite of correct exposure/development times, it is necessary to remove the residual photoresist or HMDS before Chrome deposition. Without it, all the chrome is lifted off and no pattern is left. Since a uniform and isotropic process is desired, higher pressure and flow are used. The ICP is not required in such a process. The oxygen clean is done for a short time as the uniform etch process should not be allowed to remove significant amount of the resist in the feature areas.

\begin{tabular}{|c|c|c|c|c|c|}
\hline Gases & Flow Rate(sccm) & Pressure (mT) & RF Power (W) & ICP Power (W) & Time \\
\hline $\mathrm{O}_{2}$ & 30 & 300 & 150 & 0 & $30 \mathrm{~s}$ \\
\hline
\end{tabular}

To confirm the fact that evaporated chrome was better for lift-off than sputtered chrome, both were tried. Sputtered chrome also lifted-off in acetone $(10 \mathrm{~min}$. soak $+5 \mathrm{~min}$ ultrasound, $5 \mathrm{~min}$ methanol, blow dry $\mathrm{N}_{2}$ ).

Chrome sputtering:

\begin{tabular}{|c|c|c|c|c|c|c|}
\hline $\begin{array}{c}\text { Vacuum } \\
\text { Pressure }\end{array}$ & Gas Flow & Gas Pressure & $\begin{array}{c}\text { Target } \\
\text { Voltage }\end{array}$ & $\begin{array}{c}\text { Deposition } \\
\text { Power }\end{array}$ & $\begin{array}{c}\text { Pre-deposition } \\
\text { Time }\end{array}$ & $\begin{array}{c}\text { Deposition } \\
\text { Time }\end{array}$ \\
\hline $5 \times 10^{-} 6$ & $30.1 \mathrm{sccm}$ & $60.05 \mathrm{mbar}$ & $442 \mathrm{~V}$ & $0.1 \mathrm{KW}$ & $1 \mathrm{~m}$ & $4 \mathrm{~m}$ \\
\hline
\end{tabular}

Chrome of about $160 \mathrm{~nm}$ is sputtered. Precautions need to be taken not to contaminate the surface before sputtering (water vapor or any dirt/dust). Chrome may crack or swell under vacuum. The sputtered/evaporated chrome on lift-off formed a perfect replica of the mask pattern 
(Fig.4.23).

To compare the effects of using a chrome mask with a resist mask, the same process of 8

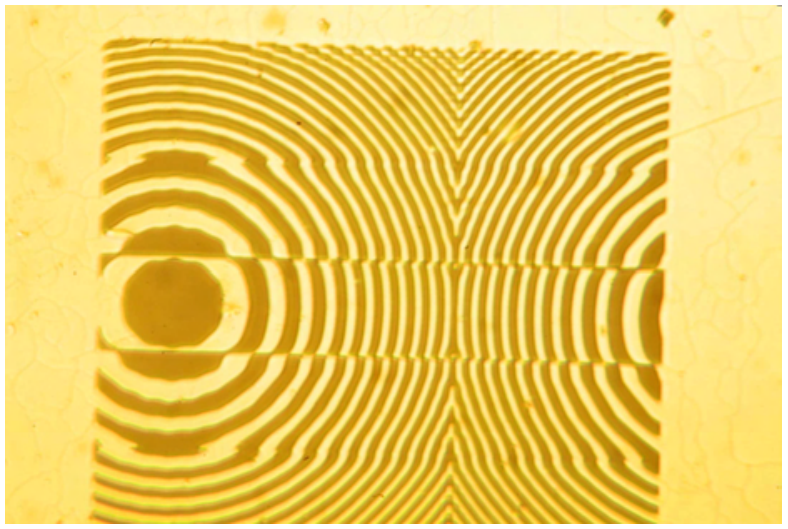

(a) Lens structure

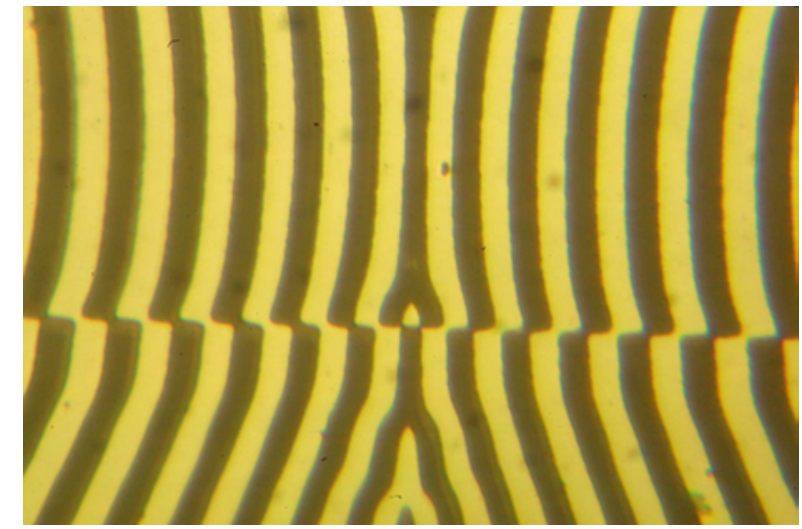

(b) Closer view

Figure 4.23: Pattern in evaporated chrome after lift-off

sccm of $\mathrm{SF}_{6}, 200 \mathrm{~W}$ RF, $150 \mathrm{~W}$ ICP and a pressure of $1 \mathrm{mT}$ was carried out for $4.5 \mathrm{mins}$. After RIE, the chrome is removed by wet etching for 15-20 mins. using chrome etchant (Transene). The sample is then immersed in $\mathrm{H}_{2} \mathrm{SO}_{4}: \mathrm{H}_{2} \mathrm{O} 5: 100$ for 5 mins and then in DI water for 5 mins. This prevents the white residual film (seen after chrome etch) from forming on the borofloat.

With sputtered $\mathrm{Cr}$ as a mask, the etch rate has increased and the sidewalls have improved. However, now instead of sloped sidewalls, an undercut is seen. Also the top surface is cracked along the edge Fig. 4.24. The debris is shorter in length.

Evaporated chrome gave better results Fig.4.25. Instead of sputtering, 200nm of Cr was deposited by e-beam evaporation. The sample was then etched using the same recipe above. The etch rate was about $139 \mathrm{~nm} / \mathrm{min}$ (depth of $600-640 \mathrm{~nm}$ etched in $4.5 \mathrm{mins}$.). Sidewalls were vertical. No undercut and no cracking of surface were seen.

The next recipe implemented was with higher RF power of $250 \mathrm{~W}$. The etch rate was comparable (slightly higher $145 \mathrm{~nm} / \mathrm{min}$ ). The sidewalls are vertical and the debris seems shorter and is different (not pointed cones, but cones cut at the top) Fig.4.26.

Next a recipe with lower ICP of $100 \mathrm{~W}$ was run. The etch rate reduced (close to $97 \mathrm{~nm} / \mathrm{min}$ ). The sidewalls are vertical and the debris is smaller (cones cut off at base)Fig.4.27. 2 layers 


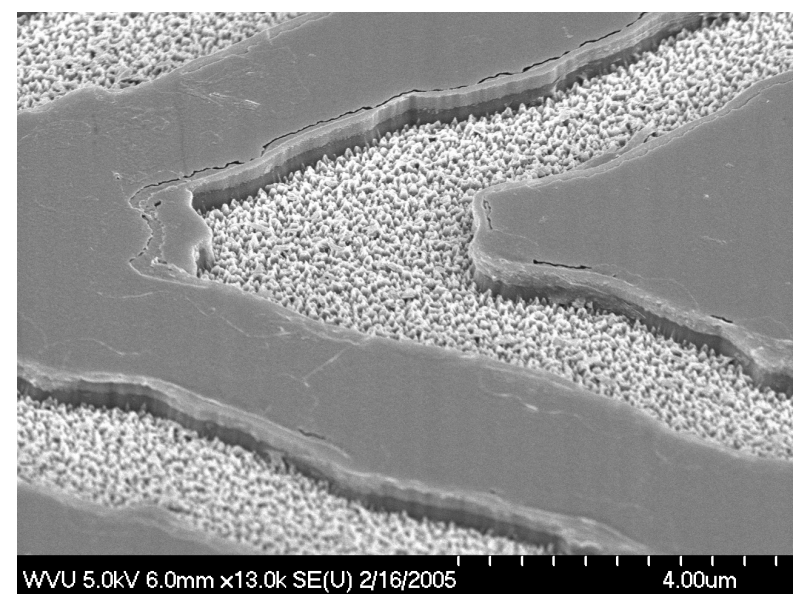

(a) Undercut sidewall

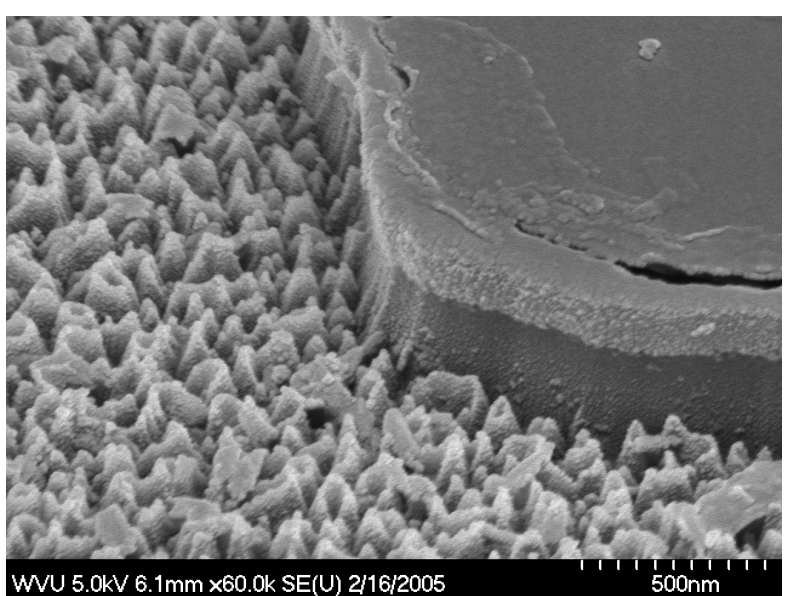

(b) Cracking of chrome

Figure 4.24: Etch with sputtered chrome as mask

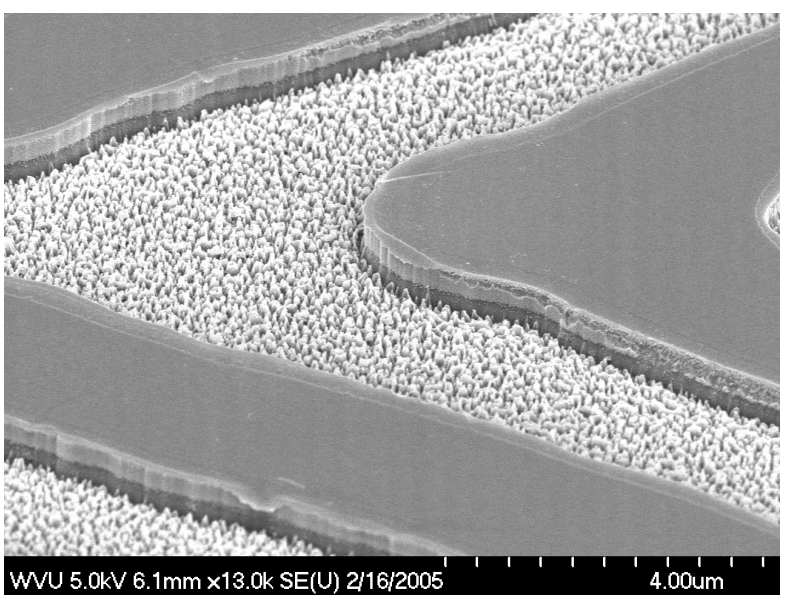

(a) Vertical sidewalls

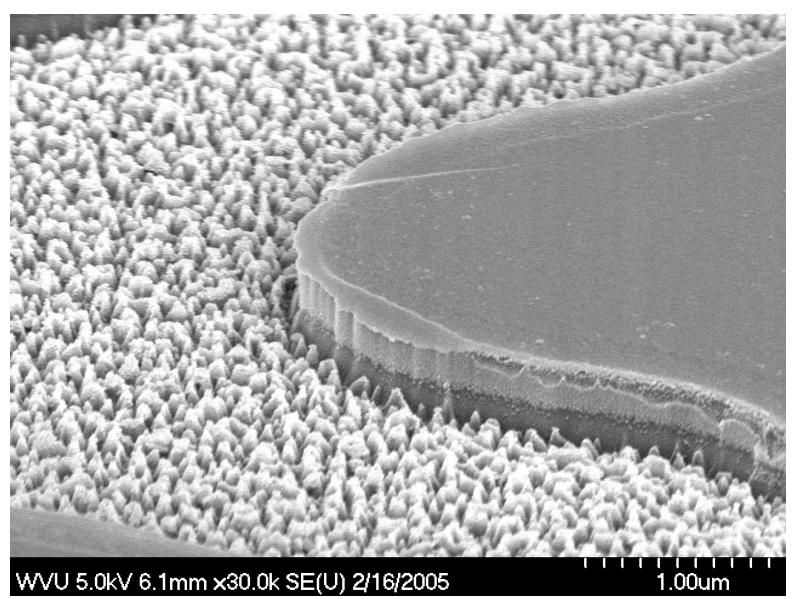

(b) No undercut/cracking

Figure 4.25: Etch with evaporated chrome as mask

have been distinctly visible in the above pictures (except for low ICP) for large etch depths. This may be due to the polishing process done on both sides of Borofloat as the last step in the manufacturing process.

Both the high RF and low ICP have reduced the debris and using both at the same time should smoothen the surface. 


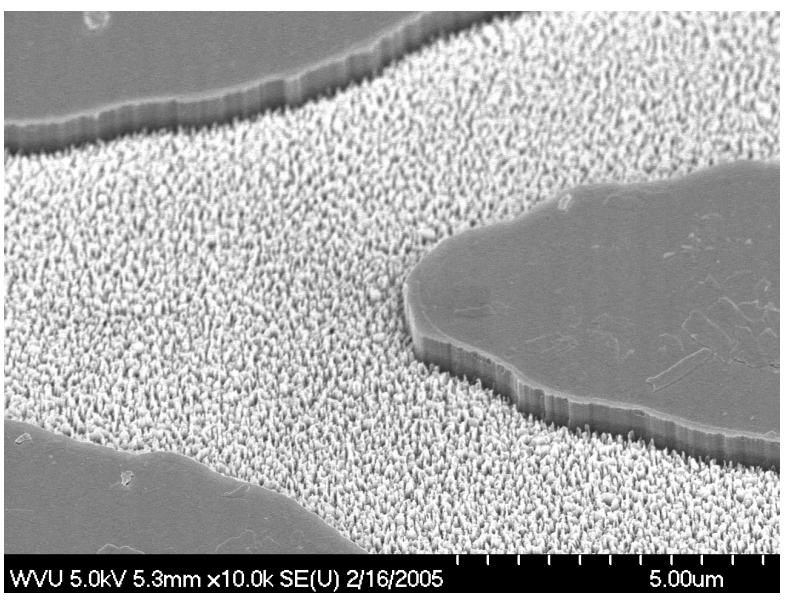

(a) Vertical sidewalls

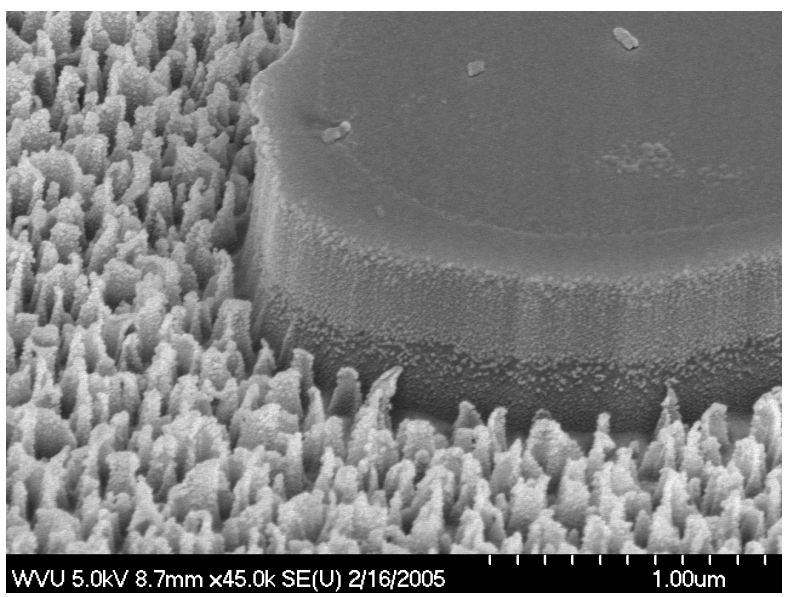

(b) Shorter debris

Figure 4.26: Etch at RF 250W

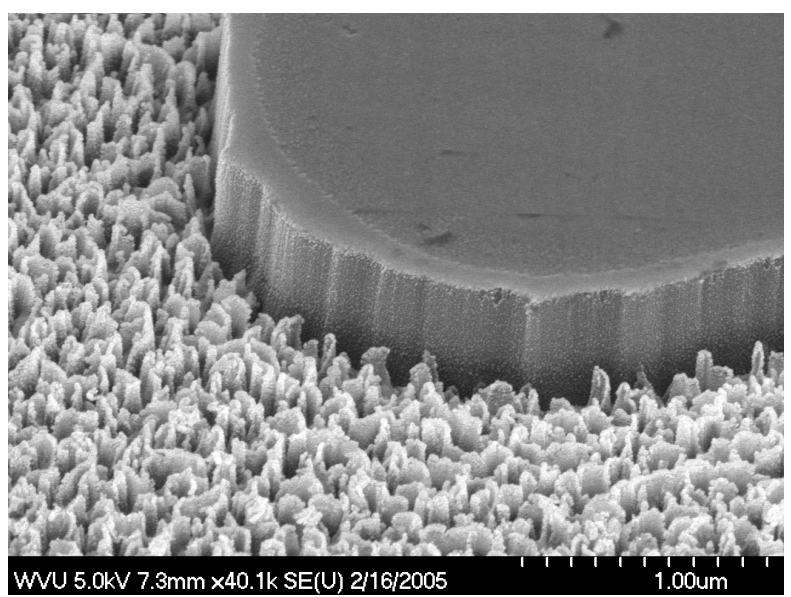

(a) Vertical sidewalls

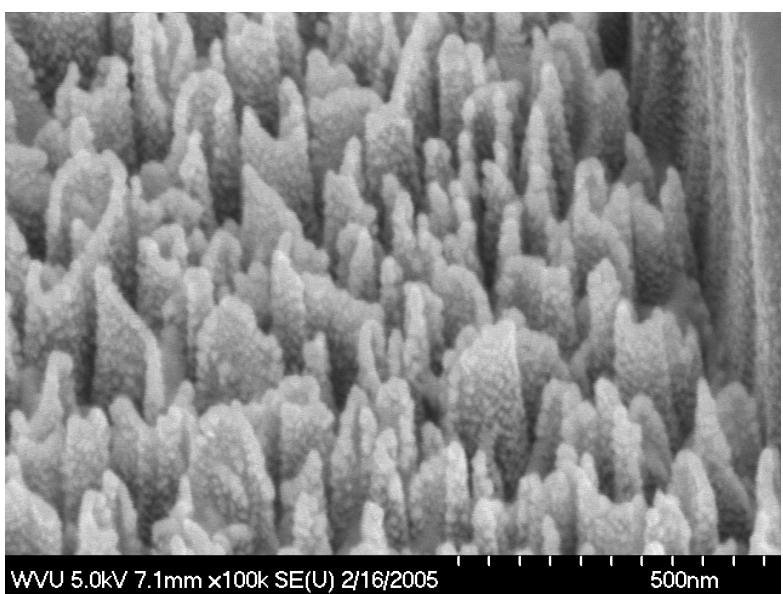

(b) Smaller debris

Figure 4.27: Etch at ICP 100W

\subsubsection{Varying RF and ICP}

To understand the effects of the RF bias power and ICP power, various experiments were carried out. All processes were carried out at $1 \mathrm{mT}$ for $4.5 \mathrm{mins}$ with a $\mathrm{SF}_{6}$ gas flow of $8 \mathrm{sccm}$. The first modified recipe had high RF power (250W) and ICP of 100W. Etch depth was higher ( 600nm on Alpha-step). Also debris was shorter Fig.4.28(a). The second recipe had both high RF (250W) and low ICP (50W). Etch depth was almost the same (420nm). This is because the 
effect of high RF is compensated by the lower density of ions (ICP). The debris is reduced and much smaller! Fig.4.28(b).

The third recipe had RF at 200W and ICP at 50W. As expected the etch rate is lower than

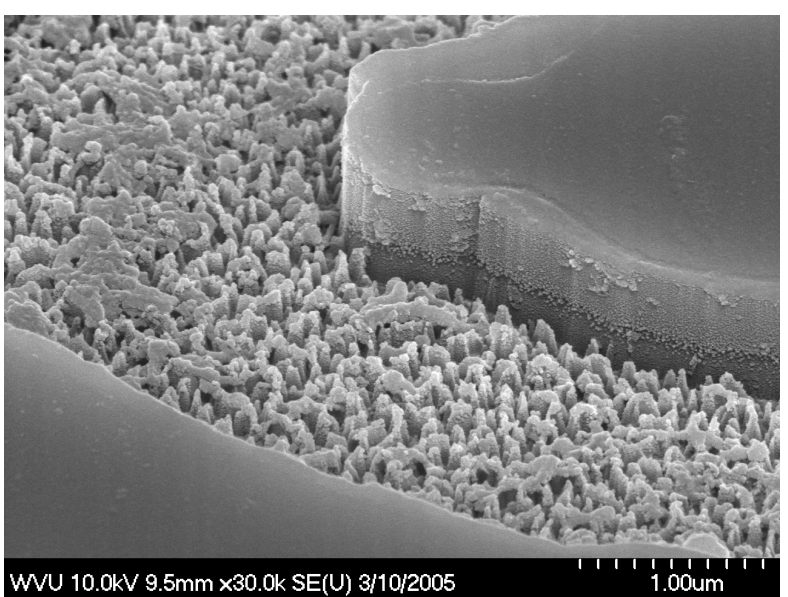

(a) ICP $100 \mathrm{~W}$

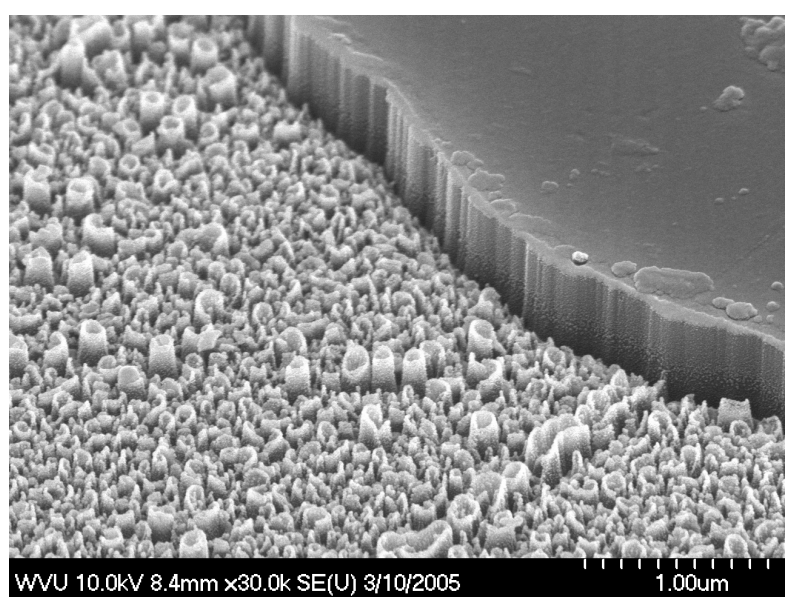

(b) ICP 50W

Figure 4.28: RF250W

above (depth of about 350nm). Surprisingly, the debris is reduced significantly (Fig.4.29(a)). The recipe was repeated again for longer etch time to ensure that results were independent of depth etched (Fig.4.29(b)). The surface roughness is lesser than that obtained in [39] in silica.

In the above recipes, the pressure did not always go down as low as $1 \mathrm{mT}$. The $\mathrm{CM}$ gauge at some times read $8-9 \mathrm{mT}$.

It can be concluded from the above experiments that etch rate depends on both the RF power and ICP power. With an increase in the RF power the energy of the ions is increased resulting in higher etch rates Fig.4.30. An increase in ICP increases the density of ions (without increasing their energy) and also results in high etch rates Fig.4.31.

As a lower ICP gives a controlled etch and has also shown better SEM results, a fourth recipe with no ICP at 200W RF was tried. However, due to the low gas flow and pressure the plasma would not strike. There probably were not enough ions in the charged cloud. This is in agreement with the fact that ICP allows the use of low gas flows and pressures by increasing ion density without increasing the energy of the system significantly. 


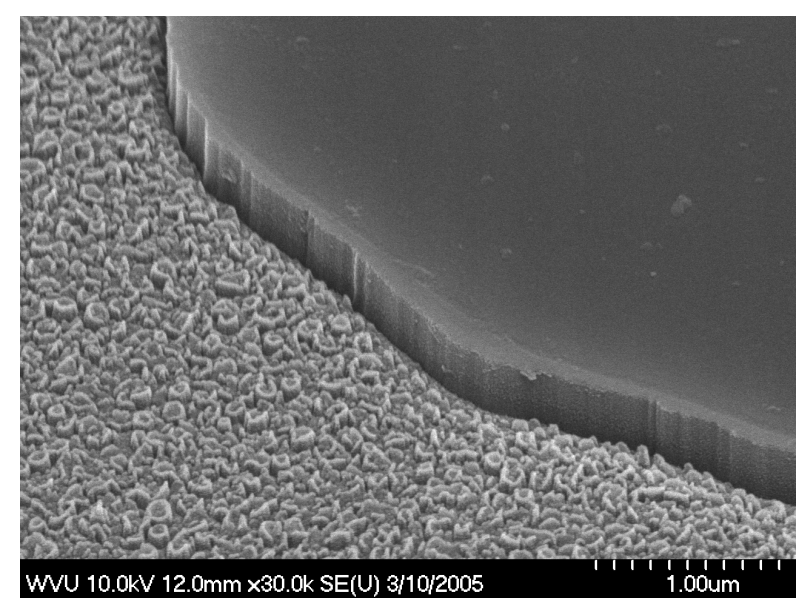

(a) Reduced debris

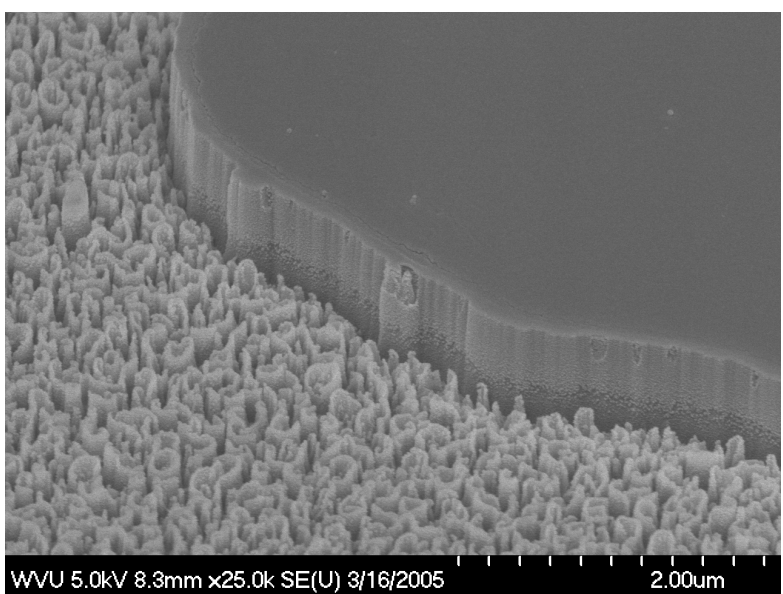

(b) Longer etch

Figure 4.29: Etch at RF200W ICP 50W

Etch Rate Vs. RF Power

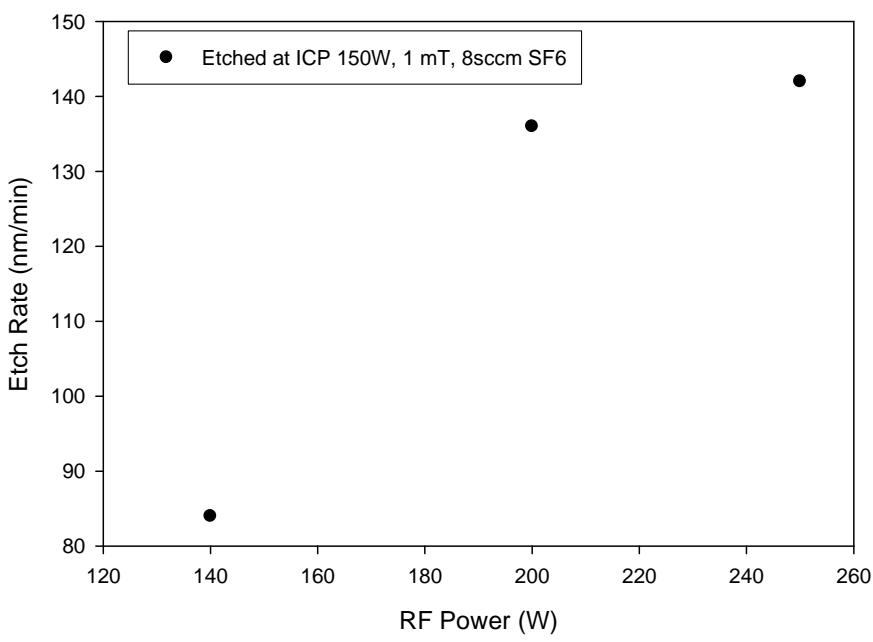

Figure 4.30: Plot of etch rate as RF power varies

Without the ICP, an $\mathrm{SF}_{6}$ etch recipe can be run only at $30 \mathrm{sccm}$ of gas flow and at $25 \mathrm{mT}$ (200W RF). Also the etch rate is very slow $(<35 \mathrm{~nm} / \mathrm{min})$. The etched sidewalls scoop in and the surface has a lot of tall debris. 


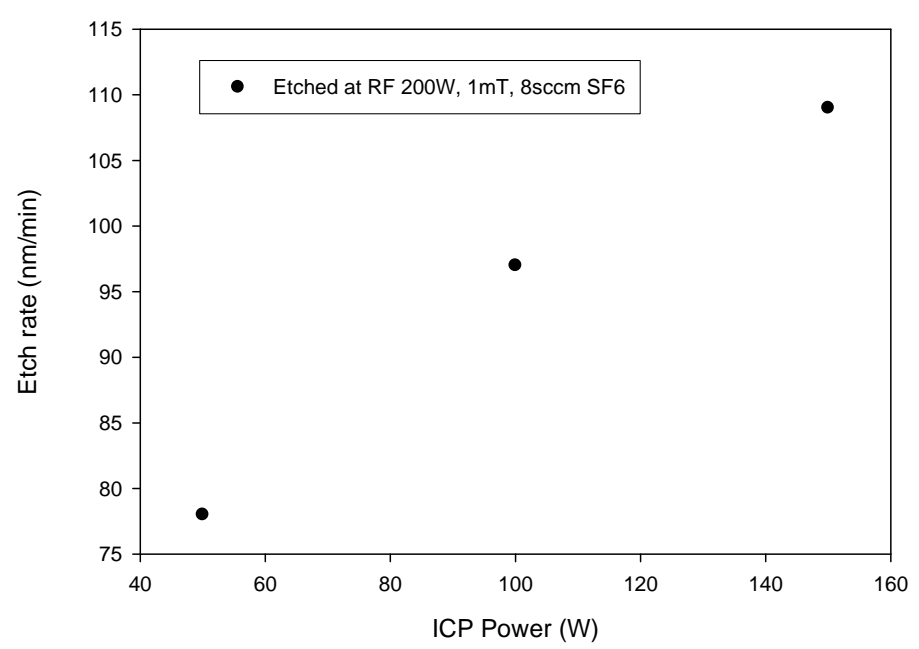

Figure 4.31: Plot of etch rate as ICP power varies

\subsubsection{Post-RIE treatment}

From literature it was found that often post-RIE treatments were done to improve the etched/damaged surface. Acid cleans are commonly used. Since we suspect the metal fluorides of masking areas and forming grass, an acid clean to remove metal ions was tried $\left(\mathrm{H}_{2} \mathrm{O}: \mathrm{H}_{2} \mathrm{O}_{2}\right.$ : $H C l:: 6: 1: 1)$. A $5 \mathrm{sec}$ HF dip followed to remove the underlying oxide debris. This seemed to sharpen the debris features. independent of depth etched (Fig.4.32). HF etch is isotropic and sidewalls begin to scoop in.

To smoothen the etch results the use of a $\mathrm{CF}_{4}$ plasma was then explored post-SF 6 and postacid treatments.

\begin{tabular}{|c|c|c|c|c|}
\hline Gases & Flow Rate(sccm) & Pressure (mT) & RF Power (W) & ICP Power (W) \\
\hline $\mathrm{CF}_{4}$ & 25 & 30 & 150 & 100 \\
\hline
\end{tabular}

$\mathrm{SF}_{6}$-etched borofloat samples were etched with the $\mathrm{CF}_{4}$ plasma after ionic/oxide etch $(\mathrm{HCl}$ : $\mathrm{H}_{2} \mathrm{O}_{2} / \mathrm{HF}$ ). Results of the $\mathrm{CF}_{4}$ plasma on samples with and without HF dip are shown in Fig.4.33. The sharp debris (seen in Fig.4.32) is smoothened out. However it was seen later 


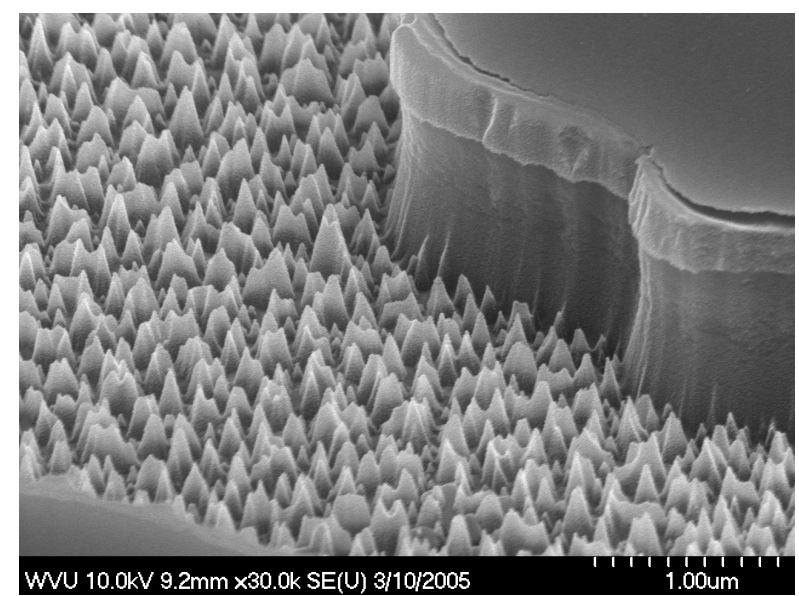

Figure 4.32: Post-RIE ionic clean and HF dip

that when borofloat was $\mathrm{SF}_{6}$ etched at $200 \mathrm{~W} \mathrm{RF}$ and $50 \mathrm{~W}$ ICP and then $\mathrm{CF}_{4}$ etched, the $\mathrm{CF}_{4}$ etch being isotropic ate into the walls.

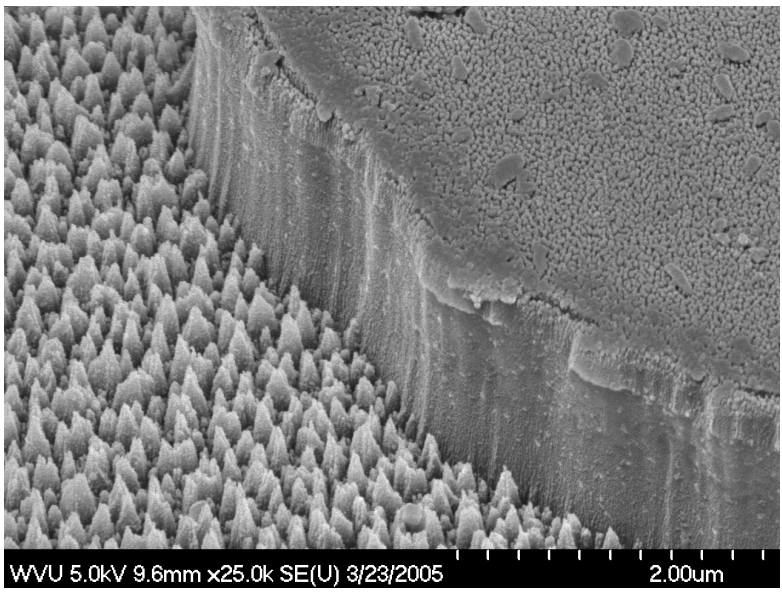

(a) With HF dip

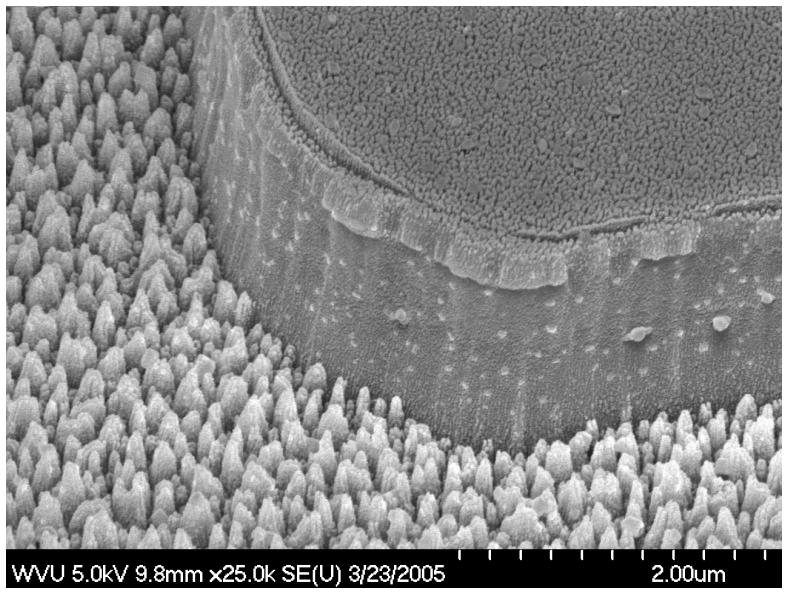

(b) Without HF dip

Figure 4.33: SF6 etched, acid cleaned and CF4 etched

\subsubsection{Silica and Alumina etch}

To investigate how a pure silica surface would etch, we patterned by photolithography a thermal oxide $\mathrm{Si}$ wafer and etched it with $\mathrm{SF}_{6}$. It was etched for 2 mins. as $\mathrm{SiO}_{2}$ was about 
$400 \mathrm{~nm}$ thick and a 4 min. etch removed almost that much Borofloat. The etched $\mathrm{SiO}_{2}$ showed a smooth surface along with vertical sidewalls Fig.4.34.

EDAX pictures (Fig.4.35) of the etched $\mathrm{SiO}_{2}$ and borofloat show the presence of metal

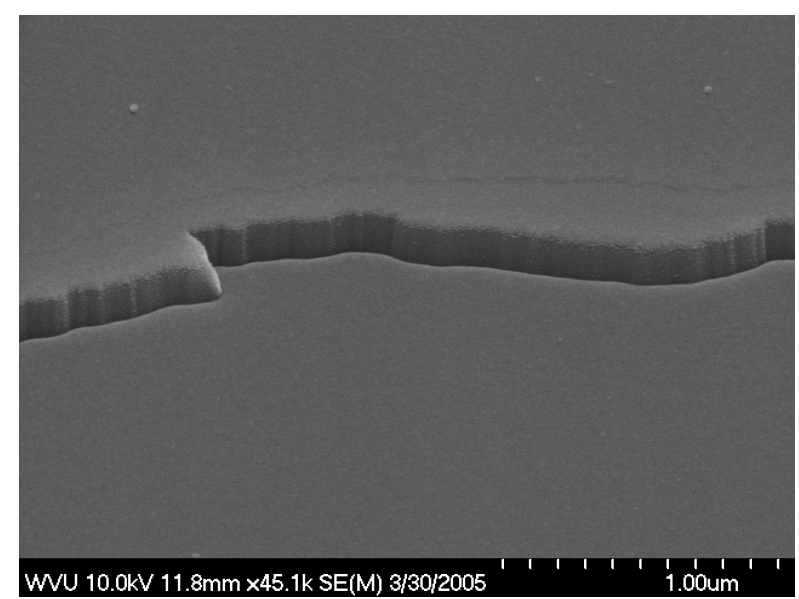

Figure 4.34: Etched silica with smooth surface

oxides in borofloat. The Pt seen in both plots is the metal deposited on the samples to view them in the SEM and is not one of the metal oxides in Borofloat.

C.1EDSUSPRKKolin Brown!ctched Sio2.spe

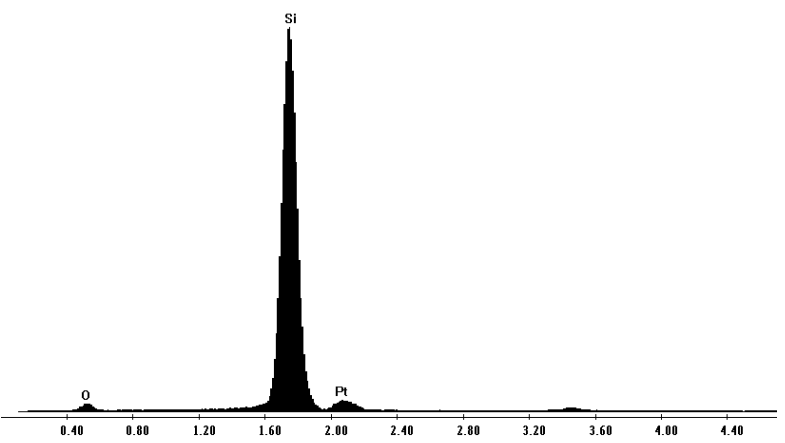

(a) Silica
C:EDSYUSPYKolin Brown!Borofloatspc

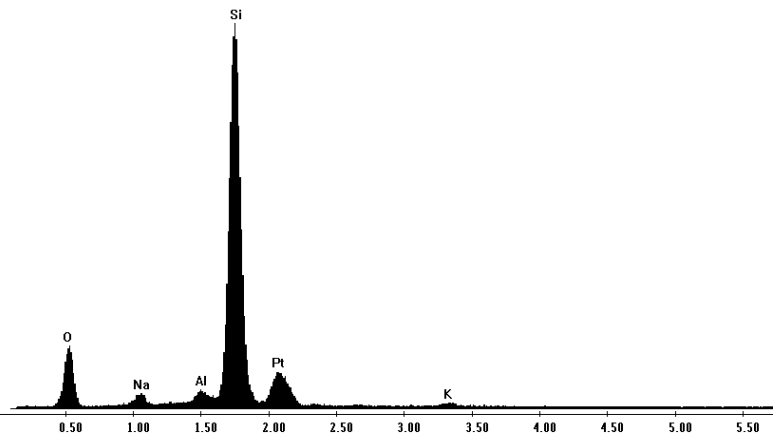

(b) Borofloat

Figure 4.35: EDAX of etched silica and borofloat 
$\mathrm{SF}_{6}$ etches alumina. A sample with $150 \mathrm{~nm}$ of alumina was etched for $1 \mathrm{~min}$. to a depth of $80 \mathrm{~nm}$. A slight surface roughness is seen. The different etch rates of the metal oxides cause the non-uniformity of the etched Borofloat surface.

The ability to etch silica and alumina with a metal mask are useful. Grating structures can

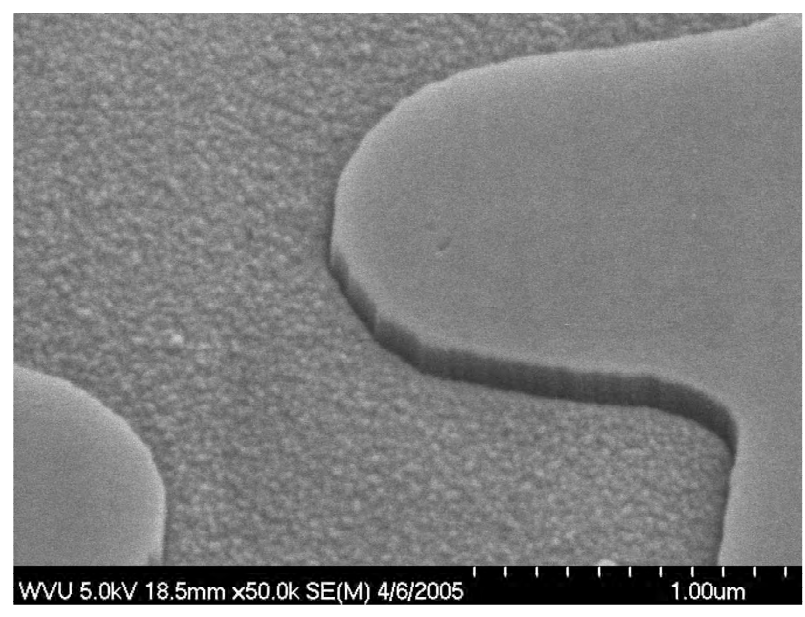

Figure 4.36: Etched alumina

be created in either layer of the bio-sensor with e-beam exposure of resist and then RIE.

\subsection{Direct write and RIE}

Platinum was sputtered on patterned 0.5 $\mu \mathrm{m}$ PMMA/copoly lines (section 4.1.3.4). The bilayer was then lifted off to leave patterned platinum. Platinum was used as the mask to etch silicon. The etched silicon lines have good sidewalls and a reasonably smooth surface.

To etch silicon the following recipe was used.

\begin{tabular}{|c|c|c|c|c|c|}
\hline Gases & Flow Rate(sccm) & Pressure (mT) & RF Power (W) & ICP Power (W) & Time (min) \\
\hline $\mathrm{SF}_{6}$ & 10 & 8 & 220 & 150 & 1 \\
\hline
\end{tabular}

500nm lines were etched in silicon. Silicon has a high etch rate and almost a micron was etched in a minute.

E-beam exposures need to be optimized for a resist bilayer on borofloat. After the grating 


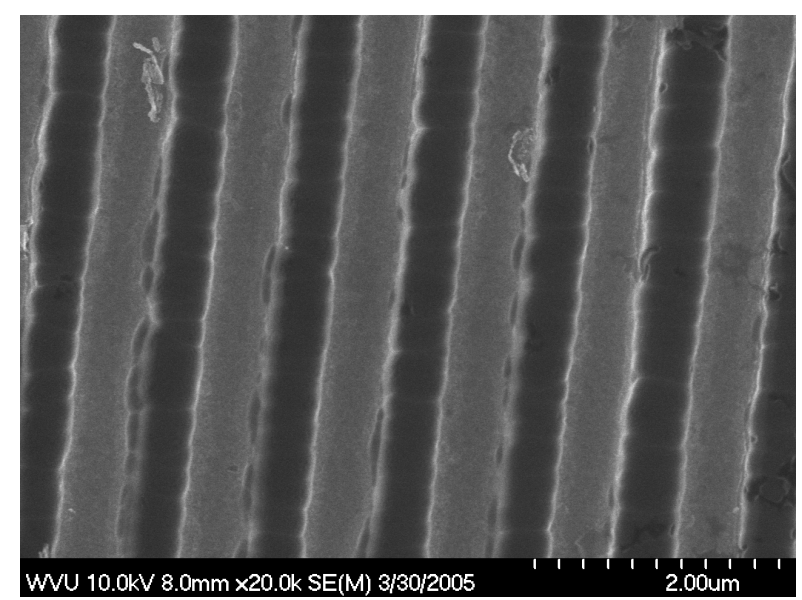

(a) $500 \mathrm{~nm}$ line and space

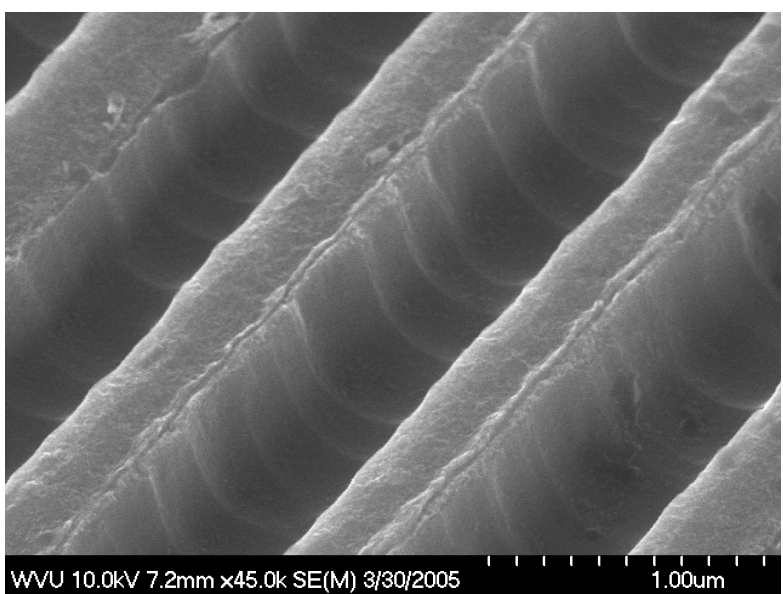

(b) $800-900 \mathrm{~nm}$ deep

Figure 4.37: Si etched with Pt as mask

structure is obtained in resist, metal deposition, lift-off and RIE of borofloat can be done as in the previous sections.

\subsection{Laser Ablation}

The green laser power was measured to be $300 \mathrm{~mW}$. Different size apertures/masks can be used to reduce the beam size and the power. A $1 \mathrm{~mm}$ square mask was used to ablate borosilicate glass. Borofloat, belonging to the same family of glasses, should have the same ablation characteristics.

As the laser beam can be either used in focus to ablate the glass or out of focus, the first step is to determine the appropriate objective distance of the substrate from the focusing lens. When in focus the beam has the smallest size and highest fluence. When defocused the beam spot will be larger and the fluence less. The appropriate objective distance is found by successively ablating holes. The objective distance that gives the hole that is the cleanest and most round is used for subsequent micromachining.

Lines were ablated with different lens positions so that the etch depth could be easily measured. 


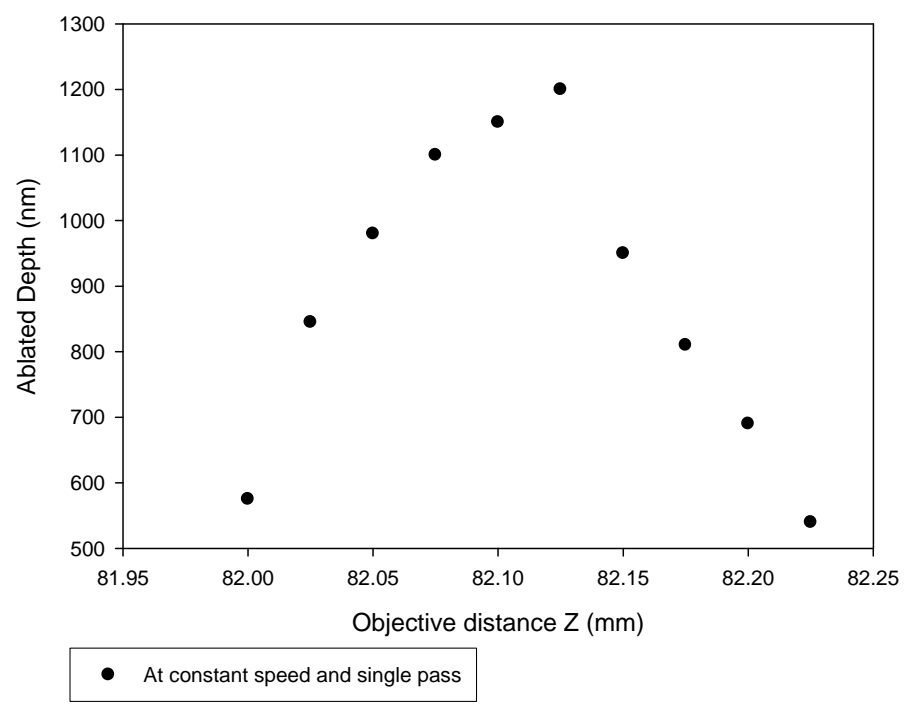

Figure 4.38: Ablated depth as focus lens position is varied

The depth of ablation was measured by ablating lines. For a fixed focus, the depth depends on the speed of the stage and the no. of times (passes) the beam ablates the same line.

For a single pass of the beam, lines were ablated at varying speed. The beam in CVL is stationary and the stage is moved. The ablated depth is compared to the stage speed. The slower the stage moves, the longer the beam is incident on a single spot and hence deeper the line ablated. The discontinuities in the plot Fig.4.39 can be attributed to the inertia of the stage speed and other mechanical factors.

For a fixed speed, the no. of passes of the beam was increased. The more the no. of passes, the deeper the line Fig.4.40.

Thus depth can be controlled by changing either the stage speed or the no. of passes at a fixed position of the focusing lens. Depth can also be varied by changing the lens position.

To create a grating, lines need to be closely spaced. The distance between the lines was 


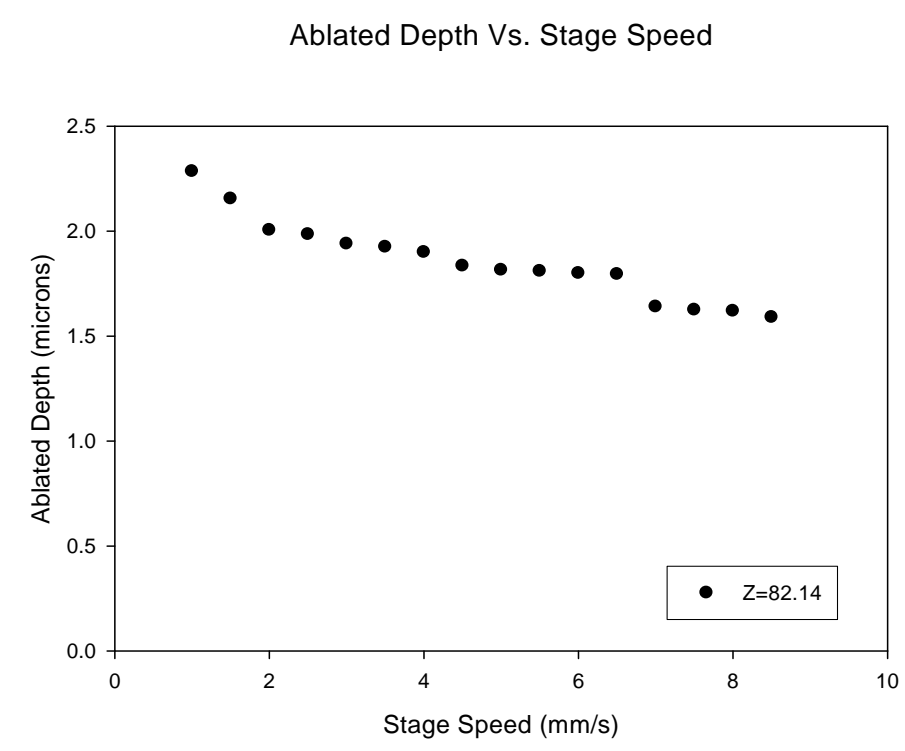

Figure 4.39: Ablated depth with increase in stage speed

Ablated Depth vs. No. of passes

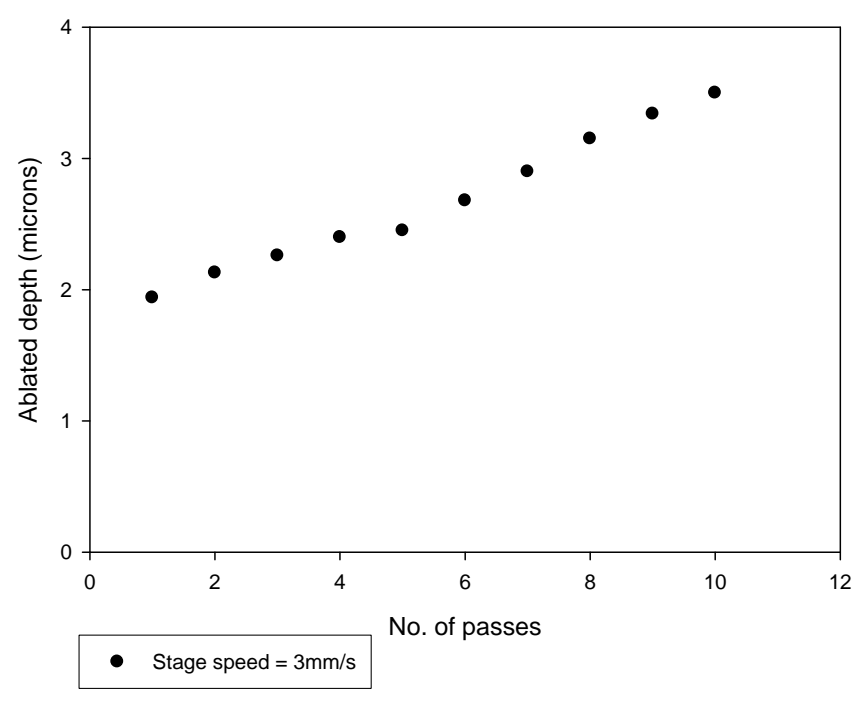

Figure 4.40: Ablated depth for different no. of passes

reduced and it was observed that lines with $10 \mu \mathrm{m}$ spacing were not well formed, but were fused together due to beam overlap. The line width was $10 \mu \mathrm{m}$. Larger line spacings could be ablated. 
Closest line spacings attainable

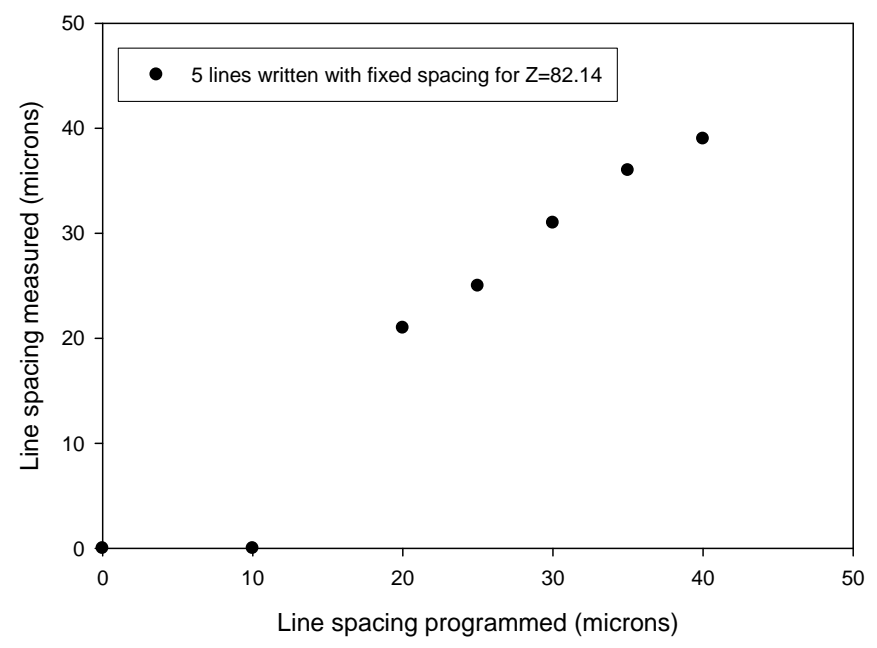

Figure 4.41: Measured vs. desired line spacing

Using a smaller mask may reduce the line width. But creating sub-micron lines by ablation seems close to impossible. Other methods like use of a phasemask need to be explored. The phasemask method could not be implemented due to CVL system issues and is a method that needs to be investigated to create gratings in resist and borofloat. 


\section{Chapter 5}

\section{Optical Testing and Characterization}

\subsection{Diffraction}

Gratings created in PMMA/copolymer(4.1.3.4) were coated with a thin film of Pt. They diffract white light as shown in Fig.5.11.
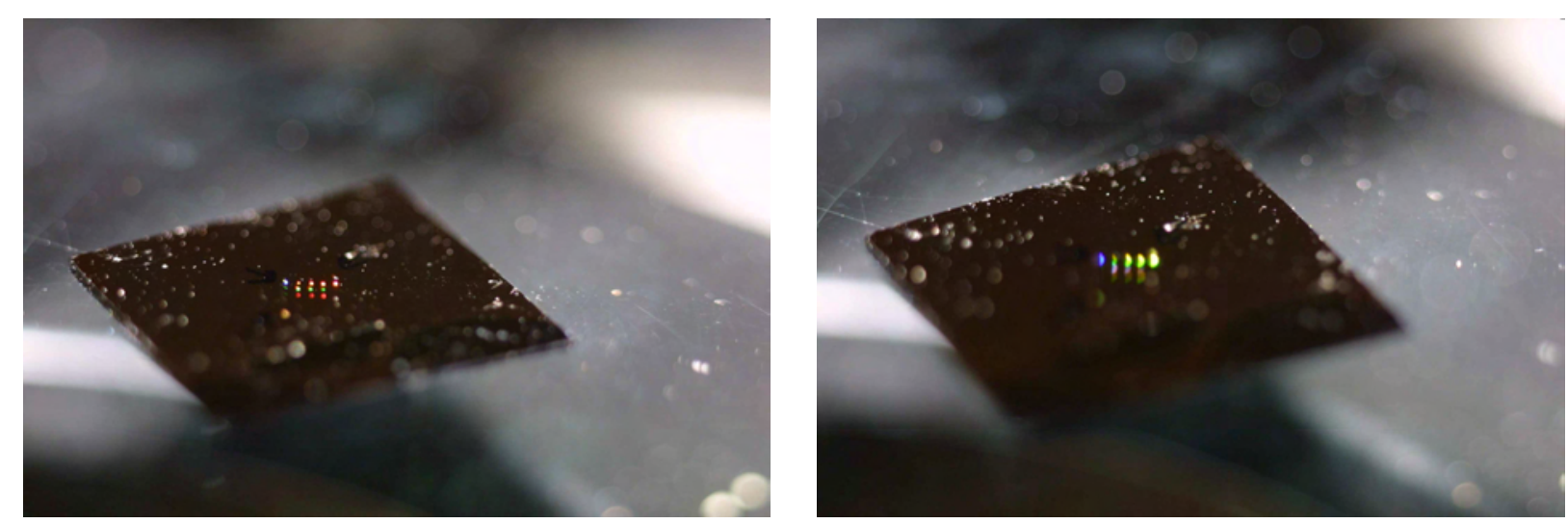

Figure 5.1: Gratings diffracting white light

\subsection{Etched surface testing}

In the previous chapter, the RIE results on borofloat showed some debris. To demonstrate that they do not cause any speckling, the following optical set-up was used Fig.5.2. HeNe light was expanded by a Keplerian lens system. The expanded beam was incident on a piece of optically flat Borofloat at a grazing angle. Interference fringes were formed. The image was captured by 


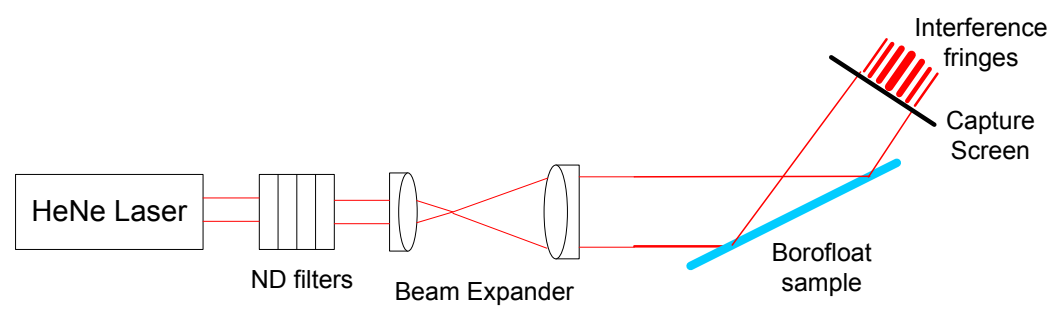

Figure 5.2: Optical set-up to obtain inteference fringes

a digital camera using white paper as a screen. Neutral density (ND) filters were used to reduce the beam intensity.

The etched Borofloat was then placed in the path of the HeNe light and a similar image was obtained. No speckling was seen visually Fig.5.3(a),(b). Hence the etched surface does not significantly alter the optical properties of the incident HeNe light.

This is further demonstrated by comparing the intensity plots Fig5.3(c). The etched Borofloat causes a slight reduction in intensity. However, the etched Borofloat image is smaller (less no. of fringes) than the unetched one. Hence, the two don't match in intensity. This could be either due to absorption in the etched borofloat or more likely due to the inability to take identical pictures with the same set-up for both the Borofloat samples.

By zooming in on the same part of the digital images, the plot shows no speckling Fig5.3(d).

Another method to study the surface roughness effects is to transmit light through an etched sample to be incident on an unetched sample (as above) and compare with normal transmission Fig.5.4.

The intensity plots in both cases overlap Fig5.5. No significant difference is seen. The surface roughness is thus not a major factor and should be acceptable for all optical applications. 


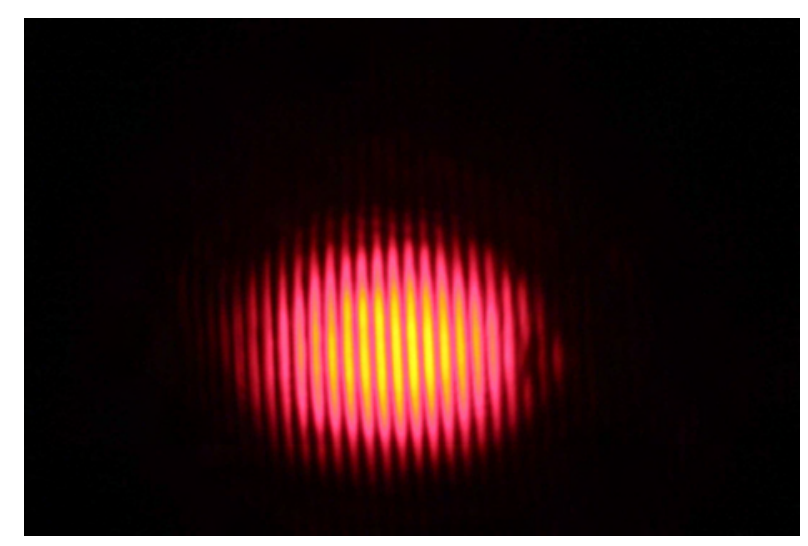

(a) Optically flat

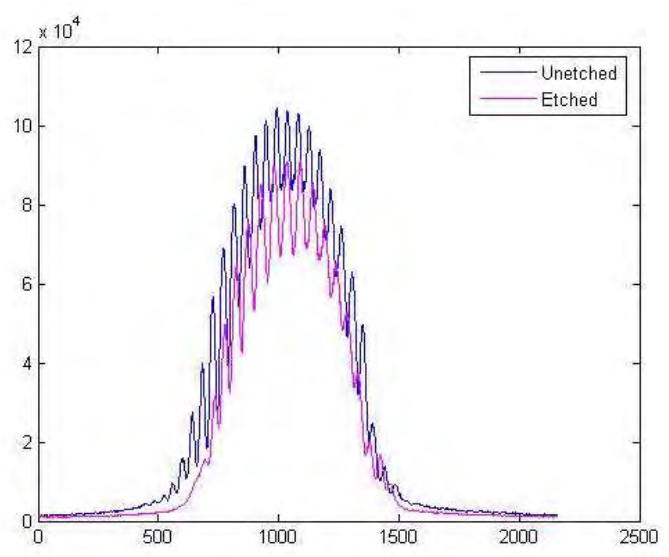

(c) Intensity plot

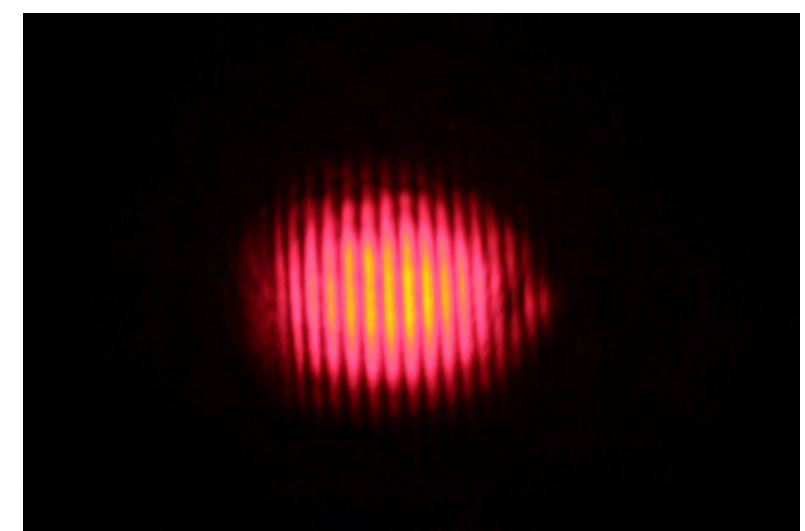

(b) Etched

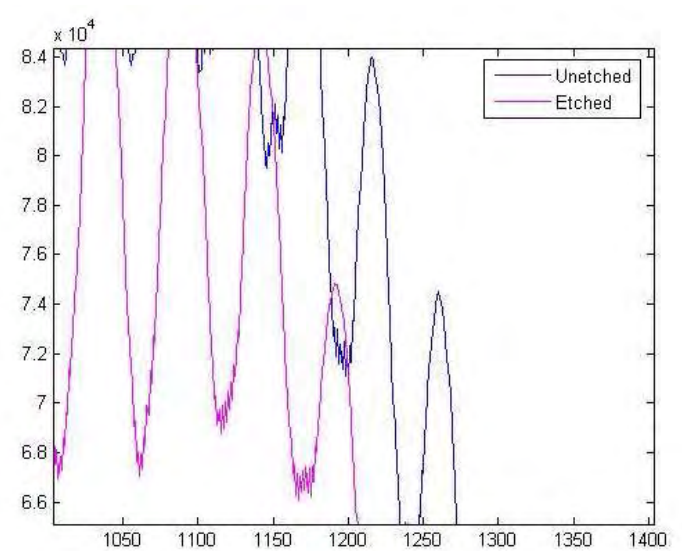

(d) Zoomed in

Figure 5.3: Intensity comparison of Interference fringes of optically flat and etched Borofloat

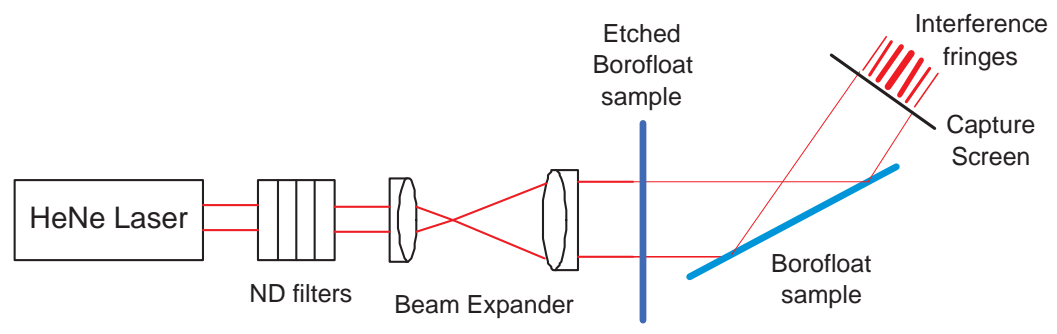

Figure 5.4: HeNe light transmitted through etched Borofloat 


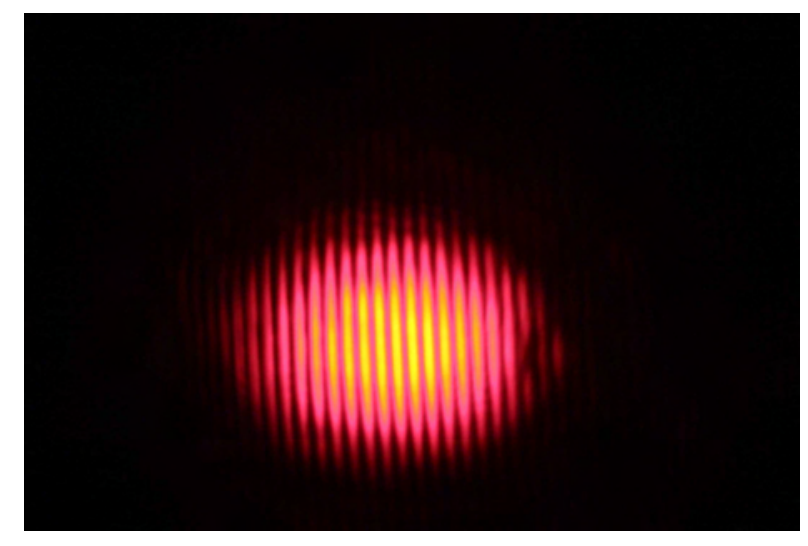

(a) Air

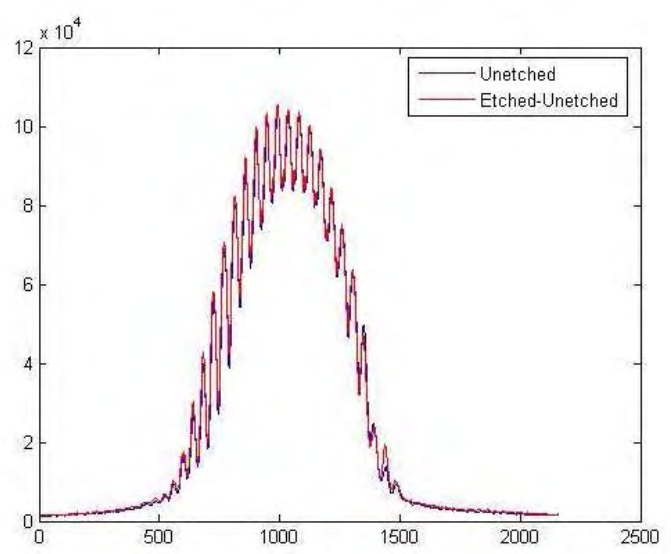

(c) Intensity plot

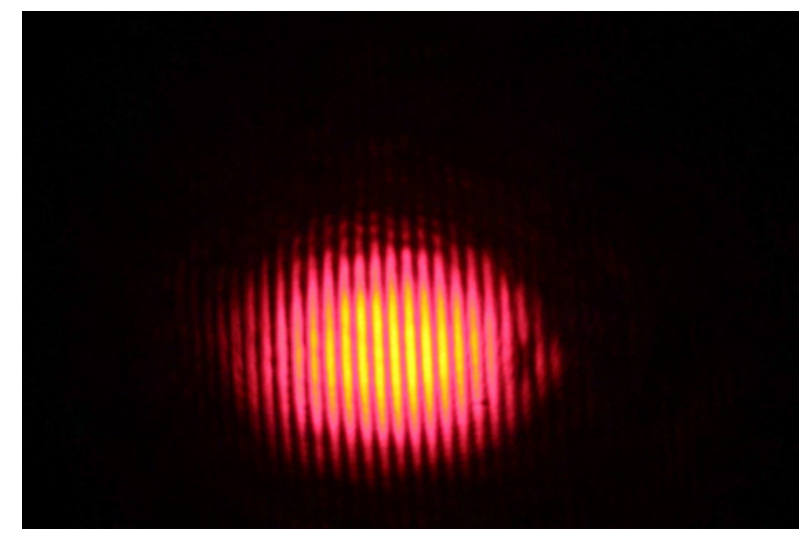

(b) Through the Etched sample

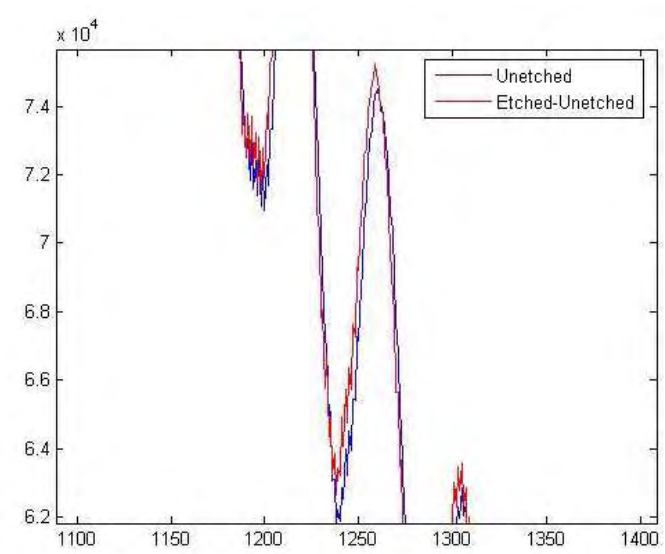

(d) Zoomed in

Figure 5.5: Intensity comparison of Interference fringes when $\mathrm{HeNe}$ light transmitted through air and etched Borofloat 


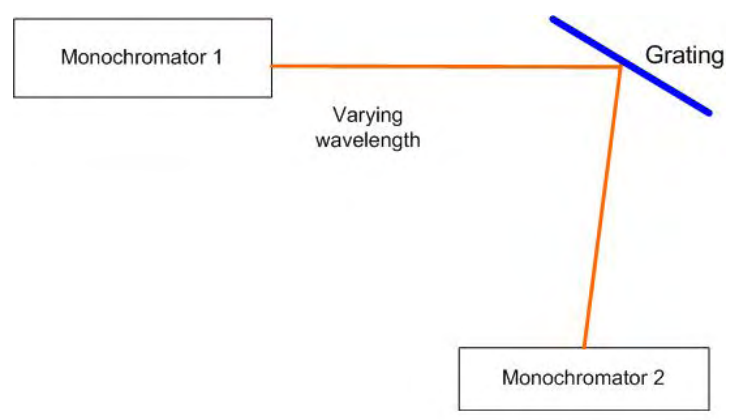

Figure 5.6: Optical set-up to vary wavelength

\subsection{Optical Characterization}

The gratings fabricated can be optically characterized by the following methods. Since, the grating fabricated by e-beam lithography and RIE in this research has a field size of $50 \mu \mathrm{m}$, a small beam spot is needed. This can be obtained as explained in section 5.3.3. Since a phasemask of $1 \mu \mathrm{m}$ period was available it was used in demonstrating the characterization methods.

\subsubsection{Intensity as a function of wavelength}

For a fixed angle of incidence and diffraction, the intensity of light diffracted off a grating will be a function of wavelength. This is demonstrated by varying the wavelength of incident light and measuring the intensity for an angle of incidence of and and angle of diffraction of for a 1 micron grating period Fig.5.4.

For red light $(\mathrm{HeNe})$, the intensity peak is seen at $633 \mathrm{~nm}$ and decreases for all other wavelengths Fig.5.7.

\subsubsection{Intensity as diffraction angle is varied}

For a fixed wavelength and angle of incidence, the intensity of light diffracted off a grating is maximum for one angle of diffraction. This is demonstrated by varying the angle of diffraction and measuring the intensity of light at each angle Fig.5.11. This was done for three different 


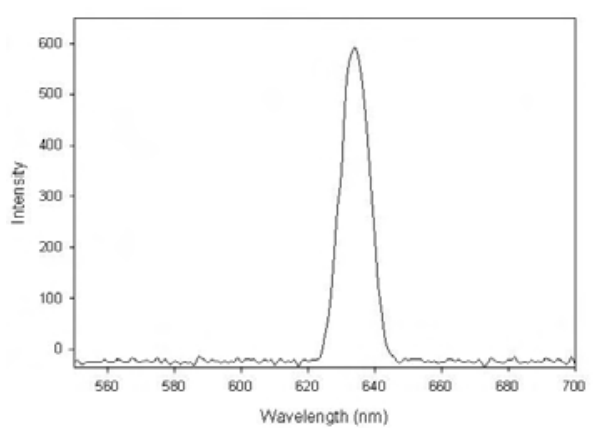

(a) $550-700 \mathrm{~nm}$

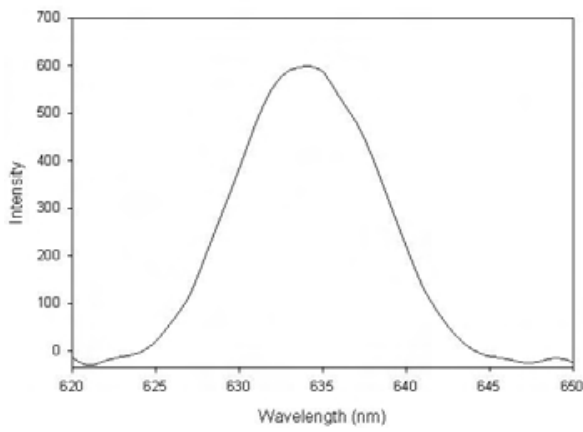

(b) $620-650 \mathrm{~nm}$

Figure 5.7: Intensity with varying wavelength

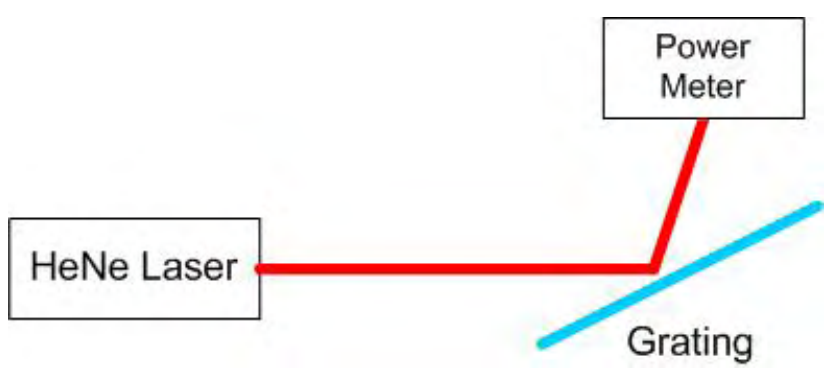

Figure 5.8: Optical set-up varying angle of diffraction

angles of incidence. The difference in measured powers may be attributed to the difference in orders of diffraction Fig.5.9.

The phasemask used was thick and the number of spots can be attributed to reflection from the rear surface. The second high intensity spot confirms this Fig.5.10. 

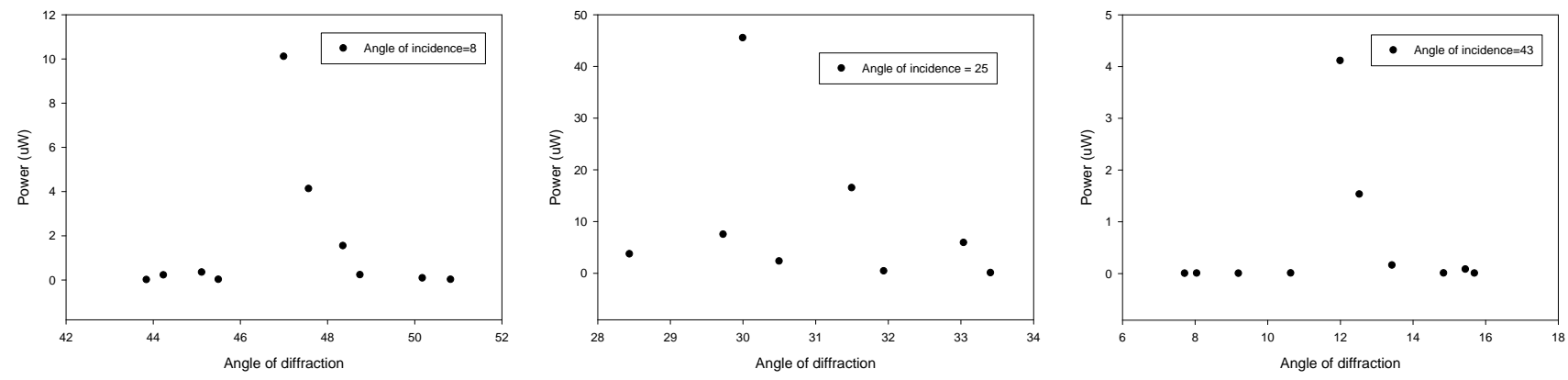

Figure 5.9: Diffraction angle measurements for three angles of incidence

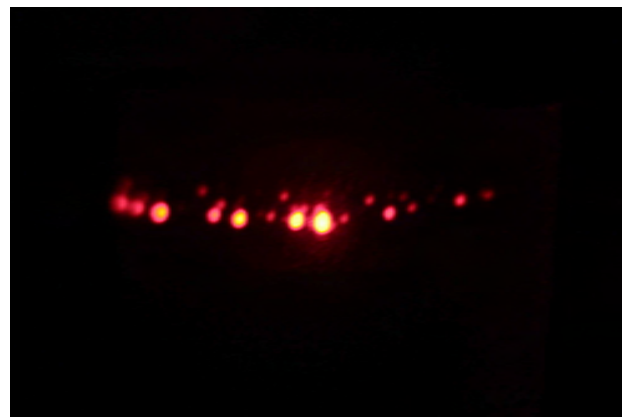

Figure 5.10: Diffraction of HeNe spot 


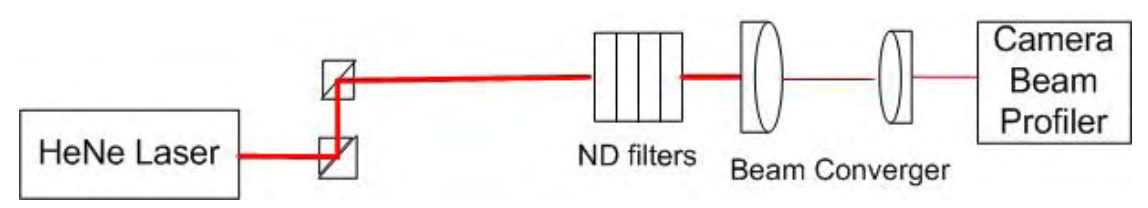

Figure 5.11: Reducing HeNe spot size

\subsubsection{Set-up to reduce spot-size for $\mathbf{5 0}$ micron gratings}

The gratings fabricated by e-beam and RIE are 50microns long. The incident beam must be smaller than the grating size. The HeNe laser emits a beam of $1 \mathrm{~mm}$ diameter. A beam profiler/camera was used to measure the beam spot size. The beam intensity was reduced using neutral density (ND) filters so as not to damage the camera sensor. A beam converger set-up using lenses is shown in Fig.5.11 that will reduce the beam spot size. Once the desired spot size is obtained, the gratings can be characterized with the optical set-ups in the previous sections. 


\section{Chapter 6}

\section{Conclusions and Future Work}

In this research the capability to fabricate grating structures in materials of interest such that they can be integrated for optical coupling has been demonstrated. The use of the CVL, both to ablate and as an exposure tool, has numerous applications. The CVL can be used to micromachine different materials including Borofloat glass. Channels $10 \mu \mathrm{m}$ wide with varying line space were ablated with a $1 \mathrm{~mm}$ aperture. By reducing the beam size, smaller lines can be ablated. Important parameters to be considered are the mask size, focus lens distance, write speed and number of passes. Using the CVL as an exposure tool with a phasemask was studied. Though it was not demonstrated in this research, relief gratings can be created in resist to be used as a mask to etch into the substrate; phase gratings (refractive index variation) can also be created in borofloat. The use of a phasemask needs optimization of fluence for both PMMA exposure, and change in refractive index of borofloat.

As show in Fig.6.1, the direct e-beam method was used to create gratings in single layer PMMA, and also in a bilayer resist, PMMA/copolymer. These were then successfully transferred to metal by lift-off and then to silicon by reactive ion etching. The ability to etch Borofloat $(\sim 125 \mathrm{~nm} / \mathrm{min})$ with vertical sidewalls at small feature sizes has been demonstrated. In addition, silica and alumina have been shown to give good etch results. The etched surface of pure silica is smooth while a slight surface roughness is seen when alumina is etched. The large percentage of metal oxides in Borofloat is shown to be responsible for its surface roughness after etch. However, since the etch residue/debris is much smaller than the wavelength of light being used, it does not 


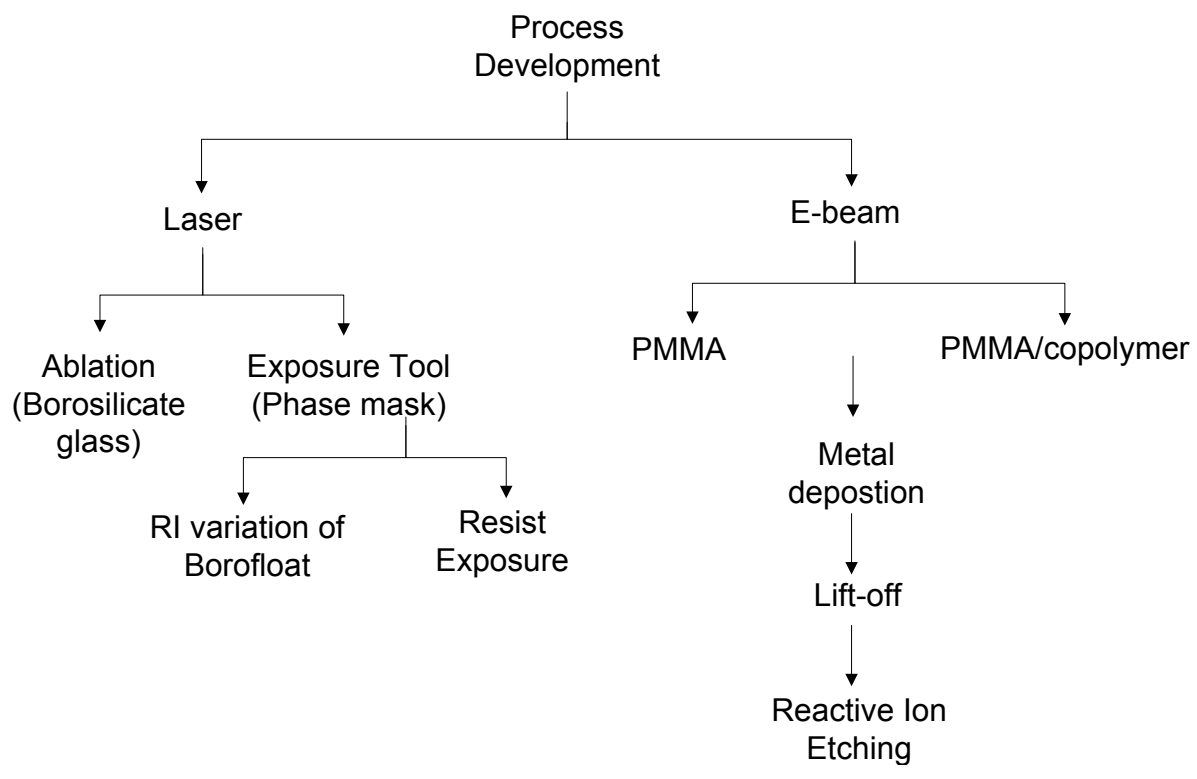

Figure 6.1: Research Process Development

significantly modify the optical properties as shown in Chapter 5.

With the ability to etch borofloat, silica and alumina, gratings can be created in any of these layers to couple light into one of the alumina waveguides in the biosensor (Fig.6.2). The laser can be used to create gratings/lenses for optical characterization of microfluidic devices in borosilicate glasses.

As a resist for e-beam lithography, a thin layer of PMMA can be used to create the grating
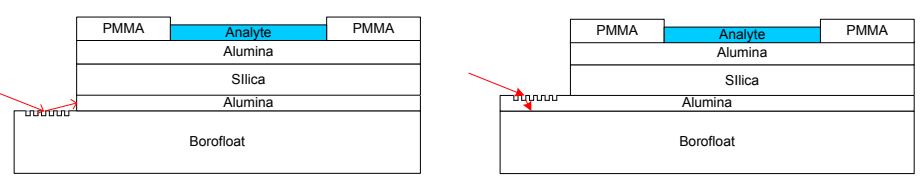

Figure 6.2: Gratings coupling light into waveguide

pattern, but may pose problems during metal deposition and lift-off. A bilayer process was successfully developed. It is necessary to use a copolymer that is much thicker than the PMMA layer above. Grating structures using a single pass of the electron beam were found to be better than the serpentine sweep for the same exposure dose. Beam spot has to be optimized for shape, focus, astigmatism, gun tilt etc. 
E-beam writing using a bilayer on silicon has been demonstrated. A similar process needs to be developed for borofloat. As Borofloat is non-conductive, the resist may charge during writing. Resists with higher molecular weights and different copolymers could be used for increased resolution. The use of a metal layer to prevent charging while writing on PMMA needs more understanding. Increasing pattern size by a method like 'stitching' in the NPGS tool should be attempted. Proximity compensation techniques can be incorporated. This will give uniform linewidths over the entire length of the pattern. The direct e-beam write can be used to create sub-micron patterns of any shape. Micro-lenses of any size can be easily fabricated. Blazed gratings in resist can be made.

After e-beam lithography, chrome is deposited and then lifted-off and is seen to be a better mask than photoresist for RIE. The etch sidewalls are improved with a metal mask. Deposition by e-beam evaporation was found to be better for lift-off than sputtering. Different masking materials for RIE can be explored. Etch rates can be increased by adding oxygen to the chamber or decreased by adding hydrogen. The chrome mask and $\mathrm{SF}_{6}$ etch recipes can be used to etch materials besides Borofloat like Si, GaN etc.

For SEM viewing of resist features, $\mathrm{Pt}$ (30nm sputtered)was found to work better than $\mathrm{Au}$. To find and view the features in borofloat in the SEM a conducting tape is necessary to ground the surface. This may be required during e-beam writing of resist on borofloat substrate. 


\section{Appendix A}

\section{A.1 Direct-write lithography}

\section{Cleaning of sample:}

Cleaning of silicon:

1. Scribe wafer to size required.

2. Clean in acetone for $5 \mathrm{~min}$. and then in methanol for $5 \mathrm{~min}$.

3. Blow dry with $\mathrm{N}_{2}$.

4. Dehydration bake in oven at $100^{\circ} \mathrm{C}$ for 30 mins.

Cleaning of borofloat:

1. Clean all containers/holders with de-I water.

2. Immerse sample in acetone for 5 mins and then in methanol for 5 mins.

3. Blow dry with $\mathrm{N}_{2}$.

4. Summa clean: Take Summa clean in a glass beaker and heat on hot plate at $50-60^{\circ} \mathrm{C}$. Turn up to 5 on hotplate and then turn down knob to 1.5 when temperature reaches $40^{\circ} \mathrm{C}$. Place sample in Summa clean once $50-60^{\circ} \mathrm{C}$ attained for 30 mins. Precaution: Use black rubber gloves when working with Summa clean in Acid hood.

\section{PMMA deposition:}

(PMMA 495 A2)

1. Place sample on spinner and spin for $30 \mathrm{sec}$. at 5000rpm. Clean with acetone and then methanol for $5 \mathrm{sec}$. each and then spin dry for the last $20 \mathrm{sec}$. (program M).

2. Using a glass pipette apply PMMA 495 A2 so as to cover the sample.

3. Slow spin at $500 \mathrm{rpm}$ for $14 \mathrm{sec}$. and at the final speed of $4000 \mathrm{rpm}$ for $55 \mathrm{sec}$. (program J).

4. Bake on hotplate at $180^{\circ} \mathrm{C}$ for $60-90$ secs.

\section{Direct Write using E-beam:}

1. Place sample in the SEM. Pump down to ultra high vacuum.

2. Note rotation of sample holder for sample to be exposed. 
3. Make sure floppy is inserted when turning on Leica machine. Turn on PC and open NPGS software. Use DesignCAD LT to create pattern file.

(a) File- $>$ New

(b) Options- $>$ Grid Settings. Set snap grid/display grid size to the dimension of the smallest feature.

(c) Use polyfill to draw.

- Set vertices for filled polygon.

- Select serpentine sweep.

- Scale need not be changed.

- All OK? - yes.

(d) Use MakeArray to repeat patterns (lines).

- Set lower left-hand corner and then upper right-hand corner.

- Enter no. of columns.

- Enter space between columns: $>0 \mu$.

- Enter no. of rows.

- Enter space between rows: $>0 \mu$.

(e) Convert to DC2 files. To do this, save file as 2D ASCII and change the name in DesignCAD LT.

4. Ensure SEM EHT voltage is $30 \mathrm{KV}$. Adjust filament current so that the electron beam spot (emission image) is as circular as possible.

5. Use copper grid to adjust focus, contrast and astigmatism.

6. Make sure beam blanker is on. Switch to software mode.

7. Open .dc2 files and set dose $\left(\mu C / \mathrm{cm}^{2}\right) /$ exposure time and save.

8. Move stage to sample and run programs to expose sample and pattern.

9. Record EHT voltage, working distance, filament current $\mathrm{I}_{f}$, probe current $\mathrm{I}_{p}$. Also record field size and time to write.

10. After writing, vent up chamber using $\mathrm{N}_{2}$ and take out sample.

\section{PMMA develop:}

1. Dip sample in MIBK/IPA 1:1 using tweezers and stir for $30 \mathrm{sec}$. to develop.

2. Then immerse in MIBK/IPA 1:3 for $30 \mathrm{sec}$. to stop developing. 
3. Blow dry with $\mathrm{N}_{2}$.

\section{Using optical microscope (Zeiss) and camera:}

1. Begin with camera at lowest magnification $4 \mathrm{X}$ and then increase to $16 \mathrm{X}$ and $40 \mathrm{X}$.

2. While taking pictures, voltage of microscope can be turned up.

3. Use polariser to obtain best contrast.

4. Turn on the camera; check battery life and make sure camera is in 'night vision' mode.

5. To take a picture, shutter for camera on the microscope column must be opened carefully. Re-focusing will be required.

6. Use remote switch to take picture. First press will cause turn on flash and second will take the picture.

7. Pictures are then exported to the PC using its USB port and Canon software.

\section{Sputtering:}

Sample is then sputtered with Pt to grow a $30-35 \mathrm{~nm}$ layer.

1. Sample mounted and then cleaned using $\mathrm{N}_{2}$. Make sure it doesn't fall when held upside down.

2. Use 'hoist up' to open chamber. Put sample above required metal target. 'Hoist down' to close the chamber.

3. Turn on Mech pump and open 'rough' valve.

4. Open cooling water tap and valve on Argon cylinder.

5. Wait till pressure gauge shows chamber pressure is down to 1. Turn off Mech pump and turn on HVAC.

6. The chamber is now open to the cryo pump and is further pumping down. When pressure is down to last 2 decimal places, turn on Ion guage and wait till pressure goes down to $5 \times 10^{-6}$.

7. Turn on the power to the magnetron drive.

8. Set timer for deposition time.

9. Close the throttle valve and turn on Gas1.

10. Ar flow should stabilize around 30 and pressure around $60 \mathrm{mT}$. 
11. Turn on the DC Target selector and select Station 4 (US gun).

12. Turn on power supply. Deposition power is set at $0.1 \mathrm{KW}$. Let timer count down $1 \mathrm{~min}$ and note stable voltage $(\mathrm{V}=436)$. Sputter for $1 \mathrm{~min}$ with lid/shutter on (pre-deposition). After the target is clean, turn on timer and shutter (4) at the same time so that the sample is now sputtered with Pt for $30 \mathrm{sec}$.

13. When timer runs out, system shuts off automatically. Turn off power and Gas 1 and open throttle valve. Close Ar (cylinder).

14. Turn off HVAC and hit VENT. When green VACUUM light turns off and pressure on gauge (left) goes up (261-283), 'hoist up' to open the chamber. A hissing sound is heard caused by $\mathrm{N}_{2}$. Turn off VENT.

15. Remove sample.

\section{RIE:}

1. Make sure System On and Pump On switches are on.

2. Start Turbo Pump.

3. Open PcPlus software. Login as Installation Engineer. In 'manual' mode choices of status and vacuum are available. In Status mode, the processes such as pumping to base pressure, changing gas flows, powers, pressure or venting can be done manually. The 'display' mode has options of Status/Vacuum/Process. Display mode is to be used when running pre-written programs. The vacuum option in both modes gives the same information as status mode but in a diagramatic manner. The third mode 'edit' allows programs to be written or changed.

4. Write program for etch.

5. File is to be run from 'display' mode by selecting it and then 'start'.

6. Base pressure used is $5 \times 10^{-6}$ mbar to clean the chamber of any unwanted gas.

7. Once base pressure is attained, valves open and gas flows into the chamber. After the flow stabilizes, the pressure of the chamber is increased to the process pressure. The power supplies turn on and a plasma is struck.

8. Oxygen descum is used to remove residual HMDS/copolymer left after development (30 sccm $\mathrm{O}_{2}$ at $300 \mathrm{mT}, 150 \mathrm{~W}$ RF for $30 \mathrm{sec}$.).

9. To strip the photoresist after RIE of borofloat, $30 \mathrm{sccm}$ of $\mathrm{O}_{2}$ is used at $300 \mathrm{mT}, 150 \mathrm{~W}$ RF for $12 \mathrm{~min}$. 


\section{A.2 Photolithography:}

Used to create samples for RIE.

Substrate: Borofloat

Positive Process:

- Clean with acetone, methanol, dry $\mathrm{N}_{2}$

- Dehydration bake 30 mins. at $100^{\circ} \mathrm{C}$

- HMDS primer (adhesion promoter)

- Spin on resist AZ 5214 at $5500 \mathrm{rpm}$ for 55 secs. $(1.4 \mu-1.6 \mu)$

- Soft bake on hot plate $90^{\circ} \mathrm{C}$ for 60 secs.

- Expose in Karl Suss MA6 for $55 \mathrm{secs}$ at $2.4 \mathrm{~mW} / \mathrm{cm}^{2}\left(77.7 \mathrm{secs}\right.$ at $\left.1.7 \mathrm{~mW} / \mathrm{cm}^{2}\right)$

- Develop AZ312MIF and D-I water (1:1.2) for 100 secs.

Image reversal process:

- Clean with acetone, methanol, dry $\mathrm{N}_{2}$

- Dehydration bake 30 mins. at $100^{\circ} \mathrm{C}$

- HMDS primer (adhesion promoter)

- Spin on resist AZ 5214 at $5500 \mathrm{rpm}$ for 55 secs. $(1.4 \mu-1.6 \mu)$

- Soft bake on hot plate $90^{\circ} \mathrm{C}$ for 60 secs.

- Expose in Karl Suss MA6 for $15 \mathrm{secs}$ at $2.5 \mathrm{~mW} / \mathrm{cm}^{2}\left(37.5 \mathrm{~mJ} / \mathrm{cm}^{2}\right)$

- Post bake at $120^{\circ} \mathrm{C}$ for 2 mins.

- Flood expose for 82 secs.

- Develop AZ 400K and D-I water (1:6) for 100 secs.

- Hard bake at $100^{\circ} \mathrm{C}$ for 30 mins.

Substrate: SiO2 (oxide wafers)

Positive Process:

- Clean with acetone, methanol, dry $\mathrm{N}_{2}$

- Dehydration bake 30 mins. at $100^{\circ} \mathrm{C}$ 
- HMDS primer (adhesion promoter)

- Spin on resist AZ 5214 at $5500 \mathrm{rpm}$ for 55 secs. $(1.4 \mu-1.6 \mu)$

- Soft bake on hot plate $90^{\circ} \mathrm{C}$ for 60 secs.

- Expose in Karl Suss MA6 for 50 secs at $1.8 \mathrm{~mW} / \mathrm{cm}^{2}\left(77.7 \mathrm{secs}\right.$ at $\left.1.7 \mathrm{~mW} / \mathrm{cm}^{2}\right)$

- Develop AZ312MIF and D-I water (1:1.2) for 100-120 secs. 


\section{Appendix B}

\section{B.1 Spin programs:}

Clean: Program M:

$5000 \mathrm{rpm}: 30 \mathrm{sec}$.

PMMA - thinnest layer 70nm Program J:

1. $500 \mathrm{rpm}$ for $14 \mathrm{sec} .(9+5)$ i.e. $9 \mathrm{sec}$ to ramp up and $5 \mathrm{sec}$ at $500 \mathrm{rpm}$.

2. $4000 \mathrm{rpm}$ for $55 \mathrm{sec} .(10+45)$ i.e. $10 \mathrm{sec}$ to ramp up and $45 \mathrm{sec}$ at $4000 \mathrm{rpm}$.

Copolymer - thick layer 400nm Program H:

1. $500 \mathrm{rpm}$ for $14 \mathrm{sec} .(9+5)$ i.e. $9 \mathrm{sec}$ to ramp up and $5 \mathrm{sec}$ at $500 \mathrm{rpm}$.

2. $1000 \mathrm{rpm}$ for $55 \mathrm{sec}$. $(10+45)$ i.e. $10 \mathrm{sec}$ to ramp up and $45 \mathrm{sec}$ at $1000 \mathrm{rpm}$.

\section{B.2 Sputtering:}

Target: Pt

Vacuum Pressure: $5 \times 10^{-6}$ mbar

Argon: Pressure: $60 \mathrm{mT}$ Flow: $30 \mathrm{sccm}$

Target voltage: $432 \mathrm{~V}$

Deposition Power: 0.1KW

Time: Pre-deposition: 1min Deposition: 0.5min (30-35nm)

Target: $\mathrm{Cr}$

Vacuum Pressure: $5 \times 10^{-6} \mathrm{mbar}$

Argon: Pressure: $60.1 \mathrm{mT}$ Flow: $30.1 \mathrm{sccm}$

Target Voltage: $441 \mathrm{~V}$

Power: $0.1 \mathrm{KW}$

Time: Pre-deposition: 1 min Deposition: 3 min. (120nm)

\section{B.3 RIE sample recipe}

1. Base pressure: $5 \times 10^{-6}$ mbar

2. Process:
(a) $\mathrm{O}_{2}: 30 \mathrm{sccm}$
(b) RF forward power: $150 \mathrm{~W}$
(c) Process pressure: $0.03 \mathrm{~T}$
(d) Time: $30 \mathrm{sec}$.

3. Action: Chamber Vent. 


\section{Appendix C}

\section{SEM Images of 4.1.2}

An exposure between $85-87 \mu \mathrm{C} / \mathrm{cm}^{2}$ would be best. There is a slight distortion seen at the start

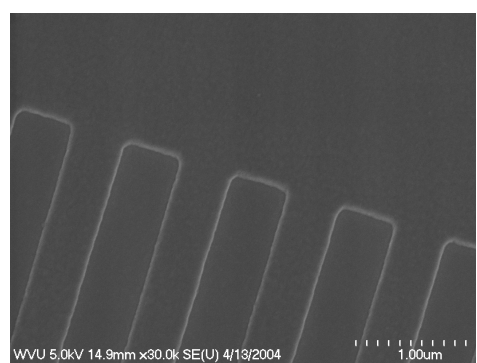

(a) Edge of pattern

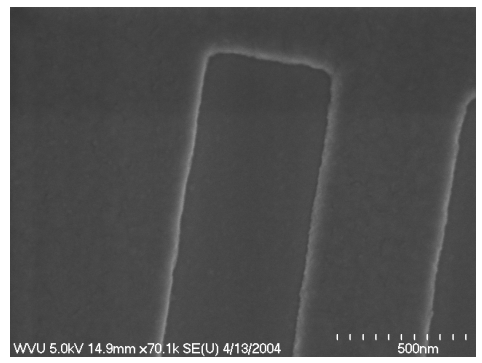

(d) Trough $=494 \mathrm{~nm}$ Ridge $=517 \mathrm{~nm}$

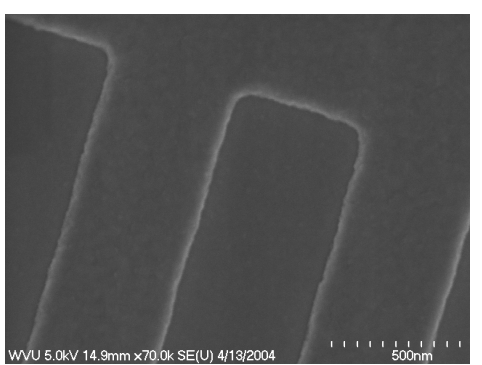

(b) trough $=520 \mathrm{~nm}$ ridge $=509 \mathrm{~nm}$ $90 \mu \mathrm{C} / \mathrm{cm}^{2}$ exposure

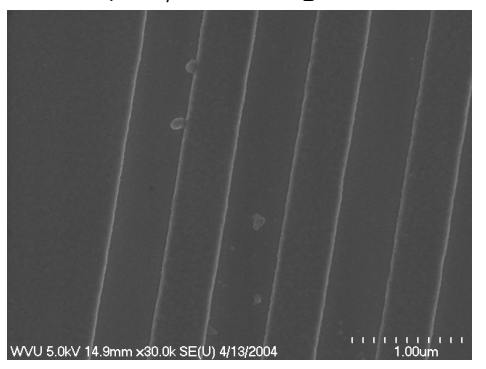

(e) Center of pattern $80 \mu \mathrm{C} / \mathrm{cm}^{2}$ exposure

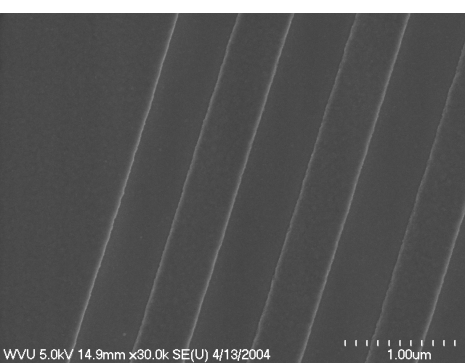

(c) Center of pattern

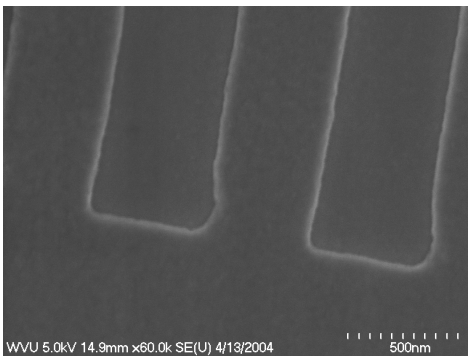

(f) Start of pattern

Figure 6.3: $0.5 \mu$ lines

of the lines (Fig.1 (f)). This could be due to the beam blanker not turning off when it moves from one line to another. 


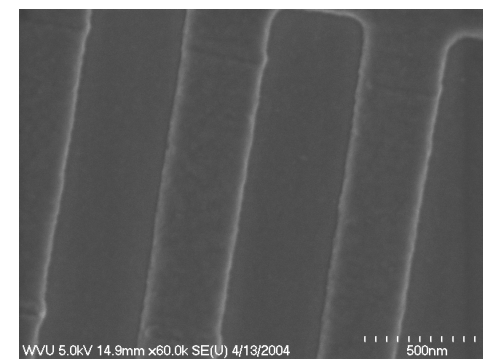

(a) $90 \mu \mathrm{C} / \mathrm{cm}^{2}$ exposure Trough $=433 \mathrm{~nm}$ ridge $=381 \mathrm{~nm}$

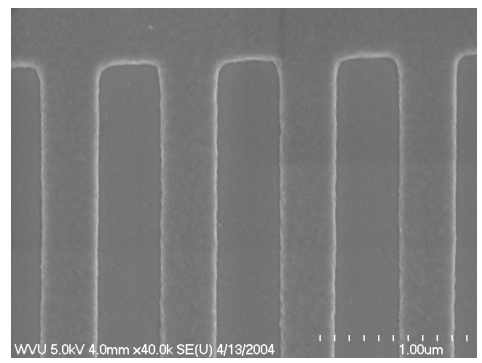

(b) $80 \mu \mathrm{C} / \mathrm{cm}^{2}$ exposure Trough $=429 \mathrm{~nm}$ ridge $=382 \mathrm{~nm}$

Figure 6.4: $0.4 \mu$ lines

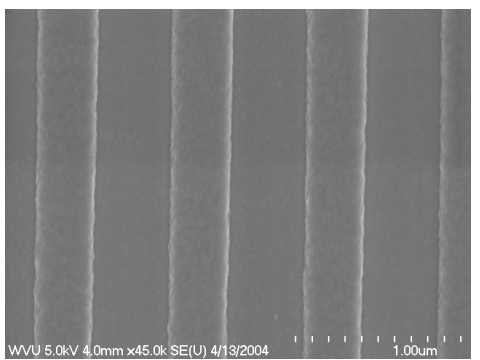

(c) $80 \mu \mathrm{C} / \mathrm{cm}^{2}$ exposure Center of pattern

$90 \mu \mathrm{C} / \mathrm{cm}^{2}$ seems to be too much exposure for $0.4 \mu$ lines.

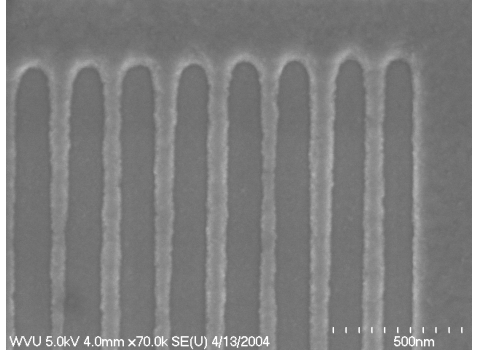

(a) $90 \mu \mathrm{C} / \mathrm{cm}^{2}$ exposure Trough $=125 \mathrm{~nm}$ ridge $=71-96 \mathrm{~nm}$

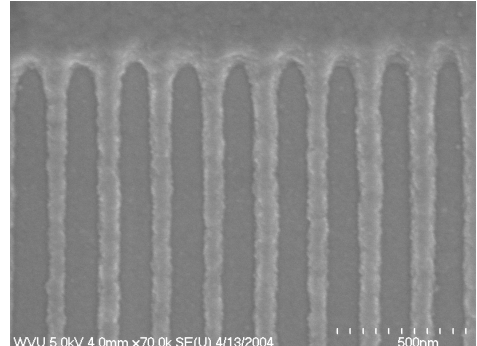

(b) $80 \mu \mathrm{C} / \mathrm{cm}^{2}$ exposure

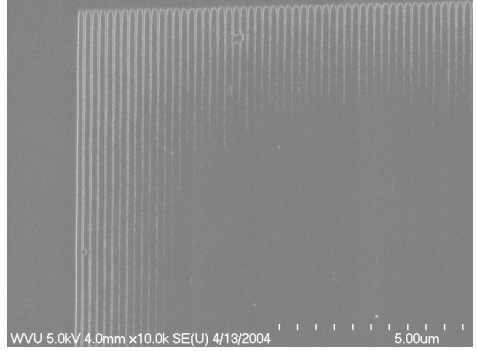

(c) $80 \mu \mathrm{C} / \mathrm{cm}^{2}$ exposure Pattern when zoomed out

Figure 6.5: $0.1 \mu$ lines

Lines disappear at the center of the patterns. Exposure needs to be reduced. 


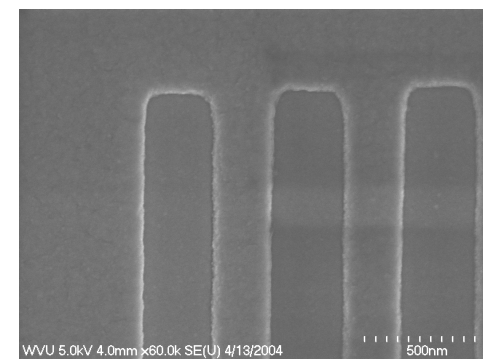

(a) $90 \mu \mathrm{C} / \mathrm{cm}^{2}$ exposure Trough $=311 \mathrm{~nm}$ ridge $=266 \mathrm{~nm}$

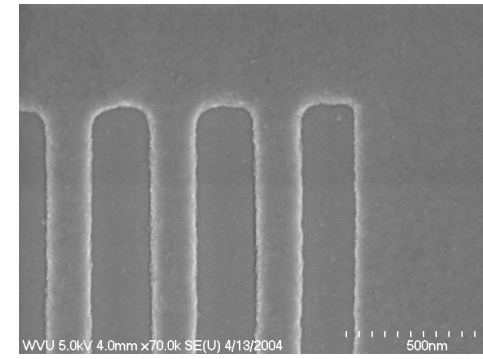

(a) $90 \mu \mathrm{C} / \mathrm{cm}^{2}$ exposure Trough $=223 \mathrm{~nm}$ ridge $=179 \mathrm{~nm}$

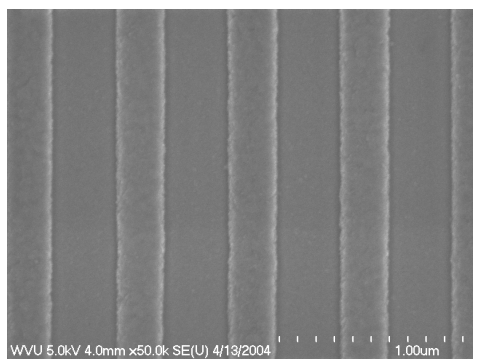

(b) $80 \mu \mathrm{C} / \mathrm{cm}^{2}$ exposure Trough $=347 \mathrm{~nm}$ ridge $=258 \mathrm{~nm}$ $0.3 \mu$ lines

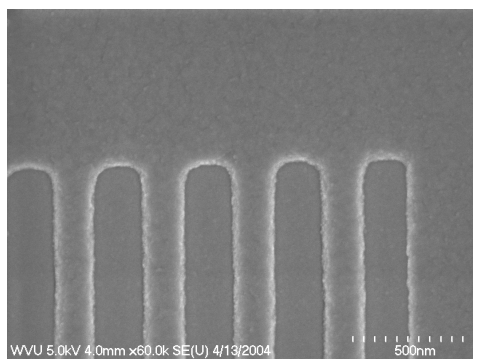

(b) $80 \mu \mathrm{C} / \mathrm{cm}^{2}$ exposure Trough $=218 \mathrm{~nm}$ ridge $=185 \mathrm{~nm}$ $0.2 \mu$ lines

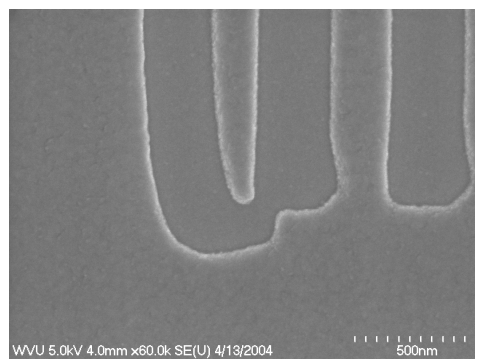

(c) $80 \mu \mathrm{C} / \mathrm{cm}^{2}$ exposure Distortion

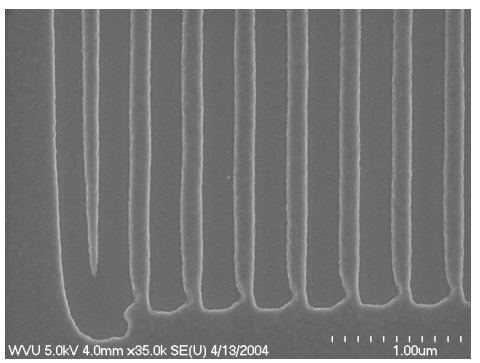

(c) $80 \mu \mathrm{C} / \mathrm{cm}^{2}$ exposure Distortion

Figure 6.6: 0.3 and $0.2 \mu$ lines

The lines have merged at the start of the write. Doses are too high as seen from pictures and measurements. Even $80 \mu \mathrm{C} / \mathrm{cm}^{2}$ seems a little too high for the $0.2 \mu$ feature. The trough is wider than the ridge. As the features get smaller, the exposure should be lowered. 


\section{SEM Images of 4.1.3.1}

Lines are merged at the start of pattern. Distinct lines are not seen. They do not run the whole length.

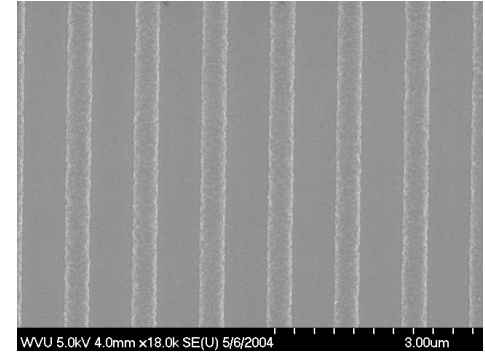

(a) $0.5 \mu$ lines at $87 \mu \mathrm{C} / \mathrm{cm}^{2}$ Trough $=639 \mathrm{~nm}$ Ridge $=364 \mathrm{~nm}$

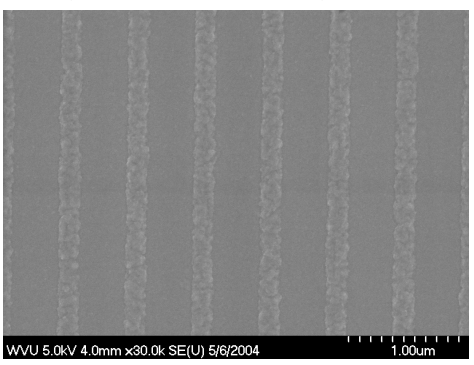

(d) $0.2 \mu$ lines at $75 \mu \mathrm{C} / \mathrm{cm}^{2}$ Trough $=410 \mathrm{~nm}$ Ridge $=175 \mathrm{~nm}$

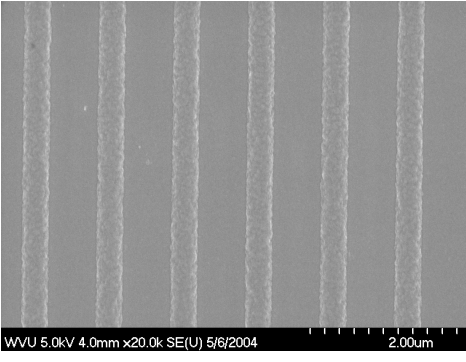

$0.4 \mu$ lines at $80 \mu \mathrm{C} / \mathrm{cm}^{2}$

Trough $=665 \mathrm{~nm}$ Ridge $=357 \mathrm{~nm}$

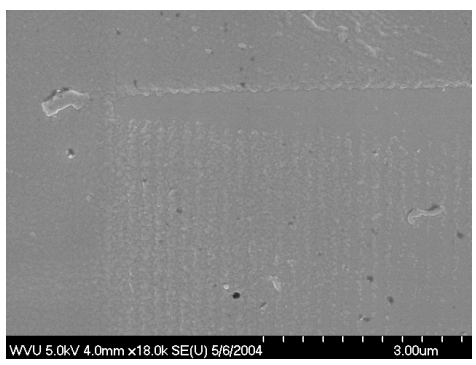

(e) $0.2 \mu$ lines at $60-75 \mu \mathrm{C} / \mathrm{cm}^{2}$

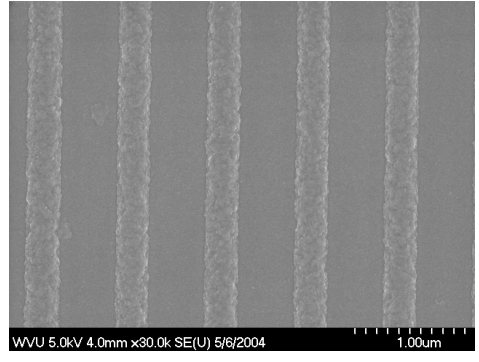

$0.3 \mu$ lines at $77 \mu \mathrm{C} / \mathrm{cm}^{2}$ Trough $=516 \mathrm{~nm}$ Ridge $=291 \mathrm{~nm}$

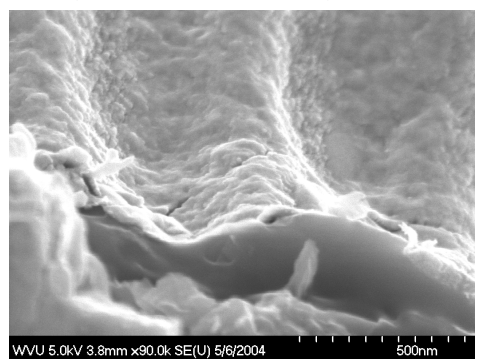

(f)Edge of $0.4 \mu$ lines at $80 \mathrm{uC} / \mathrm{cm} 2$ Bad cross-sectioning

Figure 6.7: 0.1-0.5 $\mu$ lines in PMMA at different exposures

Center of the square is not there at all. With lower exposures, lines seem to have been attempted but they are not distinct. The PMMA hasn't been exposed all the way to the bottom. 


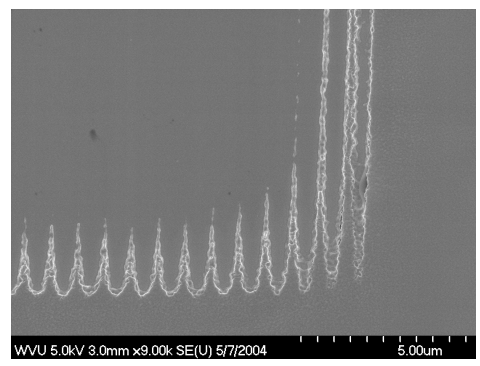

(a)

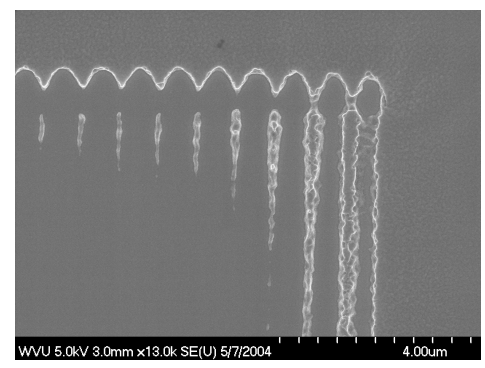

(b)

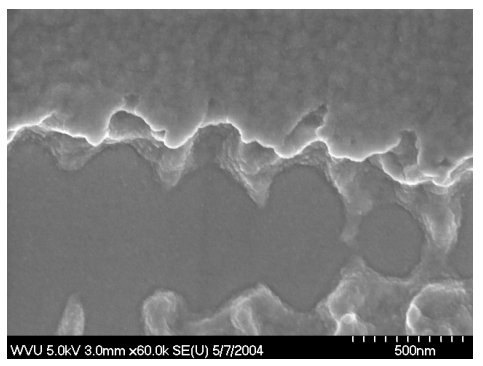

(c)

Figure 6.8: $0.4 \mu$ lines at $80 \mu \mathrm{C} / \mathrm{cm}^{2}$ in PMMA/copolymer

Patterns of $0.2,0.3$ and $0.5 \mu$ are similar. Lines are overexposed. PMMA and copolymer are not exposed/developed the same (Fig.6(c)). $0.1 \mu$ lines were not formed, but a surface roughness was seen. 


\section{SEM Images of 4.1.3.3}

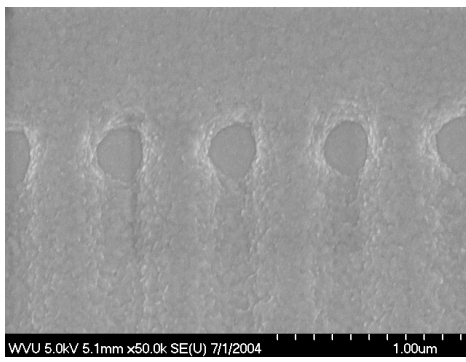

(a) $70 \mu \mathrm{C} / \mathrm{cm}^{2}$ $0.3 \mu$ Serpentine sweep

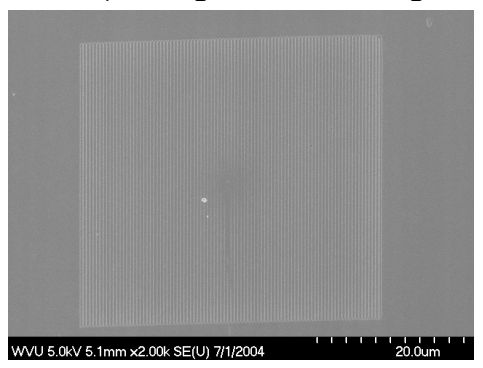

(c) $0.3 \mu 100 \mu \mathrm{C} / \mathrm{cm}^{2}$

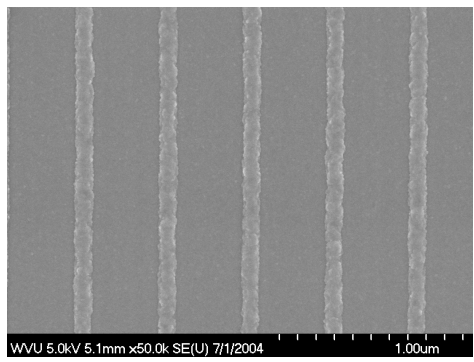

(b) Trough $=353 \mathrm{~nm}$ Ridge $=91 \mathrm{~nm} 120 \mu \mathrm{C} / \mathrm{cm}^{2}$

$0.3 \mu$ Single pass

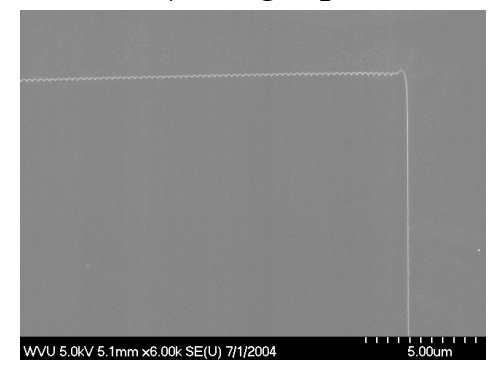

(d) $0.2 \mu 100 \mu \mathrm{C} / \mathrm{cm}^{2}$

Figure 6.9: PMMA

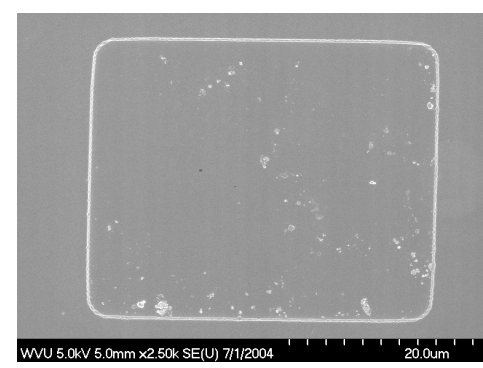

Figure 6.10: Single pass writes on PMMA/copolymer sample

All single pass write on the PMMA/copolymer samples resulted in completely exposed squares. 


\section{Bibliography}

[1] D. F. Kyser and N. S. Viswanathan, "Monte carlo simulation of spatially distributed beams in electron-beam lithography," Journal of Vacuum Science Technology, vol. 12, no. 6, pp. 1305-1308, 1975 .

[2] J.M. Dawson, Integrated Through-Wafer Optical Monitoring of MEMS for Closed-Loop Control, Ph.D. thesis, West Virginia University, 2002.

[3] Donald Lloyd, Lawrence Hornak, Shantanu Pathak, Dale Morton, and Ian Stevenson, "SPARROW A new thin film based biosensor chip technology," Vacuum Technology and Coating, September 2004.

[4] Shantanu Pathak, "Resonant optical waveguide biosensor characterization," M.S. thesis, West Virginia University, Dec. 2004.

[5] Stephen A. Campbell, The Science and Engineering of Microfabrication, Oxford University Press.

[6] J.M Stauffer, Y. Oppliger, and P. Renault, "Electron beam writing of continuous resist profiles for optical applications," Journal of Vacuum Science and Technology, vol. B, no. 10, pp. 6, Nov/Dec 1992.

[7] M Ekberg, F. Nikolajeff, M. Larsson, and S. Hard, "Proximity-compensated blazed transmission grating manufacture with direct-writing, electron-beam lithography.," Applied Optics, vol. 33, no. 1, Jan 1994.

[8] H.C. Pfeiffer, "Variable spot shaping for electron beam lithography," Journal of Vacuum Science Technology, , no. 15, pp. 887, 1978.

[9] Mihir Parikh, "Self-consistent proximity effect correction technique for resist exposure (SPECTRE)," Journal of Vacuum Science and Technology, vol. 15, no. 3, May/June 1978.

[10] Mihir Parikh, "Proximity effects in electron lithography: Magnitude and correction techniques," IBM Journal of Resist Development, vol. 24, no. 4, July 1980.

[11] W.D. Grobman, A.J. Speth, and T.H.P. Chang, "Proximity correction enhancements for 1- $\mu$ m dense circuits," IBM Journal of Resist Development, vol. 24, no. 5, Sept. 1980.

[12] M Pekerar, R Bass, and K-W Rhee, "Sub-0.1u electron-beam lithography for nanostructure development," Journal of Vacuum Science Technology B, vol. 18, no. 6, Nov/Dec 2000. 
[13] R. E. Howard, E.L. Hu, L.D. Jackel, P. Grabbe, and D.M. Tennant, "400- $\dot{A}$ linewidth e-beam lithography on thick silicon substrates," Applied Physics Letters, vol. 36, no. 7, April 1980.

[14] Raman Kashyap, Fiber Bragg Gratings, Marcel Dekker, Inc., 1997.

[15] E.G Lowen and E.Popov, Diffraction Gratings and Applications.

[16] S.Y Chou and P.B. Fischer, "Double 15-nm-wide metal gates 10nm apart and $70 \mathrm{~nm}$ thick on GaAs," Journal of Vacuum Science Technology B, vol. 8, no. 6, Nov/Dec 1990.

[17] P.B. Fischer and S.Y. Chou, "Sub-50 nm high aspect-ratio silicon pillars, ridges, and trenched fabricated using ultrahigh resolution electron beam lithography and reactive ion etching," Applied Physics Letters, vol. 62, no. 12, March 1993.

[18] H. Nishihara, Y. Handa, V. Suhara, and J. Koyama, "Direct writing of optical gratings using a scanning electron microscope," Applied Optics, vol. 17, no. 15, Aug 1978.

[19] J. H. Magerlein and D. J. Webb, "Electron-beam resists for lift-off processing with potential application to Josephson integrated circuits," Sept 1980.

[20] M. Hatzakis, "PMMA copolymers as high sensitivity electron resists," Journal of Vacuum Science Technology, vol. 16, no. 6, pp. 1984-1988, 1979.

[21] R. E. Howard, E.L. Hu, and L.D. Jackel, "Multilevel resist for lithography below 100nm," IEEE Transactions on Electron Devices, vol. ED-28, no. 11, Nov. 1980.

[22] W.D. Grobman, T.P. Donahue, M. Hatzakis, H.E. Luhn, A. Wilson, T.H.P. Chang, and A.J. Speth, “1 $\mu \mathrm{m}$ MOSFET VLSI technology- part VI electron-beam lithography," IEEE Journal of Solid State Circuits, vol. SC-14, no. 2, April 1979.

[23] Y. Chen, D. Macintyre, and S. Thoms, "Electron beam lithography processes for T- and $\gamma$ - shaped gate fabrication using chemically amplified DUV resists and PMMA," Journal of Vacuum Science Technology, vol. B17, no. 6, Nov/Dec 1999.

[24] Kirt R. Williams and Richard S. Muller, "Etch rates for micromachining processing," Journal of Microelectromechanical Systems, vol. 5, no. 4, Dec 1996.

[25] Kirt R. Williams, Kishan Gupta, and Richard S. Muller, "Etch rates for micromachining processingII," Journal of Microelectromechanical Systems, vol. 5, no. 4, Dec 1996.

[26] M. Esashi X. Li, T. Abe, "Deep reactive ion etching of PyREX glass using $\mathrm{SF}_{6}$ plasma," Sensors and Actuators A, vol. 87, no. 3, pp. 139-145, 2001.

[27] K. Venkatakrishnan, N. R. Sivakumar, and B. Tan, "Fabrication of planar gratings by direct ablation using an ultrashort pulse laser in a common optical path configuration," Applied Physics A, vol. 76, 2003.

[28] C.J.Paddison, J.M. Dawes, D.J.W. Brown, M.J. Withford, and R.I. Trickett, "Multiple fibre gratings fabricated using frequency-doubled copper vapor lasers," Electronics Letters, , no. 34, pp. 25, 1998. 
[29] H.J. Booth, E.K. Illy, M.R.H Knowles, G.T. Purves, and A.J Kearsley, "Manufacture of photonics components with a deep UV laser source at 255nm," ICALEO Proceedings, 2001.

[30] L.Zhang, Y.Liu, and I.Bennion, "High reflectivity single and multiple-grating fabrication of bragg and long-period structures using $255 \mathrm{~nm}$ UV light from a frequency-doubled copper vapor laser," Proc. Int. Conf. on Bragg Gratings, Photosensitivity, and Poling in Glass Waveguides, Sept. 1999.

[31] V. Mizrahi, P.J. Lemaire, T Ergodan, W. A. Reed, D. J. DiGiovanni, and R.M. Atkins, "Ultraviolet laser fabrication of ultrastrong optical fiber gratings and of germania-doped channel waveguides," Applied Physics Letters, , no. 63, pp. 13, Sept. 1993.

[32] C-Y Chao, C-Y Chen, C-W Liu, Y. Chang, and .C.C. Yang, "Direct writing of silicon gratings with highly coherent ultraviolet laser," Applied Physics Letters, vol. 71, no. 17, Oct. 1997.

[33] B.J. Eggleton, P.A.Krug, and L. Poladian, "Experimental demonstration of compression of dispersed optical pulses by reflection from self-chirped optical fiber bragg gratings," Optics Letters, vol. 19, no. 12, June 1994.

[34] K.O.Hill, B. Malo, F. Bilodeau, D.C. Johnson, and J.Albert, "Bragg gratings fabricated in monomode photosensitive optical fiber by UV exposure through a phase mask," Applied Physics Letters, vol. 62, no. 10, March 1993.

[35] W. Hinsberg, F.A. Houle, J. Hoffnagle, M. Sanchez, G. Walraff, M. Morrison, and S. Frank, "Deepultraviolet interferometric lithography as a tool for assessment of chemically amplified photoresist performance," Journal of Vacuum Science Technology B, vol. 16, no. 6, Nov/Dec 1998.

[36] J.Y. Decker, A. Fernandez, and D.W. Sweeney, "Generation of subquarter-micron resist structures using optical interference lithography and image reversal," Journal of Vacuum Science Technology $B$, vol. 15, no. 6, Nov/Dec 1997.

[37] G. Sepold C. Wochnowski, S. Metev, "UV-laser-assisted modification of the optical properties of polymethylmethacrylate,” Applied Surface Science, vol. 154-155, pp. 706-711, 2000.

[38] S. Caulder P.J. Scully and R Bartlett, "UV-laser photo-induced refractive index changes in poly methyl methacrylate and plastic optical fibers for application as sensors and devices," Science-Laser for Science Facility-Physics, 1999/2000.

[39] Anna Massimi, Enzo Di Fabrizio, Massimo Gentili, Davide Piccinin, and Mario Matinelli, "Fabrication of optical wave-guides in silica-on-silicon by nickel electroplating and conventional reactive ion etching," Japanese Journal of Applied Physics, vol. 38, no. 10, pp. 6150-6153, Oct 1999. 


\section{PRODUCT ATTRIBUTES}

- Submicron linewidth control

- Sub $0.1 \mu \mathrm{m}$ imaging

- E-beam, X-ray \& deep UV imaging

- Broad range of molecular weights \& dilutions

- Excellent adhesion to most substrates

- Compatible with multi-layer processes

\section{APPLICATIONS}

- Multi-layer T-gate processing

- Direct write e-beam lithography

- Protective coatings for wafer thinning

- Adhesive for X-ray LIGA processing

- Sacrificial layers

\section{$\mathrm{NANO}^{\mathrm{TM}}$ PMMA and Copolymer}

PMMA (polymethyl methacrylate) is a versatile polymeric material that is well suited for many imaging and non-imaging microelectronic applications. PMMA is most commonly used as a high resolution positive resist for direct write e-beam as well as $\mathrm{x}$-ray and deep UV microlithographic processes. PMMA is also used as a protective coating for wafer thinning, as a bonding adhesive and as a sacrificial layer.

Standard PMMA products cover a wide range of film thicknesses and are formulated with 495,000 \& 950,000 molecular weight (MW) resins in either chlorobenzene or the safer solvent anisole. Custom MW products ranging from 50,000 - 2.2 million are available upon request. In addition, we offer copolymer (MMA (8.5) MAA) products formulated in the safer solvent ethyl lactate. All MCC PMMA and copolymer resists are available in package sizes from $500 \mathrm{ml}$ to 20 liters.

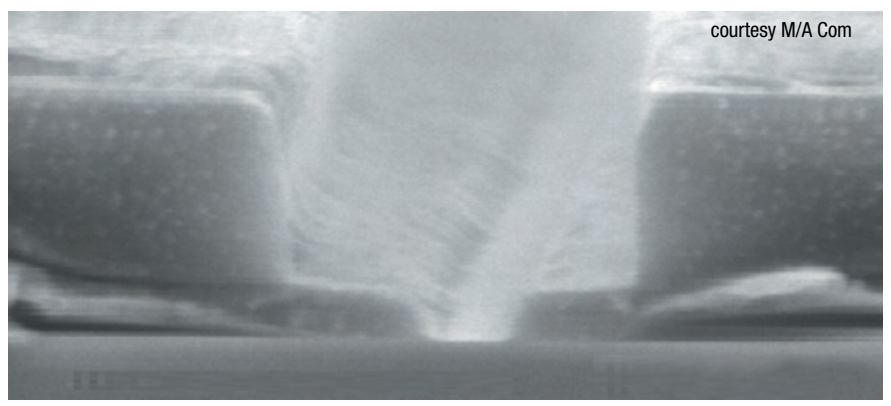

100nm gate profile imaged in 495K PMMA with 8.5 MAA Copolymer on top.

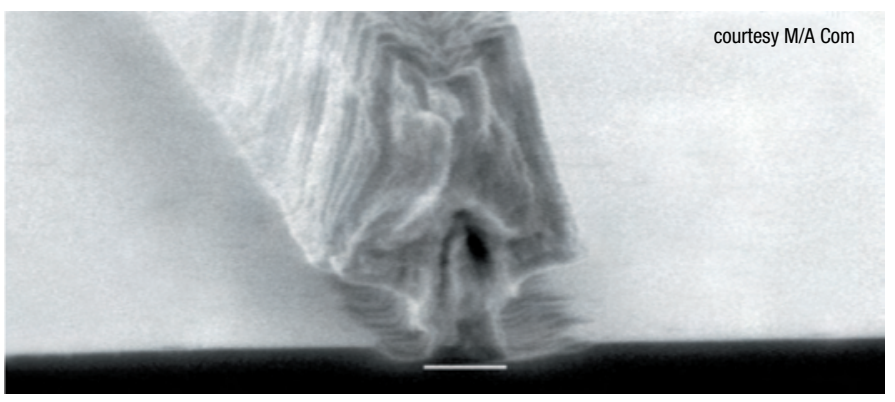

T-gate resulting from PMMA/Copolymer bilayer resist stack. 


\section{PROCESSING GUIDELINES}

\section{Substrate Preparation}

The substrate should be clean and dry. Solvent, $\mathrm{O}_{2}$ plasma, and $\mathrm{O}_{3}$ cleans are commonly used and recommended.

Coat

MicroChem PMMA resists produce low defect coatings over a broad range of film thicknesses. The film thickness vs. spin-speed curves displayed in Fig. 1 through 8 provide the information required to select the appropriate PMMA dilution and spin speed needed to achieve the desired film thickness.

The recommended coating conditions are:
(1) Dispense: STATIC 5 - $8 \mathrm{ml}$ for a $150 \mathrm{~mm}$ wafer
(2) Spread: DYNAMIC $500 \mathrm{rpm}$ for $5 \mathrm{sec}$ OR STATIC $0 \mathrm{rpm}$ for $10 \mathrm{sec}$

(3) Spin: $\quad$ Ramp to final spin speed at a high acceleration rate and hold for a total of 45 seconds.

\section{Pre Bake}

\section{PMMA}

Hot plate:

$180^{\circ} \mathrm{C}$ for $60-90 \mathrm{sec} 0 \mathrm{R}$

Convection Oven: $\quad 170^{\circ} \mathrm{C}$ for $30 \mathrm{~min}$

\section{Copolymer}

Hot plate: $150^{\circ} \mathrm{C}$ for $60-90 \mathrm{sec} 0 \mathrm{R}$

Convection Oven: $\quad 140^{\circ} \mathrm{C}$ for $30 \mathrm{~min}$

*Vacuum oven bake can also be used
Typical process flow for bi-layer T-gate process
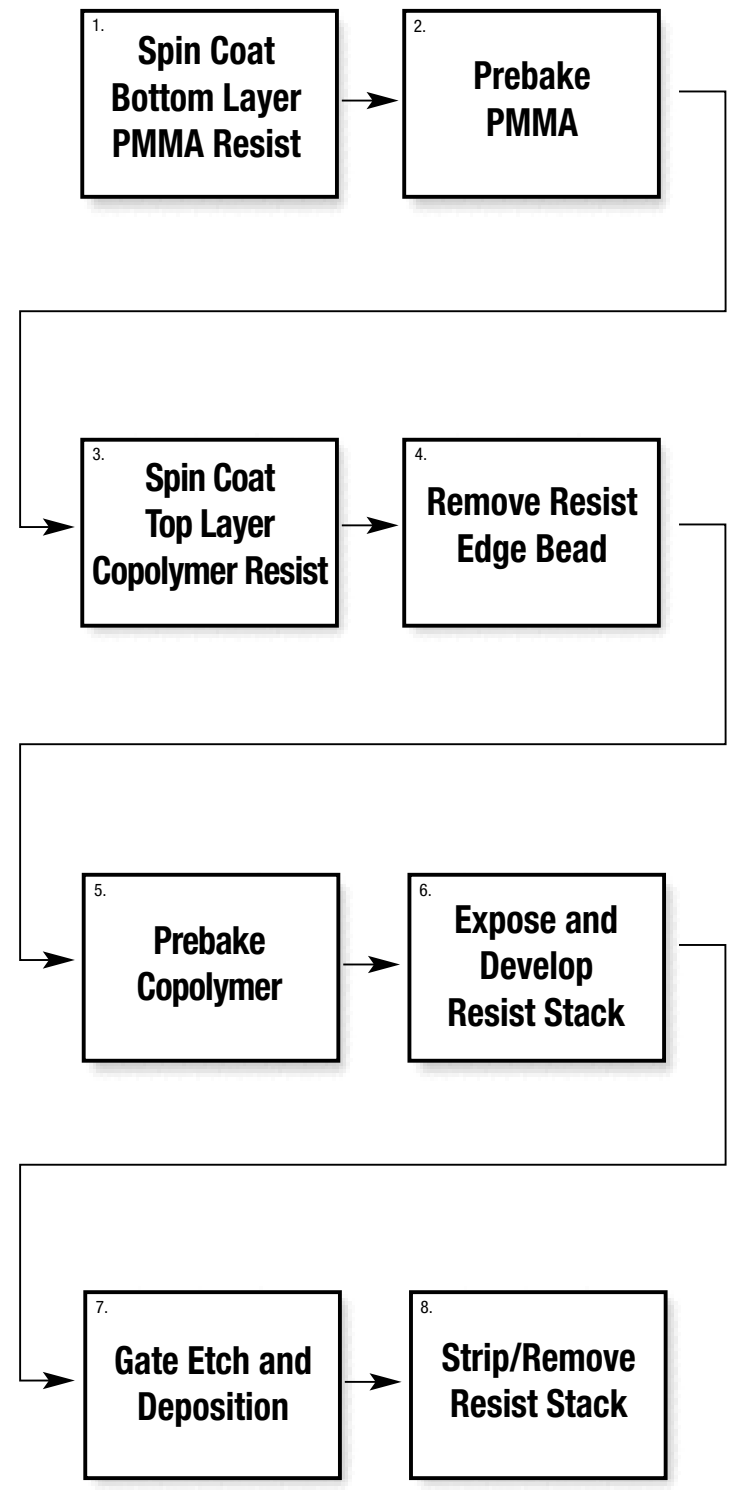


\section{Expose}

PMMA can be exposed with various parts of the electromagnetic spectrum.

e-beam: Dose - $50-500 \mu \mathrm{C} / \mathrm{cm}^{2}$ depending on radiation source/equipment \& developer used.

Energy - 20-50kV; higher kV for higher resolution, e.g. 50kV for $0.1 \mathrm{~mm}$ images.

DUV(deep UV): Low sensitivity, requiring doses $>500 \mathrm{~mJ} / \mathrm{cm}^{2}$ at $248 \mathrm{~nm}$.

X-ray: Sensitivity of PMMA is low, $\sim 1-2 \mathrm{~J} / \mathrm{cm} 2$ at $8.3 \AA$. The sensitivity increases at longer $x$-ray wavelengths. Features of $<0.02 \mu \mathrm{m}$ can be fabricated.

\section{Develop}

PMMA and copolymer resists are compatible with immersion $\left(21^{\circ} \mathrm{C}\right)$, spray puddle, and spray process modes. Process variables such as soft bake, exposure conditions, choice of resist and developer should be optimized to achieve desired results. For more process details see the PMMA and Copolymer DEVELOPER data sheet. Table 1 lists commonly used developers and their recommended usage.

\section{NANO $^{\text {TM }}$ PMMA AND COPOLYMER DEVELOPERS ARE AVAILABLE IN THE FOLLOWING BLENDS}

\section{PRODUCT COMPOSITION RESOLUTION SENSITIVITY/ \\ THROUGHPUT}

\begin{tabular}{|cccc} 
M/l 1:1 & 1:1 MIBK to IPA & high & high \\
M/1 1:2 & 1:2 MIBK to IPA & higher & medium \\
M/1 1:3 & 1:3 MIBK to IPA & very high & low \\
MIBK & MIBK & low & high \\
& & & \\
\hline
\end{tabular}

\section{Table 1}

\section{Rinse and Dry}

To terminate the develop process and prevent scumming, PMMA and copolymer should be immersed or sprayed with $1: 3$ or $1: 4$ MIBK:IPA, alcohol or DI water immediately following develop. Substrates are normally spin dried at 3000rpm for 20 seconds or $\mathrm{N}_{2}$ blow dried.
Table 2 outlines helpful guidelines for a develop process.

\section{TYPICAL DEVELOPMENT PROCESS}

\begin{tabular}{|c|c|c|c|}
\hline ACTION & SPRAY** & SPRAY PUDDLE & $\operatorname{IMMERSION}\left(21^{\circ} \mathrm{C}\right)$ \\
\hline Dispense & $500 \mathrm{rpm}$ for $30-45$ secs & 500 rpm for $3-4$ secs & \\
\hline Dispense & & 0 rpm for 2 secs & \\
\hline No Dispense & & 0 rpm for $25-40$ secs & 30 secs \\
\hline Rinse * & $500 \mathrm{rpm}$ for $30-45$ secs & 500 rpm for $3-4$ secs & 30 secs \\
\hline Dry & $500 \mathrm{rpm}$ for 30 secs & 5000 rpm for 30 secs & Nitrogen blow dry \\
\hline \multicolumn{4}{|c|}{ * Recommended Rinse solution is MIBK to IPA 1:3 in order to reduce the possibility of scumming } \\
\hline
\end{tabular}

\section{Table 2}

\section{Postbake/Hardbake (optional)}

To remove residual developer, rinse solvent, and moisture from the resist image.

Hot Plate OR $\quad 100^{\circ} \mathrm{C}$ for $60-90 \mathrm{sec}$

Convection Oven $\quad 95^{\circ} \mathrm{C}$ for $30 \mathrm{~min}$

Note: PMMA images will round/flow above $125^{\circ} \mathrm{C}$.

\section{Remove}

Wet: Remover PG or ACRYL STRIP

Bath: time as required, ambient

Spray: time as required, $500-1000 \mathrm{rpm}$

Dry: $\quad$ plasma $\mathrm{O}_{2}$

PMMA and copolymer resists can be removed by using MCC's Remover PG or standard cleanroom solvents, such as acetone, photoresist thinner, or positive photoresist removers.

Resists that have seen higher processing temperatures and/or hostile processes that have toughened the polymer will require ACRYL STRIP or a more aggressive removal process. This can include Remover PG at elevated temperature followed by cleaner baths to assure adequate material removal.

See appropriate product data sheet for specific process recommendations and safety precautions.

For additional questions or technical assistance please contact Technical Services. 


\section{SPIN SPEED CURVES FOR PMMA AND COPOLYMER RESISTS}

The spin speed versus film thickness curves displayed in figures 1-11 provide approximate information required to select the appropriate PMMA or copolymer resist and spin conditions needed to obtain the desired film thickness. Actual results will vary and are equipment, environment, process and application specific. Additional resist dilutions to obtain other film thicknesses are available upon request.

495PMMA C Resists

Solids: $2 \%-6 \%$ in Chlorobenzene

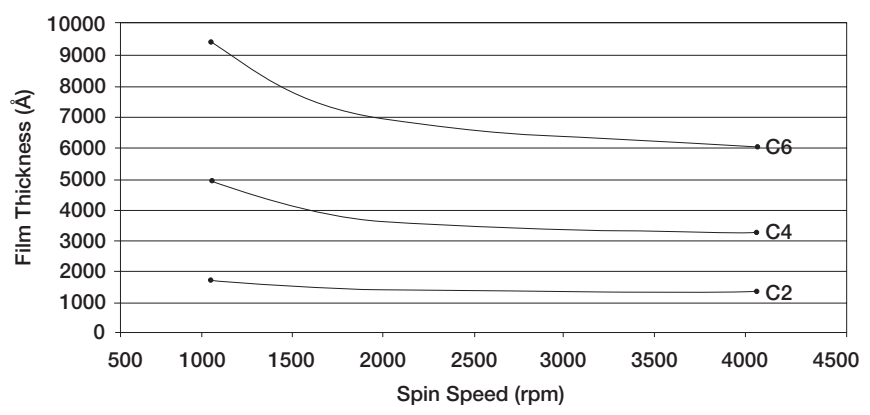

Figure 1

495PMMA C Resists

Solids: $\mathbf{8 \%}-\mathbf{9 \%}$ in Chlorobenzene

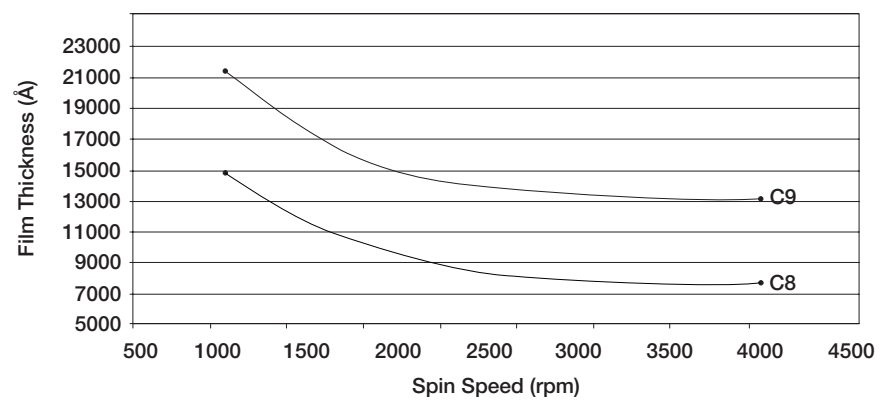

Figure 2
495PMMA A Resists

Solids: $2 \%-6 \%$ in Anisole

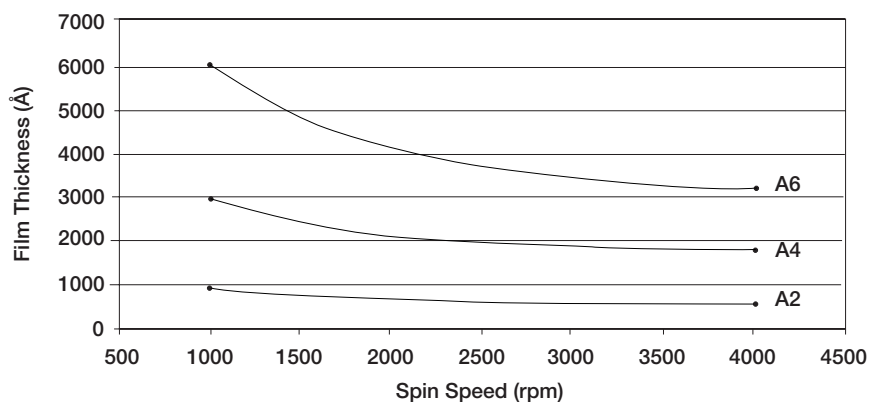

Figure 3

495PMMA A Resists

Solids: $8 \%-11 \%$ in Anisole

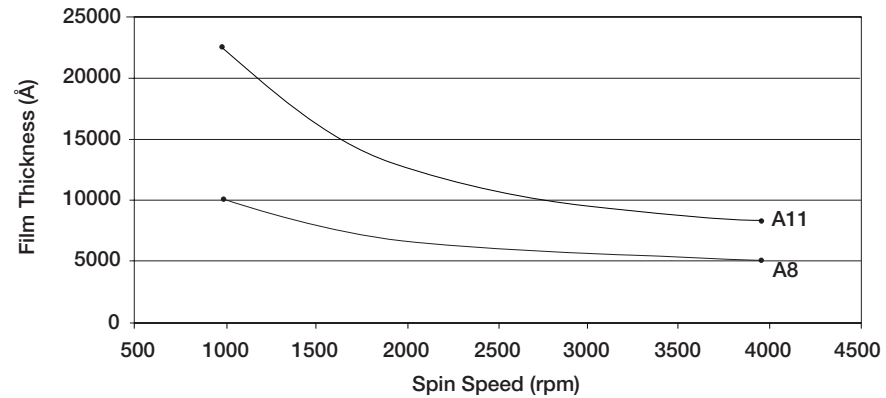

Figure 4

\section{Copolymer Resists}

Solids: $6 \%-11 \%$ in Ethyl Lactate

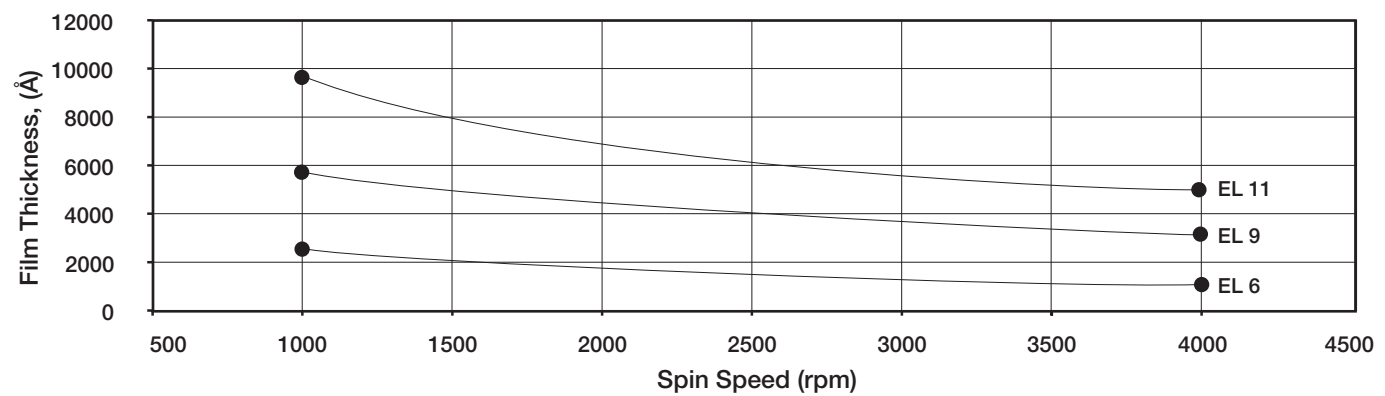

Figure 9 
950PMMA C Resists

Solids: $\mathbf{9 \%}-\mathbf{1 0 \%}$ in Chlorobenzene

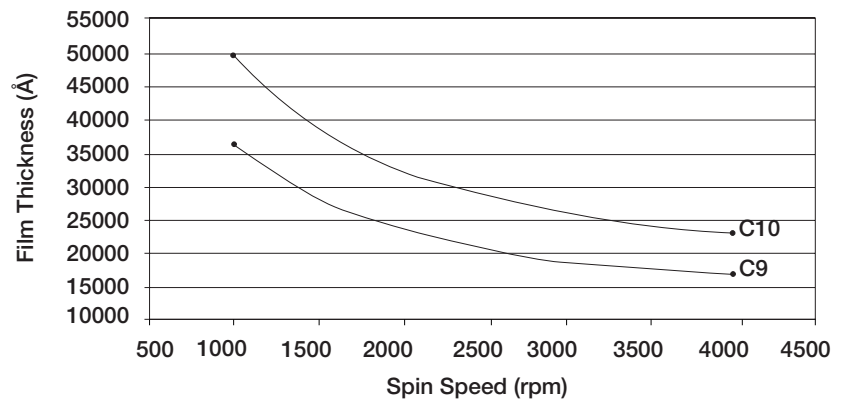

Figure 5

950PMMA C Resists

Solids: $\mathbf{2 \%}-\mathbf{7 \%}$ in Chlorobenzene

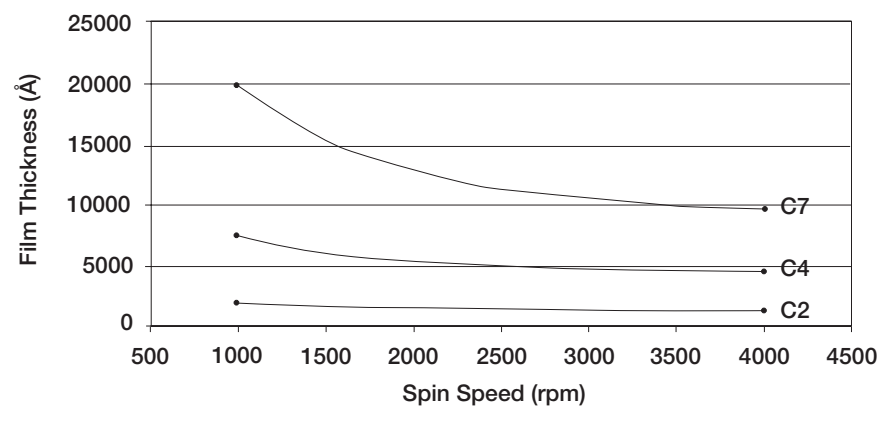

Figure 6

Optical Properties

495 and 950 PMMA Resists

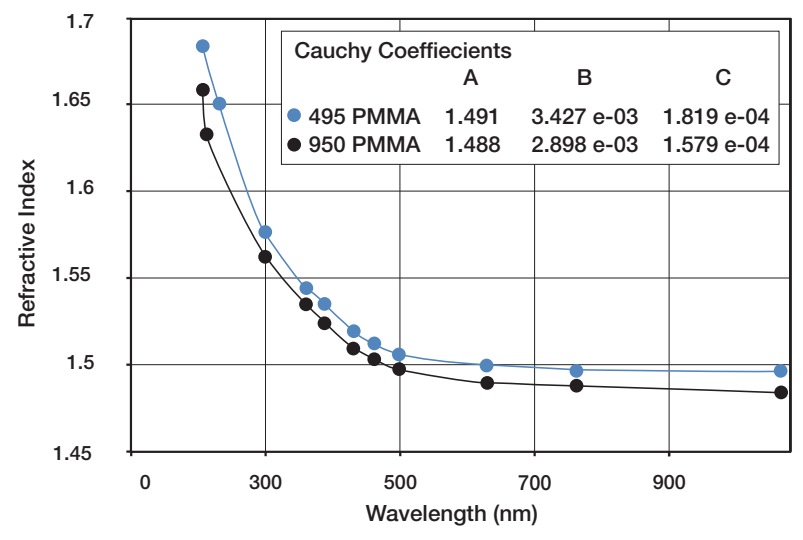

Figure 10
950PMMA A Resists

Solids: $9 \%-11 \%$ in Anisole

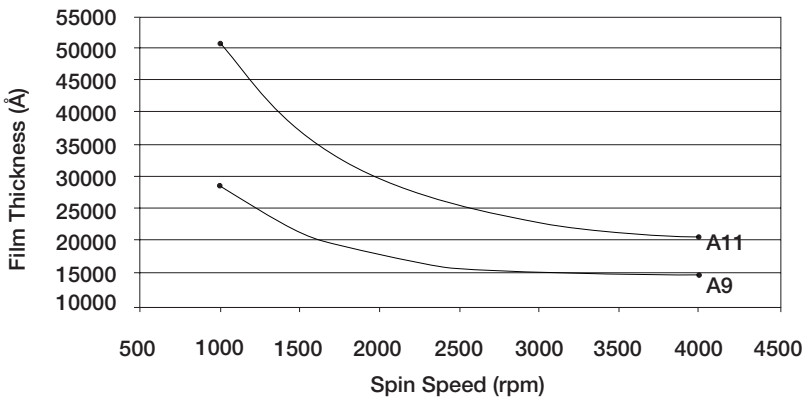

Figure 7

950PMMA A Resists

Solids: $2 \%$ - 7\% in Anisole

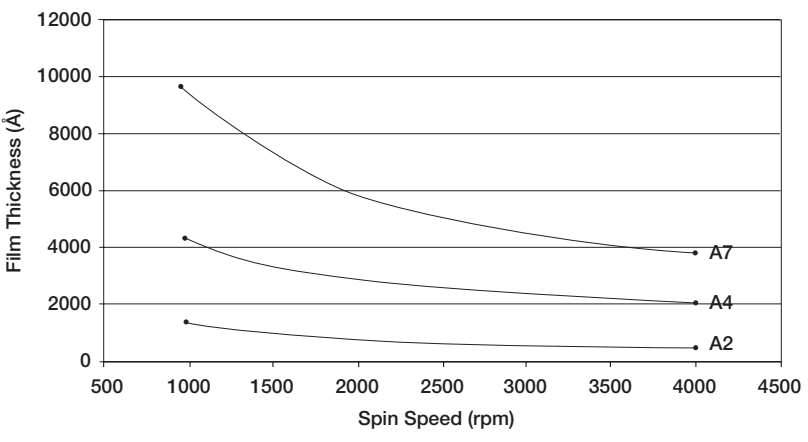

Figure 8

\section{Optical Properties}

Copolymer Resists

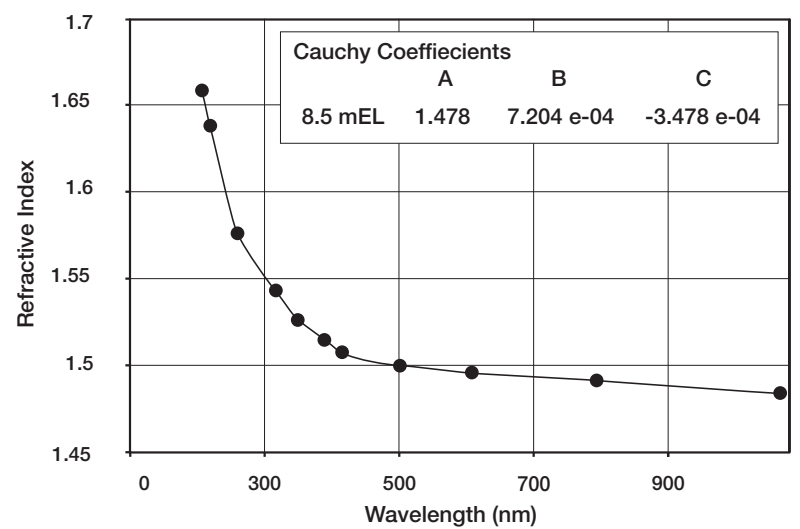

Figure 11 


\section{PMMA resists for T-gate and other imaging processes}

PMMA is a high resolution positive tone resist for e-beam, deep UV (200-250nm) and X-ray lithographic processes. Although PMMA may be used in a single layer resist process, it is most commonly used in multi-layer processes such as in the fabrication of mushroom or T-gates. Images are formed through the photo scission of the polymer backbone and subsequent development process, which removes the exposed, lower molecular weight resist. Multi-layer, shaped resist profiles are realized and influenced through the careful choice of PMMA molecular weight, film thickness and other process set points.

In a typical bi-layer process, a combination of bottom and top layer resists are selected such that a large difference in dissolution rates of the layers at the developer step exists, leading to the desired resist sidewall profile. This contrast may be further influenced with a variety of process strategies. Generally, dissolution rate increases as molecular weight decreases. However, soft bake conditions, which affect residual solvent level and subsequent development rates will influence the bi-layer resist profile as will the exposure conditions.

Please refer to our web site, www.microchem.com for applications notes concerning non-imaging PMMA processes such as wafer thinning, bonding and sacrificial layers.
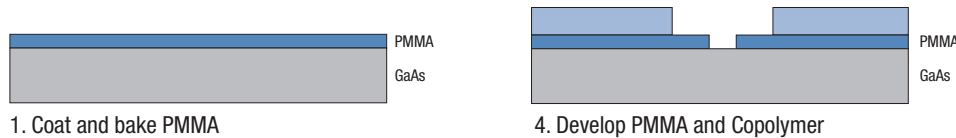

4. Develop PMMA and Copolymer

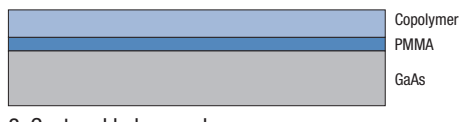

2. Coat and bake copolymer
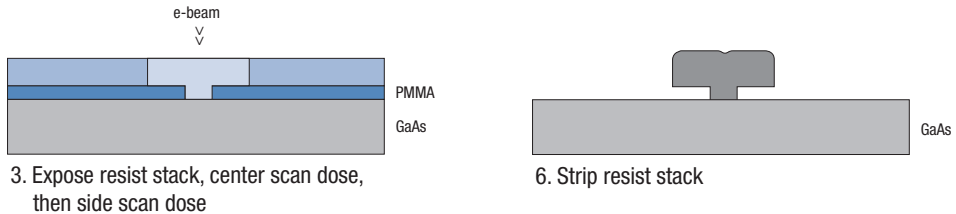

\section{Tri-Layer Process}

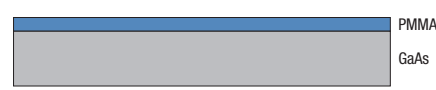

1. Coat and bake high MW PMMA

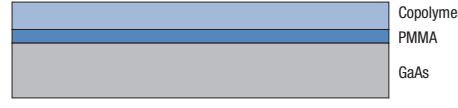

2. Coat and bake copolymer

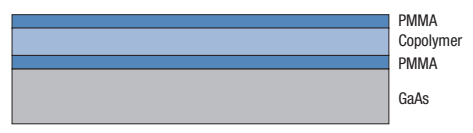

3. Coat and bake low MW PMMA

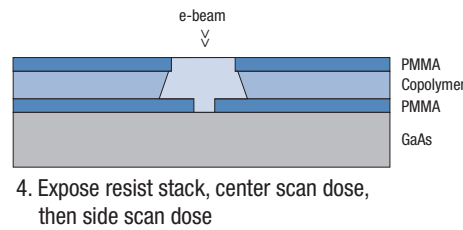

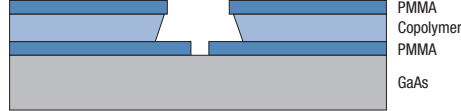

5. Develop tri-layer stack

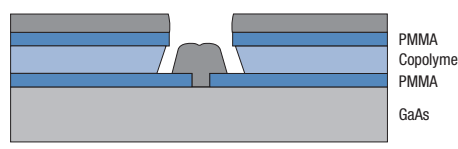

6. Deposition

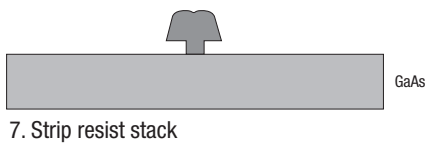




\section{OTHER RESIST AND ANCILLORY PRODUCTS FROM MICROCHEM}

PMGI and LOR for lift-off processing

SU-8 resists for MEMS and ultra thick resist processing

Remover PG resist stripper

EBR PG edge bead remover

Acryl Strip resist stripper for PMMA

\section{NOTES}




\section{HANDLING NANO PMMA}

\& COPOLYMER SERIES RESISTS (in Anisole or Chlorobenzene)

Use precautions in handling flammable PMMA solutions. Avoid contact with eyes, skin, and clothing. Use with adequate ventilation. Avoid breathing fumes. Wear chemical-resistant eye protection, chemical gloves (PVA for chlorobenzene solutions) and protective clothing when handling NANO PMMA \& Copolymer Series Resist products. NANO PMMA \& Copolymer Series Resists cause irritation in case of contact with eyes, skin, and mucous membranes. In case of eye contact, flush with water for 15 minutes and call a physician immediately. Review the current MSDS (Material Safety Data Sheet) before using.

\section{MATERIAL AND EQUIPMENT COMPATIBILITY}

NANO PMMA \& Copolymer Resists are compatible with glass, ceramic, unfilled polyethylene, high-density polyethylene, polytetrafluoroethylene, stainless steel, and equivalent materials.

Chlorobenzene is a powerful solvent and will attack various elastomers such as BUNA N, EPDM, HYPALON, and NEOPRENE. It will also attack PVC, CPVC and polyester. VITON $A$ is recommended for both 0 -rings and tubing.

\section{PROCESSING ENVIRONMENT}

For optimum results, use NANO PMMA \& Copolymer Series Resists in a controlled environment. $20-25^{\circ} \pm 1^{\circ} \mathrm{C}\left(68-77^{\circ} \mathrm{F}\right)$ is suggested.

\section{STORAGE}

Store upright in original containers in a dry area above $50^{\circ} \mathrm{F}$. Do not refrigerate. Keep away from sources of ignition, light, heat, oxidants, acids, and reducers. Shelf life is 13 months from date of manufacture.

\section{DISPOSAL}

Each locality, state, and county has unique regulations regarding the disposal of organic solvents such as NANO PMMA Series Resists. It is the responsibility of the customer to dispose of NANO PMMA Series Resists in compliance with all applicable codes and regulations. See MSDS for additional information.

\section{MICRO CHEM}

1254 CHESTNUT STREET

NEWTON, MA 02464

PHONE: 617.965 .5511

FAX: 617.965 .5818

EMAIL: mcc@microchem.com

W W W . M I C R O C H E M . C OM
The information regarding these products is based on our testing to date, which we believe to be reliable, but accuracy or completeness is not guaranteed. We make no guarantee or warranty, expressed or implied, regarding the information, use, handling, storage, or possession of these products, or the application of any process described herein or the results desired, since the use and handling of these products are beyond our control. 